\title{
Q L
}

545.2

F94

1922

ENT 

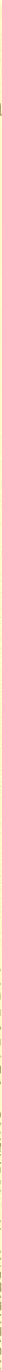
LIBRARIES SMITHSONIAN
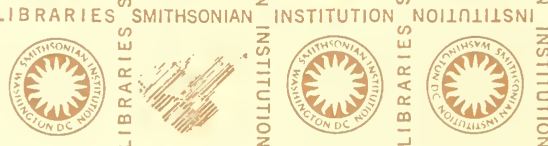



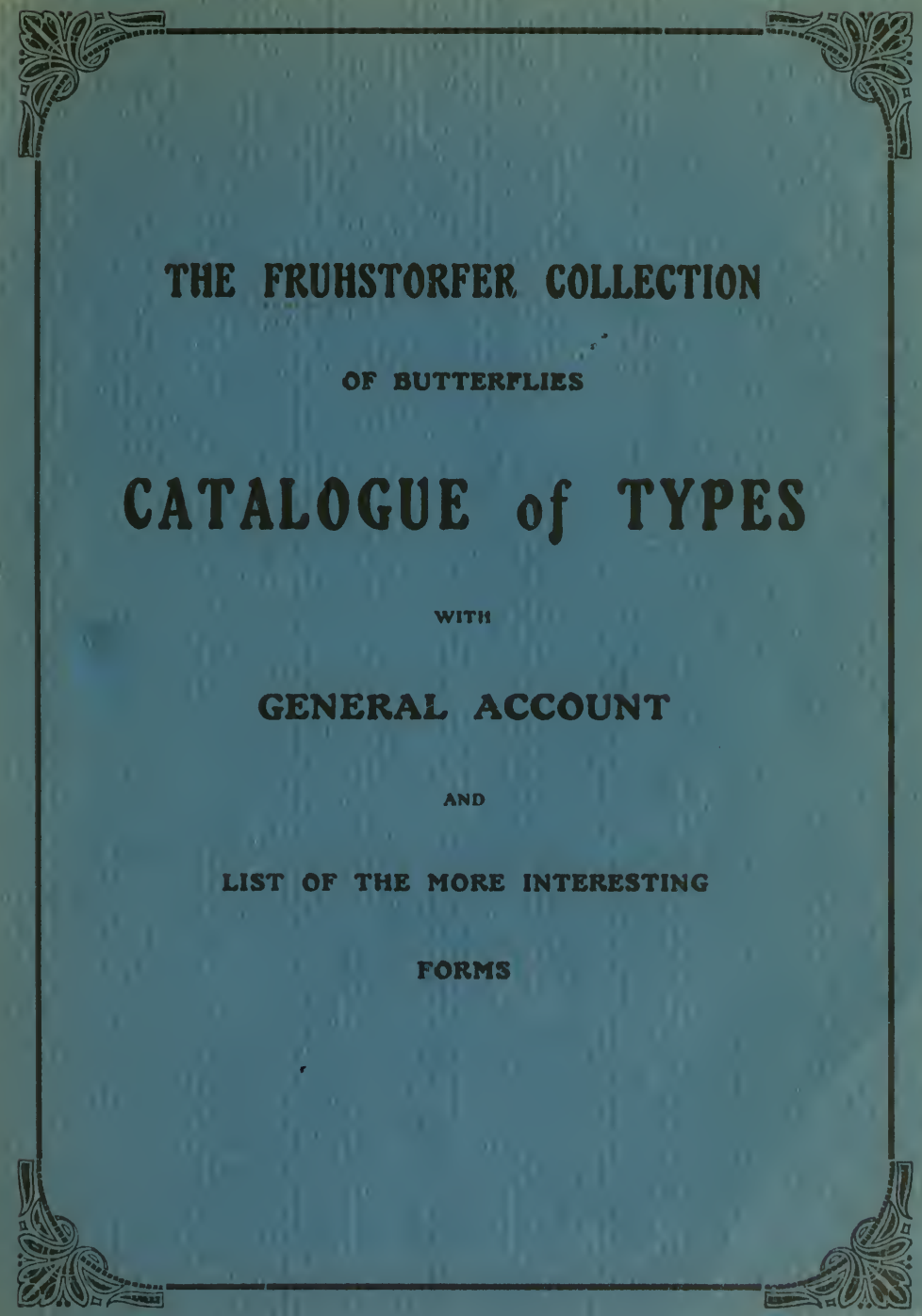


ando

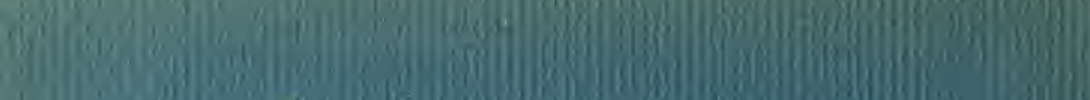

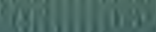

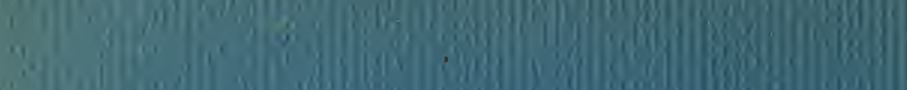
acting mons (10.6) (5) (ing 15:-3ing "

(c)

$(6)\left(x_{0}\right)$

3.

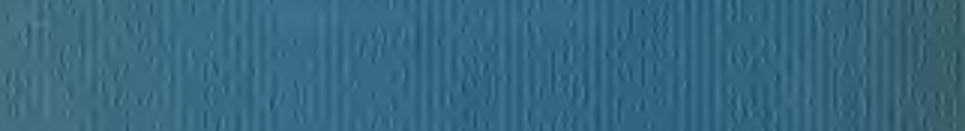

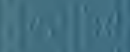

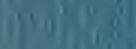
(x) is

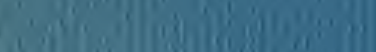

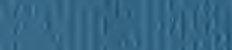

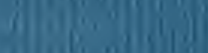


HANS FRUHSTORFER

BY

$\mathrm{D}^{R}$ L. MARTIN

Published in the "Iris" of November 3oth, I922.

高紫

as supplement or added gant

To Doutsehe Entomologisehe Zaitschript "Irig" herauggel en vom Frytomdog 'sehen Verein Jius Dresden

vol, xxxui, Heed $3 / 4$ Noviso, 



\section{HANS FRUHSTORFER}

It would seem an unpardonable omission not to devote a few words of appreciation and regret to the memory of the late Hans Fruhstorfer in the columns of the "Iris" in which his name was so often to be found and to which he was one of the most prolific and most valuable contributors:

I feel it is incumbent upon me to fill this historical gap in view of the close scientific association and the warm friendship which united us for over thirty years. I must confess, however, that I have great difficulty in finding adequate words wherewith to do justice to Fruhstorfer's entomological eminence, his scientific achievements and the originality of his genius.

Want of time compels me unfortunately to give nothing but the briefest outline of the most important facts of his life - had I been able to follow the dictates of my heart, I should have written a detailed biography.

After having undergone several operations Fruhstorfer succumbed in the night of April 7 th to 8 th to that fell disease, intestinal cancer. During one of his strenuous expeditions in the tropics (in Igor, when he was exploring the interior of Siam along the Mekong) he contracted dysentry of a malignant type from which he never entirely recovered and which no doubt prepared the ground for the dread germs of cancer of which, alas, so little as yet is known.

He was laid to rest on April IIth. Only very few of his friends were able to accompany him to his last resting place, since he had kept the nature of his illness and its inevitably tragic issue a secret, not only from his friends, but even from his nearest and dearest relations in Switzerland: partly, no doubt, because he, hitherto so active, so vigorous and energetic, could not bear the thought of being pitied in his stricken condition; and partly, perhaps, because it is the fate of those whom true genius sets on a pinnacle to seek even in death the solitude they loved in life.

The word "genius" really describes Fruhstorfer. His was a purely. entomological and systematic genius, the like of which we have never met before and are not likely to meet again. 
Less recent authors, such as Cramer, Hewitson, Felder and others have also described hundreds of new butterflies, but they were actuated by purely aesthetic or sporting considerations and their works lack the scientific basis and cohesion which are so striking in Fruhstorfer's works. $\mathrm{D}^{\mathrm{r}} \mathrm{O}$. Staudinger, who worked in the same field, was his predecessor and much admired model, and de Nicéville, Rothschild and $\mathrm{D}^{\mathrm{r}}$ Jordan were his collaborators, He was born on March 7 th, r866. In I888, at the age of 22, he started on his first collecting expedition, to Brazil. During his two years' stay in South America he acquired an intimate knowledge of the fauna of South America - which is of special importance for all students of tropical insects - and was subsequently able to contribute to Seitz's great work important articles on several little-known families of butterflies peculiar to those regions. These contributions compare very favourably with those of the other authors in the same field; they seemed to me like oases in a sandy waste. From the financial point of view also Fruhstorfer's South American journey must have been successful, for as early as 1890 , after a prolonged stay in Ceylon in search of new specimens, he had acquired complete financial independence and, free from all impediments, was working in Java where he devoted more than three years to entomological research in every part of that island paradise. He declared that his stay at Java had marked the beginning of his success as a collector" - a career which, at the outset, he had found full of difficulty - and that "the years spent in that island had been the happiest of his life and the most active, the most memorable of his youth". Those who know Java, that earthly paradise, will sympathise with him. Fruhstorfer's knowledge of the fauna of Java has never been excelled and even the Dutch who, after all, have possessed and exploited the island for more than three hundred years have never produced anyone who could be compared with him. He was the first to recognize the remarkable difference which exists between the fauna of the western part of the island where the rainfall is abundant and that of the dry eastern part, and during those years he discovered a large number of new species and varieties.

The collection he formed at Java remained his most cherished possession. It was kept separate, and was never amalgamated with the rest of his Indo-Malayan collection. There was something touching in his love for all the creatures of that island which, compared with those of the same group in the other islands, he always declared to be the most beautiful, the most finely shaped and the most gorgeously coloured of all.

His enthusiasm may have been justified in many cases, but it is 
difficult for anyone not possessing his intimate knowledge of the Malay Archipelago to judge or to express an opinion.

The beginning of our lifelong friendship also dates from that period i.e. I89I, when we exchanged material from Java and Sumatra.

He made a critical study of the material I had collected in Sumatra and found several varieties to be distinct and peculiar to that region. Thus it came about that many Sumatra butterflies, caught and discovered by me, bear his name. Although I ceded to him a new and hitherto undescribed papilio (iswaroides) I feel no jealousy now, for in those days it would have been impossible for me, a doctor with an extensive practice in Sumatra and without leisure or the necessary books, to devote myself to these studies. Moreover, in those days I still was a convinced opponent of the practice of giving geographical names to certain varieties which in later years, following in Fruhstorfer's footsteps, was to afford me such infinite pleasure.

It was at Java that he discovered the only known specimen of gauroides, a species of Elymnias which closely mimics an Ideopsis. No other specimen has ever been caught, either by him or by anybody else. It is possible that this specimen belonged to a species which has since become extinct and that Fruhstorfer caught one of the last existing specimens; or perhaps this animal may eventually be found to be an extremely rare dimorphous female of one of the known species.

During the years 1895 and $I 896$ Fruhstorfer undertook a further journey to the Malay Archipelago for collecting purposes. He visited Celebes and the smaller Sunda Islands, Bali and Lombok, where he collected a vast amount of material; this expedition, also, proved very profitable from a pecuniary point of view. The very peculiar butterflies of Celebes especially were almost unobtainable before Fruhstorfer undertook this journey and many were only known from illustrations. Only Messrs. Staudinger still possessed some specimens left over from the late $D^{\text {r }}$ Platen's collection.

On Celebes also he immediately recognized the marked difference existing between the varieties in the north and those in the south of the island; like Wallace, he collected both at Macassar and at Menado.

He lost no time in publishing descriptions of his valuable discoveries and in classifying and naming them. At Lombok he had the good fortune to meet that "Prince of collectors", the American, Mr. Doherty and to have an opportunarity of working with him for a weck.

The peculiarity of the fauna of the smaller Sunda Islands - a sharply defined region which Fruhstorfer called Mikromalayana in contrast to the greater Sunda Islands (Makromalayana) - had up to that time not 
been sufficiently known or appreciated. Fruhstorfer drew attention tc it and demonstrated it in his work on the Lombok butterflies, thus contributing a valuable quota to the common fund of knowledge of entomologists.

After his return from this journey he published his first systematic works which formed the basis of his subsequent achievements and, in particular, of Seitz's great work.

Most collectors in tropical countries either pay with their lives for their fearlessness and enterprise or, upon their return to Europe, areswindled by the dealers and robbed of the fruits of their labours. Fruhstorfer forms a brilliant exception, for he knew how to retain for himself the profit derived from his toilsome journeys.

Europe did not, however, long satisfy his enterprising spirit.

In June I 899 he started upon another journey which lasted the greater part of three years and in the course of which he visited America, the South Seas, Japan, China, Tonkin, Annam and Siam Everywhere he collected with dauntless energy, generally in regions which were partially or wholly unexplored. He accumulated an immense amount of material: lepidoptera, coleoptera and conchyliae, enough to keep research workers occupied for many years to come.

In Siam he unfortunately contracted dysentry and this illness put an end to his bold and far-reaching plans. He was forced to return sooner than he had intended, travelling by Singapore, Rangoon, Darjeeling and the mainland of India. He has described the incidents of this adventurous journey with spirit and humour in his "Tagebuchblätter" ("Pages from my Diary"), a book which is now unfortunately very rare and might be read with enjoyment by the general public as well as by specialists.

The material gathered in this, the last of his scientific travels, was also plentiful and of the greatest interest and value.

Fruhstorfer then settled in. Geneva, on the banks of the Arve, close to the French frontier, where he built himself a picturesque home large enough to house his collections. In the succeeding years he published the results of his researches and of the study of his collections. In addition to numerous descriptions of new species and varieties published in the press, he began to publish his valuable entomological works, monographs on genera of butterflies such as Taenaris, Cethosia, Hebomoia, Elymnias, Mycalesis, Neptis, Athyma and many others. These monographs were subsequently used as a basis for the text of Seitz's work. As a collaborator in this standard work he certainly ranks, as the greatest authority next to Professeur Aurivillius and Dr. Jordan. 
All those who, before the publication of his works, have had to identify and classify exotic butterflies will fully appreciate the enormous advance made by Fruhstorfer in this field.

None of the species he has studied will need to be dealt with again for many years to come. His nuain endeavour was to limit as far as possible the use of the term "species", which is used in far too wide a sense, and to identify and classify known species with reference to all their geographical and seasonal variations.

In the text of Seitz's great standard work he solved this problem wherever possible, in the most brilliant manner. It is true that a few mistakes and inaccuracies have crept into this text, but Fruhstorfer can hardly be made responsible for these. They are mainly due to the speed with which the editor - urged, perhaps, by a sad presentiment was endeavouring to get the work done.

Such part of Seitz's work as has been successfully completed could never have been produced in these days, - less than ever now that Fruhstorfer has gone.

So far as I know, he was also the first to make successful use, in determining species, of the anatomical study of the male genital organs of butterflies.

Following the example of his eminent predecessor, Dr O. Staudingerby whom be was influenced more, perhaps, than he was himself aware, but whom he excelled in acumen and penetration -- he subsequently sent out collectors on his own account to tropical countries. Thus he sent collectors to the distant island of Engano in western Sumatra, sent Sauter to Formosa and Dr. Werner to New-Guinea.

He identified and described a large number of new varieties brought from Formosa and thus went further even than the Japanese who want to identify the Indo-Malayan fauna of that island with their own purely palaearctic fauna.

From his retreat in Geneva Fruhstorfer also turned his attention to the Palaearctae and later to the Orthopterae, thus abandoning for a time the study of his beloved butterflies. Even in that field of research he did pioneer's work and acquired in a short time an astonding amount of knowledge.

Alas, he did not live to see the publication of his great work on the Orthopterae of Switzerland which appeared shortly after his death.

During the last year of his life, however, he contritely returned to his beloved Lepidoptera and we owe to this renewed interest some exceedingly valuable chapters on Malayan Lycaenidae in Seitz's work. They represent an immense advance on the work of his predecessors. 
This very brief account will serve to show what an immense amount of work Frushtorfer achieved in the course of his life: that his work was pioneer's work in every sense all those who have studied tropical butterflies both before and after his time will gratefully acknowledge.

It is now possible, just by glancing as Seitz's work, immediately to identify any exotic butterfly whose habitat is known - provided that the species in question has been dealt with by Fruhstorfer.

In forming an estimate of Fruhstorfer's achievements the fact must be borne in mind that he was entirely self-taught, that be acquired without outside assistance a certain knowledge of the classical languages and learnt to speak four modern languages fluently. In the latter years of his life he even acquired a very considerable knowledge of botany, to which his "Tessiner Wanderbilder" (Wanderings in the Ticino) - a book which is well worth reading - testify.

In his work he had, of course, to contend with many old-established mistakes and prejudices, to sweep away many deeprooted notions and thereby frequently aroused embittered opposition. Like all really great and original minds he did not everywhere meet with the apprecia. tion he deserved and was subjected to narrowminded criticism in many quarters.

In this connection I need only recall the very varied judgments passed on Richard Wagner in his life-time. Such men must be forgiven any slight peculiarities and imperfections which may mar the general tenor of their lives, in view of the magnitude and value of their achievements.

Those who, like myself, have enjoyed the privilege of spending long evenings in stimulating scientific converse with that accomplished and always courteous man will ever do justice to the memory of his exceptional qualities. But he himself has left the best and most enduring monument - aera perennius - to his memory, namely his works. In him we have lost far and away the best connoisseur of tropical butterflies and I know of no man who would have the genius, the necessary energy or the means to replace him.

Dr L. MARTIN

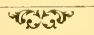




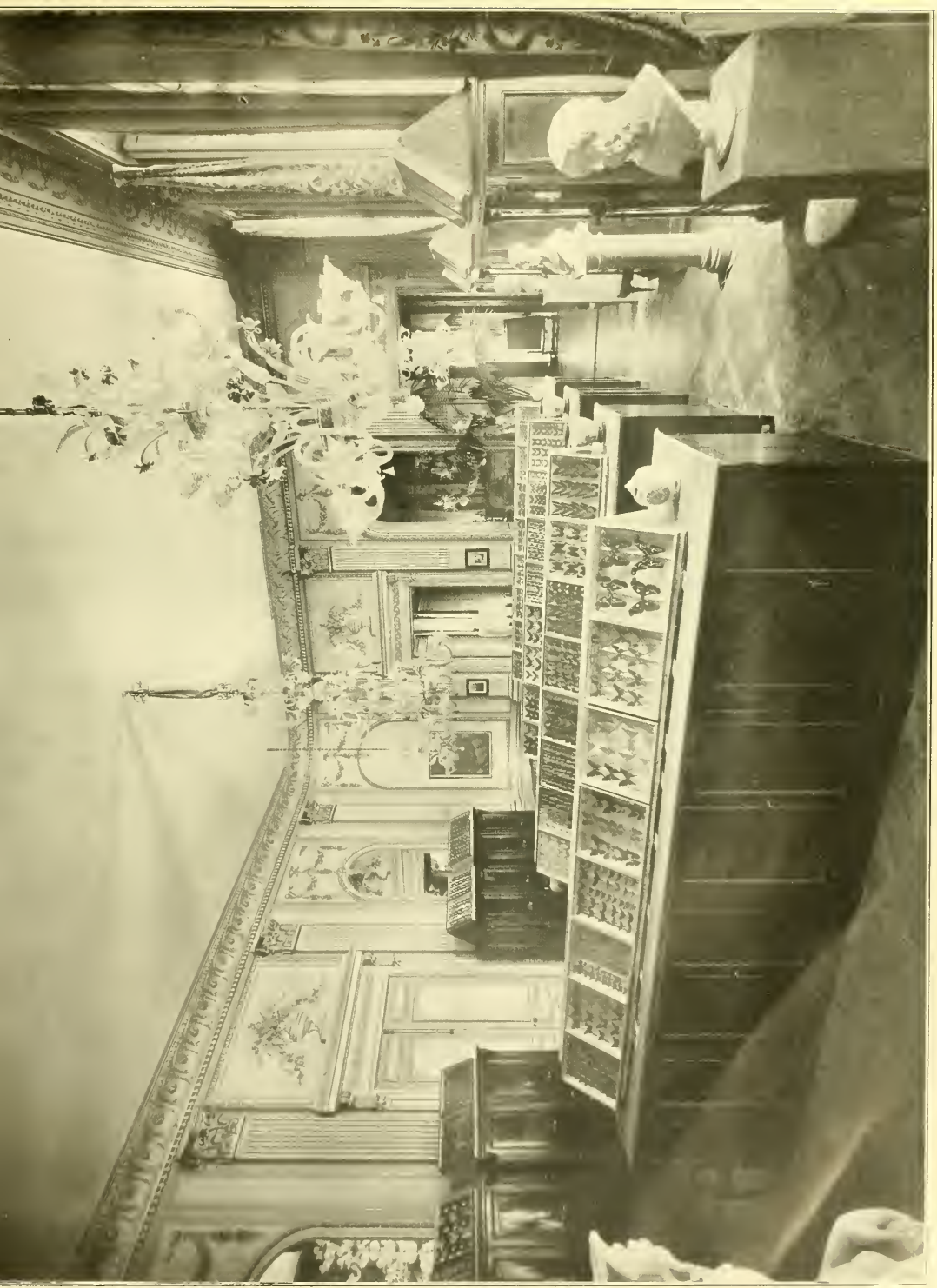





\section{FRUHSTORFER COLLECTION}

FIRST LIST OF TYPES

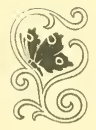





\section{PAPILIONIDAE}

Troides paradisea ab. auriflua priamus $f$. divina poseidon $f$. Nivalis priamus Eudamidas Aeacus Bazilanica Aeacus f. preacox euthycrates agittatus nereides mosychrus amphrysus Euthydemus oblonomacuratus ab. carorus helena hanno Amphrysus vistara

Papilio Noctis solokanus

Polydorus sabinus kajeranus polydorus ceramites naissus arbosignatus asinius lasoarus voluptitina aphnitis

hector heloicus polyphontes seganus ouijovus aipytes extensus

philoxenus hostilius aristolochiae plopus poseidippus antiphulus kameilos Lombokensis $f$ askra balinus mamilus

slateri ab. jantivius agestor senchalus agestorides matsumurae

epycides curdatus euchenor scribonus

lasos eutrobius - $f$, marga hippotus euchenides

gigon mangelinus

1" gargilius
New Guinea

Amboina

Waigeu

Cerm "N N. G.

Bazilam

Siam

Tonkin

Lombok

Bawean

N. Borneo

N. E. Sumatra

New Guinea

Goram

Batu

Sumatra

$\mathrm{Obi}$

Buru

Dutch N. G.

Fergusson

Milne Bay

Waigeu

Germ. "N. G.

New Guinea

Goodenough

Ceylon

Batjan

Talaut

Sula

Saleyer

Lang-Bian

Bawean

Razilam

Jolo

Nias

Lombok

Bali

Engano

Assam

Sikkim

Lon-Tse-Kiang

Formosa

Upperburma (female)? (male)

Waigeu

Milne Bay

Obi

New Guinea

Fergusson

Sula

Bangkei 
Papilionidae (Continued)

Papilio demolion energetes

demolion messius

n pictus

eriphonius annamitius

xuthus tyrtanus

neoxuthus $f$. xuthris

xuthus neoxuthus

n practius

xuthulimus $f$. honadensis

tyrtamus $f$. selleis

xuthus kosinga

machaon phrygius

arcinous bradannus

philoxenus termessus

pelytes $f$. rubida

neomeoanides

f. cyroides

vigellius

messius

passikrate

polyctes

nicomachus

alpheios

passienus

polycritos

falcidius

ambrax decebaris

$$
\text { f. lutosa }
$$

akanes

marzaias

musaliki

ornemus $f$. leucotaenia

$$
\begin{aligned}
& \text { f. dolorosa } \\
& \text { f. colasa } \\
& \text { arachosius } \\
& \text { arachosius f. amisia }
\end{aligned}
$$

aegeus aegates

olmienuous

bridgei nobiliol

helenus seminus

orosinus

aulus

nechelenus

daksha Hamps.

apadantis

fortunius

iswaroides
Nias

Loinbok

Sumba

Annam

Hosima (male)

Nagasaki (female)

Siaou-Lou

Ta-Tsien-Lou

Sapporo

Japan

Nagasaki

Formosa

Smyrne

Ishikagi

Formosa

Malabar

Malacca (male)

Syngapore (female)

Sikkim

Bawean

Lombok

Formosa

Okinawa

Buru

N. Celebes

E. Celebes

Sula Besi

Bangkei

New Guinea

Arfak

Fergusson

Milne Bay

N. Irland

Germ. "New Guinea

Germ. "New Guinea

Waigeu

Waigeu

Waigeu

Milne Bay

Fergusson

Solomons

Japan

Oshima

Hainan

N. Borneo

Nilgiris

Malacca

Formosa

N. E. Sumatra 
Papilionidae (Continued)

Papilio chaon durius

duenarius

dispensator

f. lucacantha

rileyi

nephelus tellenus

fuscus madamus

1. lapathus

" metagenes

") ebibonnus

pyrgoteres

vitylus

offakus

albinus lamponius

capaneus yorkianus

beccari $f$. anna

rumanzovius cirus

" spinturinae

memnon perlucidus supcrathratus

protënor euanthes

euprotenor

sulpitius

demetrius lenecinnus sitarkaes

liukiuensis

rhetenor $f$. arbolineata

rhetenor pratonius

aurificatus

publilius

amoena

rupruntus

bianor okinawensis

$$
\text { gladiator }
$$

paris majestatis

it f. gemmifera

f. decorosa

aurigennus

karna suriya

thrasymedes

polyctor titus

arcturus arcturulus

palinurus nikagoras

solinus

tubera

ulysses marius
Formosa

Lower Burma

Tonkin

Darjeeling

S. Szuchuan (China)

Batu

Buru

Batjan

Binongka (male

Kalidupa (female)

Florida 1.

St. Aignan

Yule 1.

Waigeu

Germ. "New Guinea

N. Australia

Arfak

Mindoro

Talaut

Lombok

Flores

Hainan

Sikkim

Tonkin

Nagasaki

Okinawa

Ishigaki

Sikkim

Ichang

Sikkim

Lower Burma

Formosa

Tsekou

?

Tonkin

Tonkin

Tien-Tsuen (male)

Mou-Pin (female)

Sikkim

Siam

Sintang (Borneo)

Formosa

Tonkin

Tsze-Tchuan

Sumatra

N. Borneo

S. E. Borneo

Mioko

19

$q$
0
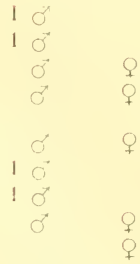

1

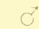

1

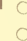

o

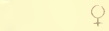




\section{Papilionidae (Continued)}

Papilio ulysses nigerrinus Ribbe.

$$
\text { telephanes }
$$

pericles olympiodorus

$$
\text { hekaton }
$$

hermogenes

peranthus baweanus

agetes tenuilineatus

kinabaluensis Tetens. iponus

aristius aristinus

nomius pernomius

rhesus rhesulus

antiphates antiphonus

sarpedon tenus

ab. aureiterus
halesus
coelius
corycus f. croscospilos
nipponus
f. sarpedonides
morius
ruckakius
pagus
colus
rufofervidus
meles
messogis
tenuus

eury['ylus sulanus

sallastinus
gabinius
sanius
crispus
georgius
lutorius

Papilio eurypylus prisius

$$
\begin{aligned}
& \text { daton } \\
& \text { tagalicus } \\
& \text { jupa }
\end{aligned}
$$

deson axion $f$. aurifer

$$
\text { axion f. praestabilus }
$$

actor

nanus

perillus

jason postianus

$$
\text { sankapurus }
$$

arycles arycreoides

sphynx
Solomons

Kirwina

Babber

Letti

Selaru

Bawean

Tenasserim

? Kina Balu

Malacca

Kalao

Siam

S. Celebes

Nias

"Germ. "New Guinea

Ceram

Buru

Sula

Waigeu

Japan

Oshima

Ishigaki

N. Borneo

Bazilan

Palawan

Nias

Tonkin

Key

Dutch New Guinea

Sula

Sumba

Sumbawa

Tanah Djampea

Babber

Obi

Batjan

Waigeu

Java

Bazilan

Hainan

Siam

Tonkin

Hainan

Annam

Ishigaki

Formosa

Bawean

Siam (male)

Cochinchina (female)

Indo-China (male)
10 o

10

18

10

$0^{x}$

10

10

o

o

8

8

$\pi$

o

0

0

o

0

0

o

б

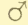

o

0

o

10

o

$\sigma^{x}$

$0^{x}$

o

$\sigma^{x}$

ఠ

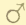

10

10

0

0

0

$0^{x}$

0

o

17

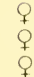




\section{Papilionidea (Continued)}

Papilio arycles inertus

$$
\begin{aligned}
& \text { " } \begin{array}{l}
\text { perinthus } \\
\text { evenus }
\end{array} \\
& \text { evemon alpociliatus } \\
& \text { doson eleius } \\
& \text { " vuloso } \\
& \text { " appuletus } \\
& \text { autronicus } \\
& \text { "gyndes } \\
& \text { pathycles tereus }
\end{aligned}
$$

manlius

agamemnon menides

agamemnon meton

pedius

perecopus

atreus

aelius

atropictus

rufoplenus

mynion

kineas

appius

cloanthus cloanthulus

megarus mendicus megapenthes sagittiger

martinus

delesserti sacerdotalis

xenocles $f$. xenochates

$$
\text { " keplisos }
$$

" lindos

macareus perakensis

Leptocircus meges pesorinus

$$
\begin{array}{cl}
\multicolumn{2}{c}{\text { niasicus }} \\
\text { " pallidis } \\
\text { " annamitica } \\
\text { curius magisthalis } \\
\text { "libelluloides }
\end{array}
$$

Eurycus cressida insularus

Thais polyxena thesto

cerisyi martini

Armandia lidderdali spinosa Stich.
? Singapore

10

Palawan

Palembang

Tonkin

Malabar

E. Java

Sumatra

Natuna

Palawan

Hainan

Palawan

Ceylan

Lombok

Dammer

Babber

Kiriwina (male)

Fergusson (female)

Bawean

Engano

Nias

Australia

Obi

Batjan

Sikkim

Siam

Annam

N. Borneo

Sumatra

W. Sumatra

Darjeeling

Tonkin

Siam

Malacca

Palawan

Nias

Tonkin

Annam

Yunnan

Nias

Wetter (male)

Letti (female)

Saratow

Rhodos

Tsze-Chuan 


\section{PAPILIO (Americain)}

nephalion astanabel polystictus galenus philolaus ab. felicis thoascharmadus thoas astaboras androgeos bagous flaminius lycophron drepanon zagoeus chrysoxanthis cleotas erotuanus anchisiades philastrius hectorides loysirte agoldis

teiesilaus vitellus domitor iphitas evenulata deiioon $f$.

serville boliviana dolicaon draconarius ammon
Rio Grande ?

Honduraz

Bolivia

Rio Grande

Rio Uaupes

Colombea

Rio Grande

Peru

Rio Grande

Trinidad

Rio Grande

Paraguay

Brazil

Brazil

Brazil

Brazil

Bolivia

Rio Uaupes

Brazil

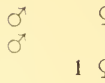

q

19

\%

o

o

8

o

18

$0^{x}$

o

o

$0^{x}$

0

$\sigma^{2}$

$1 \varnothing$

$\sigma^{x}$

10

18

18

\section{PARNASSIUS}

nomion tita.

" theagenes

apollonius gloriosus

dambi

narynus

mnemosyme antitauricus craspedontis

apollo guadarramensis nivatus

hardwicki f. albicans

$$
\text { f. otas }
$$

delphius dolabella

epaphus pernigrus

mercurus cyrnus

acconus

gylippos
Sutschan

Lanchowfu (Kansu)

Alexander Mtns.

Sult. Harret

Turkestan

Antitaurus

Saratow

Castillon

Neuveville

India

Cachemire

Kashgar

Tibet

Aksu

Sikkim

Aksu 


\section{PIERIDAE}

Delias hyparete despoliata Appias hombroni sulanorum vacans $f$. luruda glyncida enaretina

vasava

heppona

purana

f. galbina

papiria

icilia

lutatia

gellia

andrea $f$. aternia

cardena perakana

nephele anfidia

zelima f. sopara

celestina barca

f. lileia
galepsus
f. limia
f. aurifera
galerus

agar

nero ramosa

Appias chelidon

nero hainanensis

$$
\text { foelita }
$$

tiberius

f. sufflaba

baweanicus

pulonys

neronis

sulana

zarinda $f$. aurora

leptis festrada

aemilia

myndus

eurytus

massilia

balinus

varus

lalage f. yami

f. lucila

mutina

argyridina

durvasa $f$. confluens
Sumatra

Sula

Sikkim

Palawan

Malacca (male)

Perak (female)

Sumatra

Engano

Engano

Sumbawa

Lombok

Tanah Djampea

? Celebes

Luzon

Perak

Bazilan

India

Aru

Aru

Key

Dutch New Guinea

Waigeu

Germ. "New Guinea

Dammer (male)

Babber (female)

Nias

N. Borneo

Hainan

Palawan

Palawan

Bazilam

W. Sumatra

Bawean

Batu

Kangeama

Sula

Celebes

Nias

Sandakan

Sumatra

IV. Sumatra

Palawan

Balu

Lombok

Assam

Assam

Tonkin

Upper Burma

Sikkim

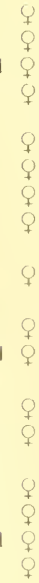

ษ

t

†

$1 \mathrm{C}$

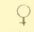

14

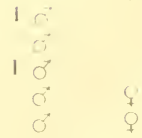

రా

के

18

18 


\section{Pieridae (Conlinued)}

Appias lalage aormis indra thrasea aristoxemus menandrus thoromion statilia

nupta pandione moira

$$
\text { ozolia }
$$

ada f. cana

pandana

nerva

$$
\text { ") f. tristitia }
$$

dunaetha

thasia

chorasina

xenia

heremia

ribbei

monna

protina

arbina $f$. semifrava

$$
\begin{aligned}
& \text { pancheia } \\
& \text { f. punctata } \\
& \text { f. virilis } \\
& \text { f. subochracea } \\
& \text { f. umbratilis } \\
& \text { f. citronella } \\
& \text { f. citrina } \\
& \text { infuscata }
\end{aligned}
$$

ab. saweloides

sosuas

ega sawela

tambora

f. melanides

f. limbata

antoniae

pseudoleis

pione

minata

terentilia

$$
\begin{aligned}
& \text { f. marginata } \\
& \text { f. aegina } \\
& \text { f. iria } \\
& \text { f. flaminia } \\
& \text { f. horatia }
\end{aligned}
$$

athena

plaeteria
Malacca

Formosa

Formosa

Hainan

Siam

Trebandrum

Nias

Sumatra

W. Sumatra

Ceram

Panda

Waigeu

Waigeu

Rendova

Dorey (male)

Roon (female)

"Germ. "New Guinea

Halmaheira

Milne Bay

Rubiana

Solomons

Fergusson

India

Palawan

Bawe an

Bawe an

Lombok

Lombok

Lombok

Lombok

Sumbawa

Sumbawa

Yule 1.

Lombok

Sumbawa

Batjan

Batjan

Ceram

Siam

Sumatra

Ishigaki

Palawan

Palawan

Palawan

Palawan

Palawan

Palawan

N. Borneo

Balabac

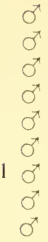

0

0

10

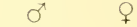

10

10

or

б

10

$\sigma^{x}$ 


\section{Pieridae (Continued)}

Appias ega mikomedera paulina yaksha

Saletara panda enganica

$$
\begin{aligned}
& \text { erebina } \\
& \text { hostilia } \\
& \text { martia } \\
& \text { chrysea } \\
& \text { margosa }
\end{aligned}
$$

c) cinna $f$. hastia chryselectra

liberia chrysoberylla obina 1) f. pseudcorinna

Huphina nama hainanensis

$$
\begin{aligned}
& \text { pitys aeliana } \\
& \text { " aelia } \\
& \text { " lucia } \\
& \text { " pityna } \\
& \text { " bolana } \\
& \text { " mithra } \\
& \text { " chrysopis } \\
& \text { mentes hierocles } \\
& \text { kühni Rober } \\
& \text { wetterensis f. allina } \\
& \text { perimale yuleana } \\
& \text {, hiscia } \\
& \text { nerissa corva } \mathrm{f} \text {. corvina } \\
& \text { nerissa physkon } \\
& \text { pimnata aurulenta } \\
& \text { " sorol } \\
& \text { " filiola } \\
& \text {, filia } \\
& \text { aspasia orantia } \\
& \text { " irina } \\
& \text { " zisca } \\
& \text { " pliokaia } \\
& \text { aspasia aspasina } \\
& \text { " remnia }
\end{aligned}
$$

lea malyra

hespera $f$. montana

meridionalis

natuna

naomi f. pseudamba

aga

oberthuri Rôber

Metaporia soracta denigrata

Pieris melete juba
Bazilan

India

Engano

Palawan

Balabac

Bazilam

Great Nicobar

Mindanae

Dutch New Guinea

Aru

Buru

Obi

Obi

Hainan

Formosa

Bawean

Kangean

Sumbawa

Dammer

Key

Fergusson

Mafor

Yule 1

Kabia 1.

Wetter

Yule 1.

Woodlark

Java

Lombok

Bangkei

Sula

Sula Besi

S. Celebes

Mindanao

Jolo

Bazilam

Balabac

Obi

Negros (male)

Mindoro (female)

? Singapore

1. Borneo

S. Borneo

Natuna

Lombok

Sumbawa

Mauncia (? Flores)

India

Japan 


\section{Pieridae (Continued)}

Pieris megamera

rapae lysicles

" yokohamae

nicipsa

f. atomaria

leucosima f. vaga

eumorpha

napi f. gratia

" leovigilda

n meridionalis

athenaia

mafini rossi $f$. farpa

$$
\text { 1) f. asta }
$$

Anapheis java $f$. magniplaga

teutonia savuana

$$
\begin{aligned}
& \text { ina } \\
& \text { anita } \\
& \text { micronesia }
\end{aligned}
$$

Aporia erataegi aderpal

$$
\text { " basanis }
$$

Euchloë daplidice ambicola

$$
\begin{aligned}
& \text { avidia } \\
& \text { plaeclara } \\
& \text { laenas }
\end{aligned}
$$

Elobina pasarga

mutiurcula

hypatia cada

lutana

andropia hydatis

namatia

Parelodina effeminata

$$
\begin{aligned}
& \text { anticyra } \\
& \text { invisibilis }
\end{aligned}
$$

Phrissura aegis gerasa

$$
\begin{aligned}
& \text { " nivata } \\
& \text { xiphia aebutia } \\
& \text { " malayana } \\
& \text { " fumigata } \\
& \text { " comma } \\
& \text { " georgis } \\
& \text { " terentia }
\end{aligned}
$$

Leptosia xiphia aebutia

Prioneris clemanthe euclemanthe

$$
\begin{aligned}
& \text { themana } \\
& \text { sacnia } \\
& \text { helferi }
\end{aligned}
$$

thestylis hainanensis

\section{Japan \\ Oshima \\ Japan \\ Japan \\ Dalmatia \\ Smyrne \\ Tian-Shan \\ Salêve \\ Savoy \\ Rome \\ Generoso \\ Rome \\ Istria \\ Sumba \\ Savu \\ Kisser \\ Wetter \\ Fiji \\ Sapporo}

Alpes-Maritimes

Kashgar

Tsintau

Vallée du Tong-ho

Palestine

"Germ. "New Guinea

Milne Bay

Waigeu

Key

New Guinea

Waigeu

Germ. "New Guinea (male)

Dutch New Guinea (female)

Roon

Wetter

Sula

Palawan

S. Celebes

Tanah Djampea

Padang

Lombok

Babber

Luzon

Bazilam

Hainan

Sumatra

Annam

Tonkin

Hainan
19

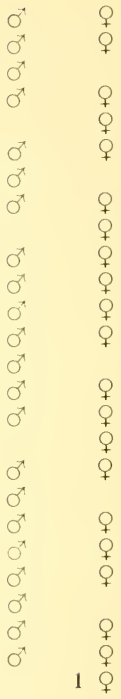

ర

o

$\sigma$

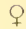

q

10

0

o

10

0

0

0

$0^{x}$

o

o

10

$\sigma^{x}$

o

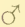

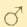




\section{Pieridae (Continued)}

Prioneris thestylis $f$. namilia

$$
\begin{array}{lll}
\text { " } & \text { n } & \text { formosana } \\
\text { " } & \text { " } & \text { malaccana } \\
\text { " } & \text { jugurtha }
\end{array}
$$

Synchloë belia melisande

$$
\text { n cardamines sajoura }
$$

Ixias salangana

" verna $f$. annamitica

- tonkiniana f. denigrata

singalensis $f$. connectens

$$
\text { ") f. nivescens }
$$

hainana

Leucophasia sinapis spirabilis

$$
\text { " " elvina }
$$

Dercas verhuelli menandrus

$$
\begin{aligned}
& \text { " f. lineata } \\
& \text { verhuelli pallidus } \\
& \text { lycorias } \mathrm{f} \text {. difformis } \\
& \text { amintha formosana }
\end{aligned}
$$

Hebomoia glaucippe anamandra

$$
\text { formosana }
$$$$
\text { liukiuensis }
$$

liukuensis f. conspergata vincia

ceylonica

erinna

aturia

leucippe daemonis " leucognia

celebensis f. sublustais

f. icterucus

Catopsilia flava $f$ crocalina

" $f$. jugurthina

n f. rivalis

celebica $f$. ostentata

scylla $f$. ascylla

$$
\text { cidra }
$$

saylloides

praerubiba

f. minavia

f. bangkejana

moluccarum

crocale micromalayana
1) $f$. virosa
f. citronella

Hainan

Formosa

Malacca

Siam

Palestine

Siberia

Salanga

Annam

Tonkin

Ceylan

Ceylan

Hainan

Saratow

Griechenland

Bhutam (male)

Sikkim (female)

Tonkin

Padang

Assam

Tonkin

Formosa

Kalao

Formosa

Oshima (female)

Okinawa (male)

Okinawa

Ishigaki

Ceylan

Luzon

Malacca

? Singapore (female)

Ceram

Buru

N. Celebes

S. Celebes

Saparua

Obi

Halmaheira (female)

N. Celebes

Lombok

Sumbawa

Babber

Flores

Natuna

Bangkei

Saparua

Sumba

Sumba

Babber
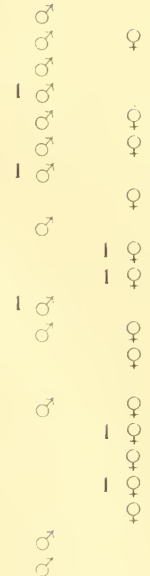

q

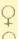

$0^{x}$

\%

or
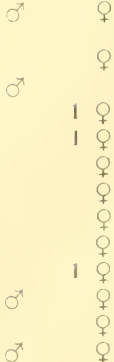


\section{Pieridae (Continued)}

Catopsilia pomona perspicia gorgophone f. siccias

f. nivescens

catilla $f$. bidotata

Colias phicomone phila

poliographis $f$. napata

f. murina

hyale palilis

" nankrakis

fieldi calidia

Gandaca harina hainana

$$
\begin{aligned}
& \text { gardineri } \\
& \text { dohertyi } \\
& \text { austrosundana } \\
& \text { aiguina } \\
& \text { ocalea } \\
& \text { samanga } \\
& \text { palawanica } \\
& \text { olis } \\
& \text { aurifua }
\end{aligned}
$$

Terias libythea awidia

$$
\begin{aligned}
& \text { candida micromalayana } \\
& \text { libera } \\
& \text { dindymene } \\
& \text { octogesa } \\
& \text { natuna } \\
& \text { senapati } \\
& \text { cantideva } \\
& \text { acandra } \\
& \text { arsapia f. apharia } \\
& \text { arsakia } \\
& \text { saraka } \\
& \text { cungata } \\
& \text { ? sonombina }
\end{aligned}
$$

hecabe sankapura

$$
\begin{aligned}
& \text { ? chennus } \\
& \text { kalidupa } \\
& \text { tentyris } \\
& \text { borneensis } \\
& \text { enganica } \\
& \text { lorana } \\
& \text { tamiaphis } \\
& \text { satellitica } \\
& \text { jalendra } \\
& \text { sinda }
\end{aligned}
$$

N. Celebes

Sambawn

Wetter

Wetter

Caschmir

Japan

Japon

Siberia

Siberia

India

Hainan

Bazilan

Engano

Lombok

Waigeu (male)

Saparua (female)

Sula

S. Celebes

Palawan

N. Borneo

Sula

Collingwood Bay

Maumerie

Halmaheira

Obi

Waigeu

Ladang

Natuna

Lombok

Wetter

Hong-Kong

Formosa

Formosa

Dutch New Guinea

Amboina

Hatly

Bawean

Alor

Kalicupa

Tanah Djampea

N. Borneo (male)

S. Borneo (female)

Engano

Natuna

Bazilan

Nias

Palawan

S. Celebes 


\section{Pieridae (Continued)}

Terias hecabe bandana

$\begin{array}{lll}\text { n } & \text { " } & \text { asanga } \\ & & \text { nivaria } \\ \text { " } & \text { " } & \text { levuna } \\ \text { " } & \text { n } & \text { cota }\end{array}$

lacteola prabha

" yaksha

andersoni godana

lombokiana

sari obnobla

thyreus

latimargo sophronia

$$
\text { " } \quad \text { pylos }
$$

tilaha aebitia

$$
\text { " epicharma }
$$

tominia horatia

$$
\begin{aligned}
& \text { " porabja } \\
& \text { " fannia } \\
& \text { " haresa } \\
& \text { " battana } \\
& \text { " mangolina } \\
& \text { " besina }
\end{aligned}
$$

alitha sanama

$$
\text { 1) marosiana }
$$

alitha sangira

$$
\begin{aligned}
& \text { " bazelana } \\
& \text { " bozonia } \\
& \text { " } \quad \text { f. zamida }
\end{aligned}
$$

norbana ovinia

$$
\begin{aligned}
& \text { salegos } \\
& \text { anios } \\
& \text { depicta } \\
& \text { epilachontes }
\end{aligned}
$$

celebensis toala

$$
\begin{aligned}
& \text { enophtalma } \\
& \text { poetelia }
\end{aligned}
$$

Pareronia valeria palawana

$$
\text { persides }
$$

baweanica

tryptena

hainanensis

kangeana

niasica
Banda

Batjan (male)

Halmaheira (female)

Salomons

Fiji

Arfak (male)

Dorey (female)

Palawan

Natuna

Formosa

Lombok

Palawan (male)

S. Borneo (female)

Engano

5. Celebes

Sula

S. Celebes

Bazelam

?

?

S. Borneo

E. Celebes

Birnungka

Saleyer

S. Celebes

S. Mangola

S. Besi

Sula

S. Celebes

Sangir

Bazelam

Jolo

Lutungam I.

S. Celebes

N. Celebes

Halmaheira

Obi

Amboina

Sangir

S. Celebes

Sula Mangoli

Sula Besi

Palawan

Tonkin

Bawean

Sumba

Hainan

Kangean

Nias 


\section{Pieridae (Continued)}

\begin{tabular}{|c|c|c|}
\hline Pareronia & Valeria & sundana \\
\hline n & n & sumbawana \\
\hline$"$ & phecae & ea criomena \\
\hline$"$ & argolis & argolira \\
\hline$n$ & " & f. radenides \\
\hline$" 1$ & tritaea & barjylia \\
\hline u & $n$ & ? hernecinia \\
\hline .. & tritaea & f. caecinia \\
\hline 4 & avatar & tarina \\
\hline " & $n$ & paravatar \\
\hline ". & jobaea & obiana \\
\hline 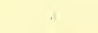 & $"$ & elsa \\
\hline it & $n$ & aebeja \\
\hline " & " & aviena \\
\hline "1 & $"$ & luceria \\
\hline$\pi$ & ” & luceria $f$. $g r$ \\
\hline
\end{tabular}

Lombok

Sumbawa

Bazelam

Buru

Batjan

S. Celebes

Bangkei (male)

? (female)

E. Celebes

Sikkim

Lower Burma

Obi

Ceram

Buru

?

Waigeu

Waigeu

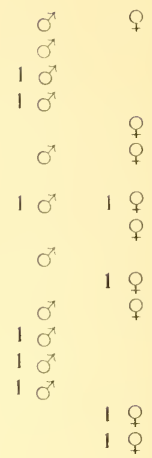




\section{PIERIDAE (Américan)}

Pereute charops columbina

$$
\begin{aligned}
& \text { meridana } \\
& \text { permagnus }
\end{aligned}
$$

leucodrosima beryllina bellatrix

$$
\text { autodyca bardela }
$$

Archonias bellona phaloreia

$$
\text { " sabrina }
$$

critias $f$. hades

"r aregillus

1. papilionides

" uniplaga

pharnakia

Charonias theano mychanus

$$
\text { 1) sorix }
$$

Catasticta bithys flisina

$$
\text { " " béthyna }
$$

Pieris buniae pharetia

$$
\text { sevata timotina buniae gargara }
$$

ausia $f$. lusella

amaryllis protazia

josepha $f$. gervasia

sevata tibirtia

locusta lubecula

richardi

1) molione

lorena perunota

$$
\text { " jumena }
$$

pyrrha ? riscorsa

$$
\text { stligitata }
$$

mantineia

austrania

calydonia f. magna

demophile niphates

charopis

miniotes

nissias

Leptophobia eleone comica

$$
\text { i) luca }
$$

tovaria marnga

$$
\text { gina }
$$

cinnia faledra

stannata messara

subarjentea lia

pinara oiantheia

agmara
Colombia

Venezula

W. Mexico

Ecuador

Peru

Rio Grande

Peru

Argentine

Colombia

Ecuador

Honduras

Bresil

Peru

S. Paulo

Bahia

Colombia

Blumenau

Peru

Matto Grosso

Bresil

Honduras

S. Mexico

Guatemala

Peru

Ecuador

Paraguay

Bolivia

Colombia

Peru

Bahia

Bresil

Honduras

Argentine (male)

Bolivia (male)

Honduras

Para

Rio Uaupes

Bahia

Paraguay

Colombia

Bolivia

Ecuador

Peru

Colombia

Peru

Bolivia

Peru

Bolivia

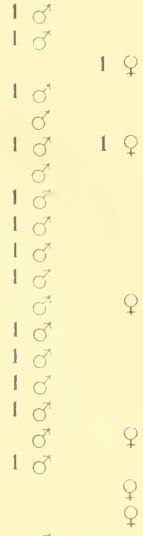

19

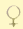

10

$$
1
$$




\section{Pleridae, American (Continued)}

1)aptoneura lycimnia phalzania

$$
\text { pantoporia }
$$

narmia

gargaphia

cubana

polyhymnia f. phaenna

petrenia

" f. danlia

donata

i) f. bianca

myrtis

maotes

iphigenia

eurymnia $t$. asta

aelia $f$. pistoria

$f$. theodora

laria louisella

peruviana regnidas

f. yolanda

isandra kleta

peruviana galatia

Hesperocharis nera nervana

\section{amazonica}

nilios

" f. aphaia

nereina elea

marchalli coloe

Cathaemia hirlanda phanasia

$$
\text { praeclara }
$$

Colias lesbia eurinice

dinora goryna

Dismorphia nomesis Obola

chara

cretacea vessela

$$
\text { rovya }
$$

thermesia margaritacea

lemnorina logoja

marionides

melia moena

astynome cellularis

amphione amphionides

melite defigurata

critomedia mudana

critomedia cereda
Bahia

Matto Grosso

Matto Grosso

Bresil

Cuba

Colombia

S. Bresil

S. Bresil

Bolivia

Bolivia

Peru

Peru

Tarapote

Colombia

Ecuador

Rio Uaupes

Peru

Ecuador

Peru

Honduras

Bolivia

Bolivia

lquites

Rio Uaupes

Rio Laupes

Bolivia

Peru

Matto Grosso

Bresil

Rio Grande

Peru

Ecuador

Mexico

Bresil

Minas Ceraes

S. Catharina

Bresil

Blumenau (male)

Rio Grande (female)

Bresil

Bahia

Ecuador

Peru

Colombea

Rio Grande

Peru 


\section{ACRAEIDAE}

Actinote pellenea lavonica guatemalena leitus

Parebra vesta sordice

$$
\begin{aligned}
& \text { " vestalina } \\
& \text { " formosana } \\
& \text { 1. alticola }
\end{aligned}
$$

$$
\text { n) }
$$

Miyana andromache agena moluccara pella

Limnas chrysippus canarensis

Danaida philene $f$. luxurians

$$
\begin{aligned}
& \text { transfuga } \\
& \text { subnubila } \\
& \text { uzos } \\
& \text { bonguensis } \\
& \text { molyssa } \\
& \text { woodlarkiana }
\end{aligned}
$$

plexippus niasicus

$$
\text { partita }
$$$$
\text { wetterensis }
$$

tychius

telmissus

$$
\text { f. gighion }
$$

melanippus umbrosus indicus ab. lotina

affinis malayana

$$
\begin{aligned}
& \text { n kaweiensis } \\
& \text { tambora } \\
& \text { coriacea }
\end{aligned}
$$$$
\text { fuliginosa } \mathrm{f} \text {. astakos }
$$$$
\text { strephon }
$$$$
\text { galacterion }
$$$$
\text { attruoides }
$$$$
\text { decentralis }
$$$$
\text { sangira }
$$$$
\text { taruna }
$$

mytilene kiriwina

$$
\begin{aligned}
& \text { fergussonia } \\
& \text { pittakus } \\
& \text { pleistarchus } \\
& \text { rubrica }
\end{aligned}
$$

lotis mezentius

- ismare felicia

$$
\text { goramica }
$$

Honduras

Bresil

Tenasserim

Annam

Formosa

Padang (male)

Seloc (female)

?

Woodlark

Teneriffe

Saparua

Batjan

Ternate

Obi

"Germ. "New Guinea

Collingwood Bay

Woodlark

Nias

Lombok

Wetter

Saleyer

Buton

Annam

Batu

Lower Burma

Natuna

Siam

Java

Sumbawa

Alor

Bawean

Etna Bay

Fergusson

Talaut

Bangkai

Sangir

Teruna

Kiriwna

Fergusson

New Guinea

Hatley

Palau

Balabac (male)

Sandakan (female)

Buru

Cioram 


\section{Acreaidae (Continued)}

Tirumala limniace cartissa

mutina

marsonia

mylasa

donia

melissa dravidarum

rufiventris

palawana

gariata

singaria

paryadres

phrynichus

pasatia

arikata

Parantica agla phormis

n maghaba

eryx meanius

serilus

" n erycina

Asthipa aspasia caulonia phargalia

rita

sherfordi

cervilla

cleona tigrana

luciplena

lurida

kirbyi decolorata

Asthipa melusine cythion

$$
\text { siris }
$$

schenki distrata

1) toxens

" periphas

vitrina odyssae

Caduga tytia tira

Radena juventa taiwaya

$$
\begin{aligned}
& \text { lipussa } \\
& \text { tontoliensis } \\
& \text { sophelisen } \\
& \text { sequana } \\
& \text { satellitica } \\
& \text { mincia } \\
& \text { phana } \\
& \text { stictica } \\
& \text { callatia } \\
& \text { lycosura } \\
& \text { 7itah }
\end{aligned}
$$

Formosa

Ceylan

Hainan

Kalao

Lombok

Malabar

Nias

Palawan

N. Irelande

Dammer

Key

New Guinea

Siam

Sula Besi

Tonkin (male)

Annam (male)

Formosa

?

N. Borneo

Nias

Bali

W. Sumatra

Bawean

N. Borneo

Palawan

Halmaheira

N. Celebes

Sula

"Germ. "New Guinea

Yule 1.

"Germ. "New Guinea

Wetter

Dutch New Guinea

Milne Bay

Samar

Agra

Gent. Celebes

Bangkei

N. Celebes

Sula

Birnungka

Saleyer

Bawean

Lombok

Sumbawa

Kalao

Ronerate

Natuna 


\section{Acreaidae (Continued)}

$$
\begin{array}{ccl}
\text { Radena } & \text { juventa } & \text { kinitis } \\
\text { " } & \text { " } & \text { georgina } \\
\text { " } & \text { " } & \text { tanais } \\
\text { " } & \text { " } & \text { catella } \\
\text { " } & \text { " } & \text { piadina } \\
\text { " } & \text { piada } \\
\text { " } & \text { eugeniz } \\
\text { " } & \text { " } & \text { metaxa } \\
& \text { " } & \text { zanura }
\end{array}
$$

Radena juventa ribbeı

$$
\begin{aligned}
& \text { galaecia } \\
& \text { goria } \\
& \text { lirungensis } \\
& \text { messana } \\
& \text { lesora } \\
& \text { sumbawana } \\
& \text { ellida } \\
& \text { ogylla }
\end{aligned}
$$

Similis juventa tragaca

$$
\begin{aligned}
& \text { f. hyria } \\
& \text { macrina } \\
& \text { interposita } \\
& \text { vulgaroides } \\
& \text { megaloides } \\
& \text { ditiones }
\end{aligned}
$$

Caduga melaneis plataniston

$$
\text { szechuanus }
$$$$
\text { sinopion }
$$

banksi mnasippis

Nectaria durvillei metris

? munaensis

blanchardi phlegeton

$$
\begin{aligned}
& \text { kuihni Rôber, } \\
& \text { garunda } \\
& \text { djampeana }
\end{aligned}
$$

durvillei keyensis

$$
\text { aruna }
$$$$
\text { nika }
$$

leuconoe missilia

$$
\text { moira }
$$$$
\text { vedana }
$$

hypermnestra hera

arbela

belina
N. Borneo

Colling Wood Bav

Mafor

Hatley

Dutch New Guinea

Dutch New Guinea

New Guinea

Germ. "New Guinea

Bougain Ville (female)

?

N. Ireland

(male)

Philippines

Bazilan

Talaut

Mindanao

Flores

Sumbawa

Obi

$\mathrm{x} \times \mathrm{x}$

Okinawa (male)

Ishigaki (femalc)

Annam

Sumatra

Natuna

Java

Nias

Batu

Sikkim

Sze-Chuan

Malacca

Batu

Salawatti

Buton

Kalidupa (male)

E. Celebes

Bonthain

Tanah Djampea

Key

Aru

Dorey

Okinawa

Bazilan

Talaut

Batu

Sumatra

N. Borneo

S. Borneo

Obi

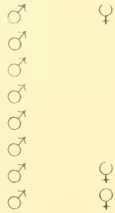

$\begin{array}{ll}\pi & f \\ 5 & q \\ 0 & f \\ 0 & q\end{array}$

$q$
9
$q$

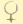

T 


\section{Acraeidae (Continued)}

Nectaria aza hertha

$$
\text { " } \text { " vosseleri }
$$

Hestia lynceus thalassia

$$
\text { favorinos }
$$

fumalata niasica

logani arcine

$$
\begin{gathered}
\text { " arceste } \\
\text { vingo } \\
\text { " diana } \\
\text { jasonia } f \text {. diabolica }
\end{gathered}
$$

Ideopsis laos somia

$$
\begin{aligned}
& \text { " natunensis } \\
& \text { " peracana } \\
& \text { " lingana } \\
& \text { " ardana } \\
& \text { cestalis batuna }
\end{aligned}
$$

Ideopsis vitrea arachosia

$$
\begin{aligned}
& \text { mortaica } \\
& \text { arfakensis } \\
& \text { nebis }
\end{aligned}
$$

inuncta iza
Sula

Auboina

Naluna

N. Borneo

S. Borneo

Nias

S. Borneo

Natuna

N. Borneo

Batu

Ceylan

Sumatra

Natuna

Malacca

Linga

N. Borneo

Batu

S. Celebes

Morotai

Arfak

Buru

Sula

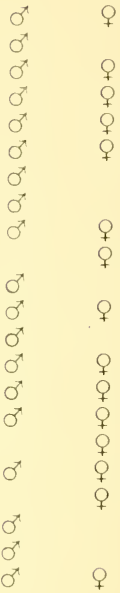

ITHOMINAE

Minas Geraes 


\section{EUPLOEINAE}

Euploea climena bandana

$$
\begin{aligned}
& \text { " valeriana } \\
& \text { compta virudha } \\
& \text { " eucompta } \\
& \text { palmedo adorabilis } \\
& \text { deheeri suavissima } \\
& \text { " salinator } \\
& \text { werneri } \\
& \text { aethiops lygdania } \\
& \text { " pheres } \\
& \text { " coffea } \\
& \text { menaeses } \\
& \text { tarnis }
\end{aligned}
$$

Euploea batesi publilia

$$
\begin{aligned}
& \text { minucia } \\
& \text { pinaria } \\
& \text { leonata } \\
& \text { cerberus subpunctata } \\
& \text { guerini tenebrosa } f \text {. amethysta } \\
& \text { melanopa albifions } \\
& \text { delicia } \\
& \text { parca } \\
& \text { potaissa } \\
& \text { cissia } \\
& \text { julica } \\
& \text { zana } \\
& \text { nesis } \\
& \text { t. sardes }
\end{aligned}
$$

Euploea eleutho eleutheria
Banda

Roma

Damme:

Babber

Wetter

Lombok

Alor

Vulcan is

Nafor

Jobi

Germ. "New Guinea

Dutch New Guinea

Dutch New Guinea

Germ. "New Guinea

Dutch New Guinea

Waigeu

New Guinea

N. Ireland

Germ. "New Guinea

Dutch New Guinea

Dutch New Guinea

Germ. "New Guinea

Salawatti

Mlisol

Waigeu

Mafor

Roon

Dammer

N. lreland

hirwina

Ceram

Batjan

Treasury I

Rendova

Dutch New Guinea

Dutch New Guinea

Dutch New Guinea

Obi

Babber

Teon

Palawan

Salawatti

Letti

Letti

Roma

Wetter

Sula

Bazilan 
Euploeinae (Continued)

Euploea alcathe easatia

martinis

vorsara

salistra

gardineri

simplex

lucania

dione epiphanea

$$
\begin{array}{ll}
\text { " massina } \\
\text { " sapitana } \\
\text { " pasina } \\
\text { " f. avida } \\
\text { " menodice }
\end{array}
$$

modesta deriopes

confusa faunia

catana

melia

confusa japudia

wallacei gilda

$$
\text { ares }
$$

helcita matilica

lilybaea

aglaina

spiculifera pydna

radica

resarta turbeina

curianas за cumaxa terentilia

anmymone hadrumetus

prinosa tersaica

bauermanni kalaona

orantobates

godarti $f$. defigurata

f. cernosa

f. dominans

diocletianus $f$. despoliata areithous

nemertes lycolon

pseudohisme f. trysa

quintia

nessia

oppia

nemertes christina

misolensis

oeneon

sexguttata

offaka
Siam

Sumatra

Sumatra

Natuna

Pera

Nias

Batu

Sumatra

S. Borneo

Lombok

Batu

Burma

Sambawan

Hainan

Dutch New Guinea

Germ n New Guinea

Fergusson

Dutch New Guinea

Obi

Buru

Thaiti

N. Hebrides

Nahé

Obi

Obi

New Guinea

- Germ. "New Guinea

Fergusson

Hainan

Hainan

Kalao

Chantabun

Upper Burma

Saigon

Siam

Annam

Natuna

Ceram

Obi

Fergusson (male)

Milne Bay (male).

Woodlark

Mafor

Salawatti

Misol

Hatley

"Germ. "n New Guinea

W aigeu 


\section{Euploeinae (Continued)}

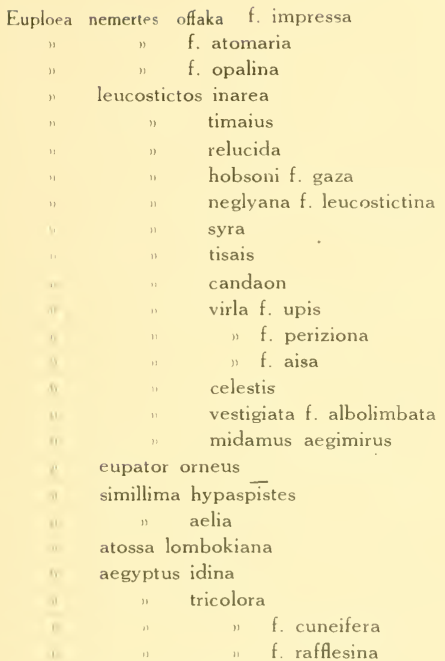

diana tombigensis laodikeia

asyllus laurentia

aegyptus limyrus

assimilata bandanensis

f. nepotina

usipetes astrifera

" $f$. albodiscalis

rezia $f$. padilla

usipetes roeberi

vollenhovi aganor

anitra

eleusina palata

phenarete vitrina volanda

corus defiguratus

" statius

celebica

nikrilon

f. pauperata

f. luxurianta

althaea juvia

treitschkei decia
Waigeu

- Germ. - New Giunca

New Guinea

Batu

Bawean

Bali

Formosa

Hainan

N. Borneo

Lombok

Sumbawa

N. Celebes

S. Celebes

S. Celebes

Tonkin

Sumatra

Hainan

S. Celebes

Bazilan

Sandakan

Lombok

Kanjan

Bawean

Bawean

Bawean

E. Celebes

Saleyer

Shortland 1

Batu

Banda

Banda

Germ. "New Guinea

Fergusson

Milne Bay

Dutch New Guinea

Bangkei

C. Celebes

E. Celebes

Lower Burma

Sula

Bali

Sumatra

N. Celebes

Bawean

Amboina

Amboina

Formosa

Milne Bay 


\section{Euploeinae (Continued)}

Euploea treitschkes f. pulverulenta

eugenia

gamelia astiana

treitschkei aebutia

$$
\text { caelia }
$$

clausina hygina

offaka

tulliolus nocturna

mardonia

ecbatana

meda

koxinga

hyacinthus mangolina

$$
\begin{aligned}
& \text { subcongeia Rôber. } \\
& \text { besinensis }
\end{aligned}
$$

pumila salpingoides
f. amida
f. anaitis
f. melitta
f. sublucinda
kirschi $f$. amantia
f. parvipunctata
bismarckiana
garcila

jamesi flaminia

hymens catolina

kühniana

liza

aga

ornata

menamoides

visenda lonia

wetterensis

sada

mazares mazarina

palawana

monilina

natunensis

lombokiana

philina

angiana

hopfferi helia

pyres flaninifela

cledonia

obiana

paratellis
Germ "New Guinea

Vulcan I.

W. Sumatra

Jobi

Mafor

Sumbawa

Waigeu

Salawatti

Milne Bay

Philippines (male)

Bazelan (female)

Pura

Formosa

Sula

N. Celebes

Sula Besi

Germ. "New Guinea

Germ. "New Guinea

Germ. "New Guinea

Germ. n New Guinea

Germ. "New Guinea

Waigeu

Waigeu

Mioki

Dutch New Guinea

Salawatti

Dammer

Roma

Babber

Letti

Key

Babber

Babber

Wetter

Wetter

W. Sumatra

Palawan

Bazelan

Natuna

Lombok

Adonara (male)

Pura (female)

Flores

Banda

Solomons

Ceram

Obi

Batjan 


\section{Euploeinae (Continued)}

Euploea trimeni duilia gerderi phoebaris " seraphita

mulciber barsine

timora

wetterensis

doleschalli amarynceus

ternis

limbata

lacordairei mica

dotata

harrisi $f$. depunctata

f. discalis

cassia

invitabilis

baweanica

doleschalli agema

gloriosa pompilia

dufresne azaga

crameri nagasena

tenggerensis

singarhada

lanista
Obi

Lombok

Bazelan

Formosa

Sumatra

Wetter

Salawatti

Jobi

Waigeu

Engano

Palawan

Siam

Siam

Lombok

Alor

Bawean

Germ. New Guinea

S. Celebes

Philippines

Batu

E. Java

Bali

Natuna

PALAEOTROPINAE

Tellervo zoilus antipatrus

$$
\begin{aligned}
& \text { hiempsal } \\
& \text { vereja } \\
& \text { limetanus } \\
& \text { meforica } \\
& \text { niveipicta } \\
& \text { roonensis } \\
& \text { jobinus } \\
& \text { sariapus } \\
& \text { pantanus } \\
& \text { zephoris }
\end{aligned}
$$

Milne Bay

Germ. "New Guinea

Germ. "New Guinea

Dutch New Guinea

Mafor

Key

Roon

Jobi

Fergusson (male)

Kirwina (female)

Yule I.

Germ. New Guinea 


\section{NYMPHALIDAE}

Symbrenthia intricata

$$
\begin{array}{cl}
\text { brabira scatinia } \\
\text { hippoclus clausus } \\
\text { " } & \text { confluens } \\
\text { " } & \text { obianus } \\
\text { " } & \text { batjiana } \\
\text { " } & \text { anscho } \\
\text { " } & \text { buruanus } \\
\text { " } & \text { atta }
\end{array}
$$

Parthenos sylvia etega

$$
\begin{aligned}
& \text { fusciplena } \\
& \text { neaohannoverana } \\
& \text { pherekrates } \\
& \text { obiana } \\
& \text { tualensis } \\
& \text { numita } \\
& \text { pherekides } \\
& \text { theriotes } \\
& \text { guinensis } \\
& \text { sylvicola } \\
& \text { philippensis } \\
& \text { guttata } \\
& \text { bangkeiensis } \\
& \text { joloensis } \\
& \text { sulana } \\
& \text { ca terentina } \\
& \text { ? cyniluous } \\
& \text { risius sumatrensis } \\
& \text { 1) bellimontis }
\end{aligned}
$$$$
\text { nodrica terentina }
$$$$
\text { tigrina ? cyniluous }
$$$$
\text { gambrisius sumatrensis }
$$

Hestina nama subdecorata nama mamida

Euripus halitherses ff hadrua

$$
\begin{aligned}
& \text { f. gulussa } \\
& \text { f. neda } \\
& \text { f. gyrtone } \\
& \text { f. phygalia } \\
& \text { sumatrensis }
\end{aligned}
$$$$
\text { f. haterius }
$$

mastor

borneensis $f$. crastiana

f. uniformis

niasicus

palawanicus

f. acara
N. Celebes

Formosa

N. Celebes

S. Celebes

Obi

Batjan

Sula

Buru

Fergusson

Guadalcanar

?

N. Hannover .

Fergusson

Obi

Key

Goram

Milne Bay

Kollingwood Bay

New Guinea

Nias

Bazilan

Dutch New Guinea

Bangke :

Jolo

Sula

Dutch New Gumea

Dulch New Ciuinea

Sumatra

?

)

Sumatra

Tonkin

Sikkim

Tonkin

India

Tonkin

Sumatra

W. Sumatra

Tonkin

N. Borneo

N. Borneo

Nias

Palawan

Palawan

Siam 


\section{Nymphalidae (Conlinued)}

Euripus consimillis curimus $f$. amala

$$
\begin{aligned}
& \text { f. sunta } \\
& \text { f. diocletiana } \\
& \text { f. gudila } \\
& \text { f. torquella }
\end{aligned}
$$

Herona marathus marathon

$$
\text { djarana }
$$

Eulaceura osteria solentia

$$
\text { " nicomedia }
$$

Dichorragia nesimachis formosanus

$$
\begin{aligned}
& \text { neriotes } \\
& \text { pelurius } \\
& \text { derdas } \\
& \text { niasicus } \\
& \text { machates }
\end{aligned}
$$

Kuthalia vasanta $f$. fulica

garuta anagama

$$
\begin{aligned}
& \text { " meridionalis } \\
& \text { " suddhodana } \\
& \text { " appama } \\
& \text { " gurda }
\end{aligned}
$$$$
\text { purana }
$$

jahnu jahnides

$$
\text { jama jamida }
$$$$
\text { ") ocrena }
$$$$
\text { 1) cusama }
$$

alpheda yamuna

$$
\begin{array}{ll}
\text { " kenodatus } \\
\text { " krannon }
\end{array}
$$

aconthea nivepicta

eriphyle chula

$$
\text { " lioneli }
$$

lusiada mindorana

$$
\text { merta phatasma }
$$

merta f. pseud eriphyle

kanda atys

agnis madosta

$$
\text { " tinna }
$$

monima pseudosalia

$$
\begin{aligned}
& \text { f. viridibasis } \\
& \text { natuna } \\
& \text { ilka } \\
& \text { limbata }
\end{aligned}
$$

Siam

Siam

Siam

Siam

Siam

Tonkin

Nias

S. Borneo

W. Sumatra

Formosa

Japan

N. Celebes

S. Bornero

Nias

Sumatra

N. Ceylan

India

Malabar (male)

Coromandel (female)

Sikkim

Saigon (male)

Siam (female)

? (malè)

Perak (female)

Sumatra

Tonkin

Sikkim

Upper Burma

Mindanao

Malacca

Sumatra

S. Borneo

Lombok

Siam

Tonkin

Bazelan

Mindoro

?

Tenasserim

Sumatra

Sumatra

N. Borneo

Sumatra

W. Sumatra

IX'. Sumatra

?

N. Borneo

S. Bornen 


\section{Nymphalidae (Continued)}

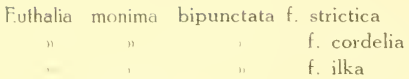

otthonis

kesava arhat sastra tidela

aphne ? pseudovalnikis

clathrata f. purpurea

cibaritis $f$. vinaya

aquamarina

leucotaenia dinorah

calliphorus smaragdifera

puseda $f$. floralis

diardi f. martini

ambalika f. paramitra

cocytina f. margiola

f. violetta

f. montigaga

ambalika f. arya

f. decolorata

f. trilobita

cocytus samsara

godarti nirodha

vacillaria $f$. arama

mara

lepidea miyana

stharvara

tayana

lepidea flaminio f. mirditta

julii indochinensis

" odilinia

teita sarmana

medaga

lubentina indica

chersonesia

mariae

philippensis

arasada

psitheucu.

aedonides

adenia sumatrana

sapitana

beata

princesa

pira
N. Borneo

N. Borneo

N. Borneo

Nias

Sikkim

Annam

Tonkin

N. Borneo

N. Borneo

Andamans

Philippines

Bazelan

Philippines

Malacca

Borneo

N. Borneo

Sumatra

Sumatra

Sumatra

N. Borneo

N. Borneo

N. Borneo

Nias

Bazelan

S. E. Borneo

Sumatra

India

Upper Burma (male)

Assam (female)

Tonkin (male)

Cochinchina (female)

Tonkin

Tonkin

Siam

Bazelan

Mindanao

Sikkim

Malacca

N. E. Sumatra

Bazelan

!ndia

Ceylan

S. Borneo

Sumatra

Lombok

Siam

Palawan

Bawcan

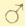

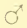

$0^{\pi}$

$\pi$

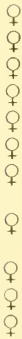

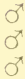

q

q

? 


\section{Nymphalıdae (Continued}

Euthalia evelina bolitissa

$$
\text { vallona }
$$

compta

mahonica

magama

formosana

armanda cutaenia

anasia yenadora

dunya manaya

sahadeva nadaka

kosempois.

laja f. galana

phemius selitzi

mahadeva zyk.i...

sakii

sericea

aetion danata

" sesitheus

nara alutoya

undosa

shinnin

Tanaecia aruna f. satapana pratyecha munda f. calina

sumatrana

nyagredha

f. Carma

paryanya $f$. irkranida

$$
\text { n }
$$

vikrama f. norina

pargluna balina

stryjiana

pela crowleyi

djataka

heliophila

Euthalia aeropus helvidius

paisandrus

choridorae

chorilus

eutychius

f. ergena

f. midia

f. albifera

hegias

Adolias sandakanus

dirtea $f$. esmalta jadeiteina
N. Celebes

Siam

Phang

W. Sumatra

N. Borneo

Formosa

N. Celebes

Java

W. Sumatra

Assam

Formosa

Hong-Kong

Malacca

Sumatra

Nias

Waigeu

Aroa (brit. New Guinea)

Sze-Chuan

Mou-Pin

Formosa

Malacca

W. Sumatra

S. E. Borneo

N. Borneo

Sumatra

Malacca

?

W. Sumatra

W. Sumatra

W. Sumatra

Bali

Lombok

N. Borneo

Natuna

Nias

Buru

Obi

Batjan

Waigeu

Dutch New Guineza

Germ. "New Guinea

Germ. New Guinea

New Guinea

N. Britain

Borneo

5. Borneo

lenasserin 


\section{Nymphalidae (Continued)}

Mlolias dirtca agosthena

elanor

javana

gigantea

elloca

Apaturina erminia ermina

mirona

octavia

antonia

Apatura ambica martini

chryselora

$$
\text { claribella }
$$

fulva setia

$$
\begin{aligned}
& \text { "f. pseudofasciela } \\
& \text { "f. pseudopallas }
\end{aligned}
$$

parisatis atacinus

$$
\begin{aligned}
& \text { javana } \\
& \text { borneana } \\
& \text { tonkiniana } \\
& \text { staurakius }
\end{aligned}
$$

nakula mindora

Mynes schônbergi Rôber.

$$
\begin{aligned}
& \text { geoffroi vanceckei } \\
& \text { " tulturulla } \\
& \text { " } \\
& \text { " ogulina } \\
& \text { " eugenius f. scatinia }
\end{aligned}
$$

Prothoe calydonia makasthana

$$
\begin{aligned}
\text { franki hanspices } \\
\text { " aphodite } \\
\text { " vilma } \\
\text { " nausikaa }
\end{aligned}
$$

australis $f$. guilelmi

$$
\begin{aligned}
& \text { decolorata } \\
& \text { mafalda }
\end{aligned}
$$

Limenitis procris patuna

agnata
sumbana
minoë
neutra
mentra
sumbawana
floresiana
arnoldi
indifragis

Siam

Tonkin

Java

Nias

Mindoro

Obi

Buru

Waigeu

Milne Bay

N. E. Sumatra (male)

Upper Burma (male)

Tonkin

Formosa

Tien-Tsuen

Formosa

Formosa

harwar

E. Java

N. Borneo

Tonkin

Hong-Kong

Mindoro

?

Germ. "New Guinea

Waigeu

Waigeu

Milne Bay

New Guinea

"Germ. "New Guinea

N. Borneo

Bazelan

Palawan

Siam

Tonkin

"Germ. "New Guinea

Misol

Milne-Bay

Batu •

S. Borneo

Sumba

Sumatra

W. Java

Lombok

Sumbawa

Flores

Bawean

Malabar (male)

Karwar (female) 


\section{Nymphalidae (Continued)}

Limenilis lyncida eutaenia lycone lyconides urdaneta mahastha marta avalskita populi golias " chinensis masiki eminens

daraxa viridicans 11 theoda hollandi lombokiana fylzia dudu ? ginamurtva camilla prodiga sibylla puellula glorifica

Lebadea martha malayana

$$
\text { pava }
$$$$
\text { natuna }
$$

Stibochiana nicaea subucula colesia rothschildi

Neptis pryeri jucundites

lucilla sinetasilis

$$
\begin{aligned}
& \text { fridolini } \\
& \text { insularum }
\end{aligned}
$$

hylas luculenta

$$
\begin{aligned}
& \text { cymada } \\
& \text { hatra } \\
& \text { satellitica } \\
& \text { sopatra } \\
& \text { terentia } \\
& \text { licinia } \\
& \text { flaminia } \\
& \text { sophaina } \\
& \text { vosama } \\
& \text { aloerica } \\
& \text { serapia } \\
& \text { jaculatrix } \\
& \text { sphaerica } \\
& \text { saleyera } \\
& \text { oda }
\end{aligned}
$$$$
\text { yessonensis }
$$

nandina formosana

" genatina $\mathrm{f}$. suslitina

" acalina

intermedia passerculus leucothoe averides illigera pia
S. Celebes

S. Celebes

Bazelan

Mindanao

Lama

Ta-Tsin-Lou

Wefter

Kanei

N. Borneo

Sumatra

Lombok

Flores

Formosa

Saleve

Saleve

Japan

Malacca

Batu

Natuna

Tenasserim

Nias

Formosa

Amur

Saratow

Japan

Formosa

Rioun

Batu

Bawean

N. Borneo

Jolo

Lombok

Sumbawa

Sumba

Flores.

Alor

Kalao

Dammer

S. Celebes

Saleyer

Japan

Sapawa

Formosa

Siam

Tonkin

Tsushima

Chang-Yang

Bazelan 


\section{Nymphalidae (Continued)}

Neptis fuluginosa serapica

$$
\begin{aligned}
& \text { ebusa laetitia } \\
& \text { " euphemia }
\end{aligned}
$$

ophiana martabana f. alicia

jumbah amorosa

$$
\begin{aligned}
& \text { tonkiniana } \\
& \text { nalanda }
\end{aligned}
$$

columella singa

$$
\begin{aligned}
& \text { lombokiana } \\
& \text { sumbana }
\end{aligned}
$$

magadja panuta

$$
\text { plantea }
$$

annamitica

phylansia

duryodana nesia

$$
\text { tullia }
$$$$
\text { enesia }
$$

nata eresina

" leucoporus

aletophone

egestas

natana

agathyllis

f. vasilis

sona tushita

" acala

acala f. pseudodipala

sona lutatia

" palibathra

heliopolis $\mathrm{f}$. anygra

shepherdi damia

$$
\begin{aligned}
& \text { donata } \\
& \text { graziella }
\end{aligned}
$$$$
\text { mastusia }
$$

satina $f$, damarete

nausicaa symbiosa

$$
\text { symosa }
$$

lydia

brebissoni metioche

prasini terentia maionia

mesogus

venilia glyceria

leucoion

obiana f. pseudovanescens

continia

tadena

glaucia
Sumatra

Bazelan

Jolo

Annam

Andamans

Tonkin

Ceylan

?

Lombok

Sumba

Nias

N. Borneo

Annam

Sumatra

W. Sumatra

Nias

Falawan

?

Tonkin

S. Java

S. Borneo

Nias

W. Sumatra

N. Borneo

Siam

Tonkin

Annam

Formosa

Bazelan

Batjan

New Guinea

Waigeu

Obi

Dutch Vew Guinea

New Guinea

Milne Bay

Dutch New Guinea

Waigeu

Waigeu

Wetter

New Guinea

Waigeu

Fergusson

Ternate

Obi

Halmaheira

$\mathrm{W}$ 'aigeu

N. lreland 
Nymphalidae (Continued)

Neptis venilia holargyrea

\begin{tabular}{|c|c|}
\hline & ") ? godelewa \\
\hline & grimberta \\
\hline & pseudovenilia \\
\hline & dikavikasi suavior \\
\hline 1 & vikasi tainiri \\
\hline " & " batuensis \\
\hline (2) & nitetis sannilia \\
\hline " & " predymus \\
\hline 4 & " gatanga \\
\hline i & kahoga \\
\hline is & lasala \\
\hline ter & arnandia mothlone \\
\hline s! & saphos \\
\hline iv & nycteus ilos \\
\hline 1 & sankara gesta \\
\hline re & anjana discerna \\
\hline 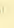 & " saskia \\
\hline 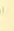 & miah digitia \\
\hline ii & "sarochoa \\
\hline 1 & helildora niasana \\
\hline$x$ & nirvana tawayana \\
\hline
\end{tabular}

Rahinda hordonia maligawa

$$
\begin{aligned}
& \text { senthes } \\
& \text { arcesta } \\
& \text { aigilipa }
\end{aligned}
$$

mysia sannians$$
\text { serpantina }
$$$$
\text { antara pytheas }
$$$$
\text { dindinga elea }
$$$$
\text { consimilis peria }
$$$$
\text { eurygrapha }
$$

consimilis stenopa

Eulepis athamas stratiocus lombokensis oitylus andamanensis

delphis delphinion dolon magniplagus schreiberi tisamonus hebe chersonesus

$$
\text { plautus }
$$$$
\text { " fallacides }
$$

Charaxes fabius cerynthus borneensis praestantius hierax $f$, rossa
Aru

Buru

Milne Bay

Dutch New Guinea

Cachgar (male)

Assam (female)

E. Java

Batu

Mindoro

Bazelan

Jolo

Nias

Nias

Tze-Chuan

Tze-Chuan

Amur

Tze-Chuan

Amboina

Nias

Amboina

Malacca

Nias

S. Celebes

Formosa

Sumatra

Lombok

Nias

Ternate

Halmaheira

S. Celebes

W. Sumatra

Australia

Waigeu

"Germ. "New Guinea (male

Dutch New Guinea (female)

Lombok

Lombok

Wetter

Andamans

S. Borneo

Assam

? (maie)

Malacca

Singapore

Nias

Ceylan

Malacca

Assain 
Nymphalidae (Continued)

Charaxes aristogiton peridoneus

$$
\begin{aligned}
& \text { polyxena } f \text {. pseudofervens } \\
& \text { " } \text { acolus } \\
& \text { " cybistia } \\
& \text { " crepax } \\
& \text { " f. parafervens }
\end{aligned}
$$

Amnosia decora eudamia f. flavilla

$$
\begin{aligned}
& \text { f. ochracea } \\
& \text { baluana } \\
& \text { petronia } \\
& \text { decorina }
\end{aligned}
$$

Pantoporia margaritha glorifica

$$
n
$$

$$
\begin{aligned}
& \text { nefte cordigera } \\
& \text { " } \text { astima } \\
& \text { " } \text { seitzi } \\
& \text { " } \text { subrata f. neftina } \\
& \text { " } \text { subratina } \\
& \text { " } \text { matthiola }
\end{aligned}
$$$$
\text { astura baelia }
$$$$
\text { " battakana }
$$$$
\text { , lactitincta }
$$$$
\text { " anaka }
$$

epalina parajina sulpitia tricula larymna agina

$$
\begin{aligned}
& \text { selessana } \\
& \text { subcurvata } \\
& \text { siamensis } \\
& \text { elisa }
\end{aligned}
$$

magnidana zilana

cama camasa

i) f. camida

zeroca galaesus meinippus

maena maenides

epimethis Bazelana

godmani reducta

selenophora ishiana

$$
\begin{aligned}
& \text { laela } \\
& \text { leucophryene } \\
& \text { batilda } \\
& \text { leucophryene } \\
& \text { caris } \\
& \text { f. epibaris } \\
& \text { rata moorei }
\end{aligned}
$$

rata cyma

kanwa xynara
Tonkin

N. Borneo

Sumatra

Natuna

?

?

W. Sumatra

W. Sumatra

N. Borneo

N. Borneo

Nias

Sumba

Lombok

Batu

Lower-Burma

Hong-Kong

Singapore

Sumatra

N. Borneo

Formosa

Sumatra

Java

N. Borneo

Lang-Bian

Formosa

Palawan

Badang

Nias

Siam

S. Borneo

Bazelan

Tonkin

Sikkim

Siam

Tonkin

Bazelan

Bazelan

Balabac

lshigaki

Formosa

Hainan

Tonkin

Hong-Kong

Sumatra

Sumatra

Singapore

Nias

Sumatra 


\section{Nymphalidae (Continued)}

Pantoporia kanwa napoleonus

n phorkys

ranga obsolescens

") karwara

abiasa matanga

parvara esra

i) helma

1) varina

" acutipennis

" indosinica

alcamone generosior

perus avitus

" hierasus

Argynnis sagana ilona

$$
\begin{aligned}
& \text { laodice ariana } \\
& \text { cydrana } \\
& \text { clara manais } \\
& \text { gemmata genia } \\
& \text { gong pereimnia } \\
& \text { trivia lathon } \\
& \text { protomedia argamia } \\
& \text { arcesia rucephala } \\
& \text { " karmana } \\
& \text { hyperbius sagada } \\
& \text { childreni caesarea } \\
& \text { paphia thalassata } \\
& \text { " neopaphia } \\
& \text { " megalegoria } \\
& \text { " f. valesenides } \\
& \text { " tsuchimana } \\
& \text { maga parsargades } \\
& \text { " chrysobelylla } \\
& \text { " seitzi }
\end{aligned}
$$

nerippe chlorotis

$$
\begin{aligned}
& \text { megalothymus } \\
& \text { nerippina }
\end{aligned}
$$

nerippe knanghsui anadiomena prasodies aglaia scarpheia

$$
\text { crassipunilates }
$$

madiana

f. myonia

bessa
Nias

Assam

Tonkin

Karwa

N. Borneo

W. Java

Padang

Nias

Assam

Tonkin

Mindoro

Lombok

Sumatra

Tsushima

Jns-Iki

tapan

Canton

Tien-Tsien

Sikkim

Ta-Tsien-Lou (male)

Tibet (female)

Langchowfu

Bosnia

(hang-Yang

Inn Shan (Chingan)

Munko Sardyx (Sajan)

Luzon

Ngeria

Saratow

Ampur

Sia-Lou

Tze-Chuan

Tsuchima

Alexanyeb (?) P'ersia

Teneriffa

Algeria

Nagasaki (male)

Japan (female)

Japan

Tibet (male)

Tze-Chuan (female)

Canton

Tshushima

China

Griechenland

Ussuri

Japan

Tse-Kou (male)

Sia-Lou (female) 


\section{Nymphalidae (Continued)}

Argynnis aslaia tardena

$$
\begin{array}{r}
\text { ". methana } \\
\text { niobe cisenna } \\
\text { " laranda } \\
\text { " f. mionia } \\
\text { adippe rielherte } \\
\text { " solitania } \\
\text { " garcila } \\
\text { " zaremna } \\
\text { " maimaria } \\
\text { " adelassia } \\
\text { lusiana fregsna } \\
\text { hecate teliburniana } \\
\text { " mantava } \\
\text { " centia }
\end{array}
$$

aphirape hermina

thera

pambetis

daphne epidaphne

inkator

ochrolonia

pales brogotarus

korla

axona

zetes

eupales

napalis

palina

amathusia bivina

halesa

bladireri

plalognana

serena

jugnetha

dinara

euphrosyne cynosama

calknota

nestor

peropara

theras

anka

densoi

ausonina

rusalka

orphamus
Ta-Tsuen-Lou

Castel

Klauson

Krain

Marseille

N. China (Tschifu)

Pekin

Saratow

Irkutsk

Klausen

Alpes-Maritimes

Tze-Chuan

Herzegovina

Krain

Saratow

Estland (male)

Dorpat (female)

Schawyv (Tannuoia)

Ussuri

Saratow (male)

? Klausen (female)

Martigny

Amur

Alpes-Maritimes

Korla

Nicolajewsk

Sajan

Tibet

Kuluk

Ta-Tsien-Lou

Saratow

Pyrénées

Fusie

Plalognan

Zermath

Tericlis (Karrasee)

Herzegovina

Salève

Fusio

Generoso

Ural

Scotland

Caukas

Dunes

Bessekeol

Saratow

Siberia (male)

Altai (female)

N. Engadine

N. Engadine




\section{Nymphalidae (Continued)}

\section{Argynnis ino eporeria}

$$
\begin{array}{ll}
\text { " } & \text { tergesta } \\
\text { " acrita } \\
\text { " tarnis } \\
\text { " pardicus } \\
\text { " siopelus } \\
\text { ". trachalus } \\
\text { " tigroides } \\
\text { " discolus }
\end{array}
$$

Melitaea cinxia arelatia

$$
\begin{aligned}
& \text { ocalia } \\
& \text { sacalia } \\
& \text { elis }
\end{aligned}
$$

asteria mevanda

aurinia volupis

$$
\begin{aligned}
& \text { " anglicana } \\
& \text { " bulgarica } \\
& \text { " lantosca }
\end{aligned}
$$

dia leonina

" hyda

thore hyperisia

hyperlampra

freija calis

phebe telona

$$
\text { zeluta }
$$$$
\text { ogygia }
$$

phoebe loklis

$$
\begin{aligned}
& \text { naranta } \\
& \text { koios } \\
& \text { sylleion } \\
& \text { minoa } \\
& \text { virginia } \\
& \text { gerenia } \\
& \text { rovia }
\end{aligned}
$$

degona phaisana

athalia nossis

$$
\begin{aligned}
& \text { noctula } \\
& \text { luriflia } \\
& \text { lachares } \\
& \text { scardona } \\
& \text { suessula } \\
& \text { limera } \\
& \text { bolis } \\
& \text { lucifuga } \\
& \text { tinica } \\
& \text { melida }
\end{aligned}
$$

Congo (male)

Courmajeur (female)

S. Esthland

Siberia

Ussuri

Altai

Kamschatka

Tian-Shan

Yokohama

Witien

Lagrave

Siberia

Saratow

Amasia

Glockner

Montelima

North Kent

Bulgaria

Laroche (male)

St. Martin (female)

Wallis

Juldus (huldsecha)

Amieux

Pyrénées

Schawar (Tannuola)

Palestina

Beyrouth

Griechenland

Saratow

Herzegovina

Klausen

Conne

Engadine

Alpes-Maritimes:

Lissabonne

Tessin

S. Tirol

Japan

Glarnisch

Salève

Sweden

Agram

Kochelsec.

Bosnia

Bulgaria

Saratow

Irkutsk

Generoso 


\section{Nymphalidae (Continued)}

Melitaea athalia longa

$$
\text { delminia }
$$

celadusa

parthenie ofella

melitona

gillon

sphines

didyma $f$. echrocea

oreithya

elawar

paphlagonia

onarea

naina

"f. andotropia

f. viridescens

tarlomia

scilennis

thyreatis

marsilia

dictynna alpestris

phyloa

salceda

praxilla

Ducapa fasciata ortopla

palloris

Ergolis ariatne pallidior

$$
\begin{aligned}
& \text { f. Jufotenila } \\
& \text { gedresia }
\end{aligned}
$$

Penthema michallati $f$. ponponia

$$
\begin{gathered}
\text { lisarda f. umbratila } \\
\text { "I mihintala }
\end{gathered}
$$

annamitica

$$
\text { f. telearchides }
$$

Calinaga buddha formosana

$$
\text { lactolis }
$$

Cethosia cydippe thymbrasa

aurigina

obiana

iphigenia

toxata

alkmene

cleanthis

woodlarkiana

cenchrites

herzeni

claudilla

praestabilis $f$. hermanni
Lôtschental

Klausen

Alpes-Maritimes

Castel

Alpes-Maritimes

Neuveville

Russia

Savoy

E. Kustenland

Dukdan (Sarafshan)

Transcaspia

Garm

Klausen

Lana

Klausen

Wallis

Alpes-Maritimes

Dunes

Marseille

Engadine

Klausen

Alpes-Maritimes

Krain

Bazelan

Palawan

Assam

Lombok

Alor

Tonkin

Formosa

Upper Burma

Annam

Annam

Formosa

Chang-lang (female)

?

(male)

Ceram

Batjan

Obi

Buru

Goodenough

Fergusson

Kirwina

Woodlark

?

Yule 1.

Dorey

? 


\section{Nymphalidae (Continued)}

Cethosia cydippe lucina

$$
\begin{gathered}
\text { " theona } \\
\text { penthesila pakcha } \\
\text { " filiola } \\
n \quad \text { exsanguis } \\
\text { "diffusa }
\end{gathered}
$$

obscura hormista

lamarckei f. jeusta

$$
\text { elateia }
$$

cyane euanthes

$$
\text { " cidaris }
$$

myrina melancholica

cydippe sarnada

biblis tisamena

tisamena f. mixta

$$
\text { f. viridiana }
$$

biblis hainana

$$
\begin{aligned}
& \text { phanaroia } \\
& \text { perakana } \\
& \text { narmada } \\
& \text { alcesta } \\
& \text { adantonia } \\
& \text { sandakana } \\
& \text { seramensis }
\end{aligned}
$$

hypsea triocala

$$
\text { " munjava }
$$

fruhstorferi Stich.

palawana

mindanaensis festiva

tambora floresana

$$
\text { 1) aptia }
$$

Cynthia arsinao f. ardea

$$
\text { buruana }
$$

figalia

polykaste

clorokusuna

pisidoke

adina

arsinoe rebeli

erota dajakorum

f. montana

erotala f. cantoré
Jobi (male)

Mafor (female)

Buru

Wetter

Sumba

Lombok

Sumbawa

N. Hannover

Babber

Koer

Tonkin

Siam (male)

Cochin-China (female)

E. Celebes (male)

Hapane (female)

S. Celebes

Sikkim

Sikkim

Indo-China (female)

Tonkin (female)

Hainan

Hong-Kong

Malacca (male)

Perak (female)

Lombok

Palawan

W. Sumatra

Sandakan

Ceram

Sumatra

W. Java

E. Java

Palawan

Jolo

Flores

Kalao

Ceram

Buru

Obi

Kiriwina

Halmaheira

Fergusson

Waigeu

Dorey

N. Borneo (male)

S. Borneo (female)

N. Borneo

?

Natuna 


\section{Nymphalidae (Continued)}

Cynthia erola javana

$$
\begin{gathered}
\text { " kohana } \\
\text { " kschattyia } \\
\text { arsinoe melena } \\
\text { " mesunia } \\
\text { " agatho } \\
\text { erota austrosundana } \\
\text { " arbotaeniaca } \\
\text { " meduca } \\
\text { " lucussa } \\
\text { " palawanica } \\
\text { " ladia } \\
\text { sapor laudo }
\end{gathered}
$$

Cupha lampetia mirona

myronides datos

lampetina

madestes zosima

decernia

couentia

fergussonia

erymanthis kangeana

erymanthis dohertyi

nagala

palawana

saturatior

maja

maeonides rovena

arias celebensis

" fedora

cacina

f. siberias

crameri keyana

Cirrochroa tyche philina rotundata $f$. caere

lesseta

kydrara

laudabilis

siamensis

thule $f$. massilia

omanrea f. martini

bajadetina

malaya natuna

calypso f. banona

clajia clajina

orissa orissides

satellita illergeta
W. Java

Batu

Bazelan

N. Irlande

Guadalcanar

N. Hannover

Lombok

Sumbawa

Maumerie

Goram

Palawan

Balabac

Peking

Choiseul

Buru

Batjan

Obi

Woodlark

Guaribari

Fergusson

Fergusson

Kangean

Engano

N. Borneo

Palawan

Lombok

Karvar

Celebes

Cent. Celebes

E. Celebes

Palawan

Mantanani

Key

N. Borneo

Siam (male)

Annam (female)

Hainan

Bazelan

Palawan

Siam

S. Celebes

Sumatra

Sumatra

Natuna

N. Borneo

Padang

N. Borneo

N. Borneo 
Nymphalidae (Continued)

Cirrochroa aolis f. stramentica semiranis minos paulowna

regina myra

n sophene

masica

princessa

Atella arcippe drepana

andamana

enganica

cervilides

floresiana

violeta

quinta

agoria

kinitus

alceppina

asinia

Issoria ejesta macromalayana

$$
\begin{aligned}
& \text { " " terrina } \\
& \text { ") " brixia } \\
& \text { " " abita } \\
& \text { " " " eda } \\
& \text { ". } \quad \text { scyllaria } \\
& \text { n orfeda } \\
& \text { offaka } \\
& \text { samoana } \\
& \text { buruana } \\
& \text { elvira } \\
& \text { edithea } \\
& \text { obiana }
\end{aligned}
$$

lerinos terpander natuensis

$$
\begin{aligned}
& \text { " } \\
& \text { " } \quad \begin{array}{l}
\text { porus } \\
\text { tethys udairs } \\
\text { taxiles helleri } \\
\text { " }
\end{array} \\
& \text { famphion } \\
& \text { clarissa maleana } \\
& \text { " dimaga } \\
& \text { " praestigiosa }
\end{aligned}
$$

Precis hedonia thero

$$
\begin{aligned}
& \text { numana } \\
& \text { apollonia } \\
& \text { teurnia } \\
& \text { siccata }
\end{aligned}
$$

Assam

S. Celebes

Batjan

Dutch New Guinea

New Guinea

Waigeu

Obi

W. Java

Andamans

Engano

Waigeu

Flores

Bazelan

Halmheira

Batjan

"Germ. "New Guinea

Obi

Wetter

Padang

Bazelan

Luzon

Babber

Wetter

Lifu

Key

Waigeu

Samoa

Buru

Batj an

Halmaheira

Obi

Banda

Natuna

Cochinchina

S. Celebes

Humbloldt Bay

Waigeu

Halmaheira

Siam

Sumatra (niale)

Penang (female)

W. Sumatra

N. Borneo

Banda

$\mathrm{Obi}$

Flores

Sula

Sikkim 


\section{Nymphalidae (Continued)}

Precis hedonia pluviatilis

timorensis cibota

valesca

orithyia hainensis

patenas

seleyra

sumatrana

orithya metion

leucasia

baweana

mevaria

kühni

minuscula

eutychia

halea

eucala f. phytites

erigone leucophora

himera

f. persiccata

lomonias aenaria

aenaria $f$. persicaria

vaisya

villida phylara

n procasa

atlites acera

armana battana

iphita tosca

$$
\text { cebara }
$$

Pyrameis dejeani sambaluna

indica buana

" nubicola.

atalanta rubria

Vanessa caschemirensis aesis polychlorus dilucidus

canace neojapanica

$$
\begin{aligned}
& \text { " dirilon } \\
& \text { " maniliani } \\
& \text { " javanica } \\
& \text { " ishima } \\
& \text { " siphnos }
\end{aligned}
$$

R. album Samaurai

Hypolimnas, alimene afra

obsolescens

talauta
Ceylan

Sumba

Alor

Hainan

Ceylan

Saleyr

Sumatra

Sumatra

N. Borneo

Luzon

Bawean

Lombock

Birnungka

Sumba

Wetter

Tenimber

Tonkin

Kiriwna

New Guinea

Java Emrid (male)

Bawean (female)

Formosa

Formora

Annam

Sumba

Tenimber

N. Celebes

S. Celebes

Sumatra

Sumba

Lombock

S. Celebes

Ceylan

Mexico (male)

N. Amer. (female)

Darjeeling

Saratow

Japan

Formosa

N. Borneo

W. Java

Ishigaki

Oshima (male)

Japan (female)

Japan

Kiriwina

Fergusson

Talaut

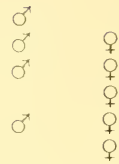

0

q

8

o

8

$0^{2}$

.




\section{Nymphalidae (Continued)}

Hypolimnas alimene lamina

$\begin{array}{ll}\text { " } & \text { duphridas } \\ \text { " } & \text { ysabella } \\ \text { " } & \text { libisonia } \\ \text { " } & \text { eligia } \\ \text { " } & \text { senia } \\ \text { " } & \text { senia f. diadema } \\ \text { " } & \text { remijia } \\ \text { " } & \text { pandana } \\ \text { " } & \text { saturnea } \\ \text { " } & \text { selina } \\ \text { " ciricta } & \text { bateia }\end{array}$

antilope truentus

" euvaristes

phalkes

stellate

mola

cjeuinitinus

f. sila maglovius

f. pseudopithoeka nysibtes

pithoeka $f$. illumanata

bolina $f$. luctuosa

f. chrysonicans

f. eulyxantha

lisianassa $f$. discalis

f. luctiosa

f. astra

philippines $f$. lictrix

philippines $f$. subucula

jolana

f. ornamentalis

f. subviolacea

f. pseudomorphus jaluita

micromalayana

pandarus $f$, triomphanus

deois deoina

saundersi jinia

deois obianus

anomala $f$. circonscripta

arnodi

palladius $f$. tydeina
Queensland

Shortland I

Ysabel

Milne Bay

Batjan

Buru

Buru

Obi

Banda

Waigeu

Dammer

Mafor

Yule 1.

Ishigaki

Bazelan (male)

Mindanao (female)

Talaut

N. Celebes

Kiriwina (male)

Fergusson (female)

Batjan (male)

Obi (female)

Amboina

Buru

Ceram

New Guinea

"Germ" New Guinea

Engano

Engano

Lombock

Saparua

Saparua

Lombock

Bazelan

Palawan

Jolo

Sikkim

Kashmir

NewGuinea

Jaluit

Babber

Saparua

Milne Bay

Wetter

Obi

Bawean

Sumbawa

Fergusson 


\section{Nymphalidae (Continued)}

Hypolimnas paleutes $f$. depunctata diomaea $f$. cerulans

Cyrestes thyodamas mabella

$$
\begin{aligned}
& \text { nivalis tonkiniana } \\
& \text { lutea doliones } \\
& \text { maenalis oebasius } \\
& \text { cocles cocleoides } \\
& \text { cassander archomenis } \\
& \text { paulinus waigeuensis } \\
& \text { periander enganicus } \\
& \text { " martinus } \\
& \text { " batinia }
\end{aligned}
$$

acilia bettina

$$
\begin{aligned}
& \text { sicca } \\
& \text { abisa } \\
& \text { dola } \\
& \text { gades } \\
& \text { tervisia } \\
& \text { bassara }
\end{aligned}
$$

Chersonesia rahria mandolina

$$
\text { sanna }
$$

Vanessa antilopa asopos

$$
\text { " yedanula }
$$

Doleschallia dascyllus phalinus anicotus eudascyllus

crameri lactearia servisina sinda sophilis

hexopthalmis ardys

varis
demades
domis
areus
kapaurensis
solus
gaius

noorna lyncurnion antinia

nacar trachelis scotinia

bisaotide orthorgonia celebensis sulaensis tenimbensis
Trobiandis

N. Celebes

Ishigaki (male)

Oshima (female)

Tonkin

Bali

Bazilan

Tonkin

Bazelan

Waigeu

Engano

Sumatra

Tonkin

Sula

Buru

Obi

Fergusson

Area (male)

Yule I. (female)

Dutch New Guinea

Hatley

Sula

Batu

Japan

Siao-Lou

Waigeu

Yule

New Guinea

New Guinea

Fergusson

Milne Bay

Halmaheira

?

Kiriwna

Milne Bay

Woodlark

Waigeu

Kapaur

Obi

Halmaheira

"Germ" New Guinea

Fergusson

Waigeu

Shortland I.

N. Ireland

N. Celebes

Sula

Tenimter

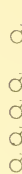

(

o

$\sigma^{x}$

o

ర大

0

0

$0^{x}$

б

o

9
+
+
+

o

0

$0^{x}$

q

q

0

$\sigma$

0 


\section{Nymphalidae (Continued)}

Doleschallia bisaotide

apameia

bisaltide tualensis

f. cingara
nasica
vomana
mariae
ermelinda
sulculus
scapus
continentalis
malabarica
andomanensis
ceylanica
siamensis
Borneensis
philippines

Yoma sabina vasilia
vasiki f. sabulosa
f. purpuria
f. foliaca

arjina $f$. taurusca

vestina

f. viruna

odilia

netonia

australis

Rhinopalpa polynice helionis

"f. Helma
acharis
birmana
callonis
tamora
famora f. necturnea
amoenis
cunis

Kallina inachis $\mathrm{f}$. marmorata

$$
\begin{aligned}
& \text { f. acerifolia } \\
& \text { f. uredinophora } \\
& \text { eucerca } \\
& \text { formosana } \\
& \text { siamensis }
\end{aligned}
$$

Halmaheira (male)

Batjan (female)

Obi

Key

Key

Waigeu

Viti Levu

Sumatra

Sumba

Bawean

Wetter (male)

Kisser (femaley

Sikkin

Malabar

Andamanes

Ceylan

Siam

N. Bornéo

Palawan (male)

Bazelan (female)

Batjan

Formosa

Alor

Alor

Milne Bay

Kapaur

"Germ" New Guinea

Fergusson (male)

Kiriwina (female)

Milne Bay

Queensland

N. Borneo

N. Borneo

Natuna

Lower Burma

Nias

Nias

Bazelan (male)

Mindanao (female)

Mindanao

Mindoro

S. Celebes

Tonkin

Assam

Assam

Liukiu (male)

Okinawa (female)

Formosa

Siam 


\section{Nymphalidae (Continued)}

Kallina limborgi tribonia

Symbrenthia hippocrus lucianus

formosana

sumatranus

marius

baliensis

niasicus

batuensis

lombokensis

sumbawensis

sperchius •

anna Semp.

galepsus

thimo

hypselis assama

circesia

ottilia
W. Sumatra

Malacca

Formosa

Sumatra

N. Borneo

Bali

Nias

Batu

Lombok

Sumbawa

Bazelan

Philippines

Mindoro

Luzon

Assam

Sumatra

Nias

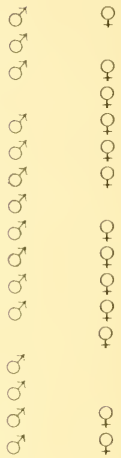

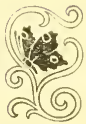




\section{NYMPHALIDAE (American)}

Prepona laertes pallantias ikarios

$$
\begin{aligned}
& \text { "f. autolycus } \\
& \text { penelope } \\
& \begin{array}{l}
\text { pallidior } \\
\text { f. antiklia }
\end{array}
\end{aligned}
$$

omphale abulonia

$$
\text { " octaria }
$$

dexamenes luktra

$$
\begin{array}{ll}
\text { " } & \text { krates } \\
\text { n } & \text { psocon }
\end{array}
$$

pylene proschion

$$
\text { " bahiana }
$$

chromus xenarchus

lycomedes scyrus

pheridamas phila

pheridamas attalis

chalciope domna

$$
\text { " chalcis }
$$

meander psudomeander

$$
\begin{array}{ll}
\text { " } & \text { negabates } \\
\text { " megacles } \\
\text { " magina } \\
\text { solon }
\end{array}
$$

antimache crassina

$$
\begin{array}{ll}
\text { " } & \text { ilmatar } \\
\text { " } & \text { insulicola } \\
\text { " } & \text { lyda } \\
\text { " } & \text { andicola } \\
& \text { gulina }
\end{array}
$$

demophon xiniatus

$$
\begin{array}{ll}
\text { " } & \begin{array}{l}
\text { pamenis } \\
\text { zoranthes } \\
\text { muson }
\end{array} \\
\text { " } & \text { ventralis }
\end{array}
$$

amphimachus magas

$\begin{array}{ll}\text { " } & \text { symaithus } \\ \text { " } & \text { cinctus } \\ \text { " } & \text { amphiktyon } \\ \text { n fruhstorferi Rober. }\end{array}$

Adelpha pseudomessana

$$
\text { lerna archidona }
$$

Adelpha issia lucas

$$
\text { naxia zyzia }
$$

$$
\text { " murica }
$$

Colombia

Surinam

Obidos

Bolivia

Bolivia

Paraguay

Bahia

Honduras

Bresil

Surinam

Matto Grosso

Rio Grande

Bahia

Peru

Perv

Minas Geraes (male)

Espiritu Santo (female)

Bolivia

Theresopolis

Paraguay

Rio Grande

Peru

Bresil

Matto Grosso

Theresopolis

Cuba

Trinidad

Haiti

Matto Grosso

Ecuador

Honduraz (male)

Costa Rica (female)

Bahia

Minas Geraes

Rio Grande

Colombia (male)

Ecuador (female)

Honduraz

Bolivia

Ecuador

Mexico

Honduraz

Bresil

Peru

Ecuador

Mexico

Matto Grosso

Chiriqui 


\section{Nymphalidae American (Continued)}

Adelpha naxia fundomia

$$
\begin{aligned}
& \text { phylaca phylacides } \\
& \text { " georgias } \\
& \text { abia mincia } \\
& \text { " rufilia } \\
& \text { calliphana } \\
& \text { thessaria cesilas } \\
& \text { gavina } \\
& \text { epizygis } \\
& \text { epona } \\
& \text { falcipennis perga } \\
& \text { iphicla exanima } \\
& \text { " gortyna } \\
& \text { " massilides } \\
& \text { " pharae } \\
& \text { "iphimedia } \\
& \text { " deceleia } \\
& \text { " gellia }
\end{aligned}
$$

basiloides caolia

thoasa cingaba

$$
\text { " manilia }
$$$$
\text { n zalma }
$$$$
\text { ") gerona }
$$

serpa damon

seriphia aequillia

$$
\text { " piono }
$$

celerio diademata

zea tarpeia

" serpentina

nea campeda

mythra patricia

cytherea marcia

$$
\begin{array}{ll}
\text { " } & \text { despoliata } \\
\text { " } & \text { tarratia } \\
\text { " } & \text { lanilla }
\end{array}
$$$$
\text { n heremia }
$$

aricia serenita

alala cora

$$
\begin{aligned}
& \text { "negrina } \\
& \text { " } \text { f. fillo } \\
& \text { n completa } \\
& \text { n } n \text { f. precaria } \\
& \text { nf. titia } \\
& \text { cora f. albifida } \\
& \text { ") f. privigna }
\end{aligned}
$$

Colombia

Peru

Matto Grosso

Bolivia

Paraguay

Rio Grande

Rio

Rio Grande

Blumenau

Rio Crande

Bahia

Blumenau

Venezuela

Colombia

Mexico

Peru

Cuba

Trinidad

Blumenau

Colombea

Matto Grosso

Bolivia

Peru

Paraguay

Blumenau (male)

Santa Catherina (female)

Colombia

Colombia

W. Mexico

Rio Grande

Santa Catherina

Colombia

Rio Grande

Honduraz

Colombia

Ecuador

Bolivia (male)

Peru (female)

Brésil

Peru

Peru

Bolivia

Argentine

Colombia

Colombia

Venezuela

?

Peru

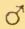

$\sigma^{x}$

$0^{x}$

o

0

o

$\sigma^{\pi}$

$0^{x}$

$0^{x}$

$\sigma^{x}$

б

$\pi$

o

o

0

$\sigma^{\pi}$

ox

o

$0^{\pi}$

$\sigma^{x}$

ర

$0^{x}$

o

व
व

$0^{x}$

$0^{\pi}$

o

$\sigma^{7}$

$0^{7}$

ox

$0^{x}$

$\stackrel{+}{q}$ 


\section{Nymphalidae American (Continued)}

Adelpha aethalia metane

" pollina

euboea mamaa

" fabricia

n hilarcia

phliassa implicata

" bartolme

plesaure heredia

" cerachates
$"$ sirona
" antoniae
jordani
cocala urracina
" didia
"riola
" siminia

valentina thyrea

" ophidusa

n ozolis

velia veliada

boreas verenda

irma

" nadja

irmina vorhelnina

boreas tizonides

sichaeus privata

" irisa

n leucophebia

salmoneus emilia

leucopthalma pantarheia

$$
\text { n tegeates }
$$

justina praevalida

" justinella

zina leucacantha

olynthia olynthina

" theaena

" zopyra

zina restricta

boeotia fidonia

fulvia

uta

erotia faebina

" f. tyrea

" permagna

" delphicola f. predura

" ophellas

1) improvida
Peru

French Guiyana

Matto Grosso

Colombia

Peru

Peru

Matto Grosso

Brésil

Matto Grosso

Bolivia

Blumenau

Peru

Upper Amazonas

Brésil

Brésil

Brésil

Bolivia

Ecuador

Peru

Blumenau

Peru

Peru

Bolivia

Bolivia

Colombia

Ecuador

Peru

Bolivia

Colombia

Colombia

Colombia

Peru

Venezuela

Colombia

Ecuador

Peru

Peru

Cananche (cundinamarca)

Bresil

Bolivia

Bolivia

Peru

Colombia

Peru (male)

French Guiana (female)

Para

Paraguay (male)

Mexico (male)

Bahia

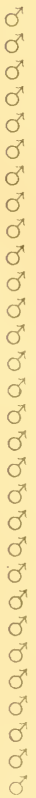

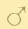

$\sigma$

$\sigma$

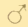

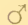

8

0

$\sigma$

o

$0^{x}$

$\pi$

o

8 


\section{Nymphalidae American (Continued)}

Adelpha isis divina

$$
\begin{aligned}
& \text { " pseudagrias } \\
& \text { " panthalis } \\
& \text { lara manias } \\
& \text { epione maina } \\
& \text { " agilla } \\
& \text { eponina volupus } \\
& \text { attica carmela } \\
& \text { " cerita } \\
& \text { melona leucovina } \\
& \text { arete pseudarete }
\end{aligned}
$$

Zaretes pseuditys

$$
\begin{aligned}
& \text { russeus } \\
& \text { isidora vulpina }
\end{aligned}
$$

f. bisaltide

f. polcarea

lutulenta

vulpercula

Didonis biblis sisygambis

$$
\text { " " nectanabis }
$$

Anaea chaeronea corita

Acherontia blomfildia datis

Napeocles jucunda ? dumnoria

Cybdelis phaesyle lysa

$$
\text { " mnasyllus sophistes }
$$

Catonephele batesi aestherica

$$
\text { obrinus faventia }
$$

Victorina steneles $\mathrm{f}$. pallida

$$
\begin{aligned}
& \text { " meridionalis } \\
& \text { ") sophene }
\end{aligned}
$$

Precis lavinia michaelesi

Amphirene epaphus ficianus

Pyrrhogyra edocla anthele

$$
\begin{array}{ccc}
n & \text { f. athene } \\
n & n & \text { aenaria } \\
n & \text { neaerea ollius } \\
n & n & \text { argina } \\
n & \text { n } & \text { kheili } \\
n & \text { crameri hagnodoris } \\
& \text { mantara } \\
n & \text { nasica seitzi } \\
n & \text { n olivenca }
\end{array}
$$

Peria livides asticta

n livia infirma

" " exilita

Eunica maevis aesochrion
Brésil

Blumenau

Brésil

Peru

Brésil

Colombia

Colombia

Colombia

Bolivia

?

?

Brésil

Colombia

Paraguay

Obidos

Obidos

Mexico

Bahia

Paraguay

Rio Grande

Brésil

W. Mexico

Matto Grosso

Peru

Bolivia

Colombia

Surinam

Matto Grosso

Mexico

Rio Grande

Ecuador

Porto Rico

Peru

Ecuador

Colombia

Honduraz

Honduraz

Upper Amazonas

Colombia

Upper Amazonas

Upper Amazonas (male)

Colombia (female)

Bolivia

Upper Amazonas

Upper Amazonas

Peru

Bahia

Paraguay

Paraguay 


\section{Nymphalidae American (Continued)}

Eunica margarita ebuonea

evelina alycia

bechina chorienes

cinara oreandra

celma celmina

tithonia intricata

cydonia poppacana

" ma-soandra

pomona pompata

norica occia

eurota theophania

" dymanes

alcymena irma

cabira gloriosa

cabira editha

tatila bellaria

marsalia fasula

Chlorippe beckeri floris

zalmuna paulistuna

kallina bertila

" zalunga

vacuna fluibunda

elis farge

" fabaris

pavon mellinia

" inumbratus

cyane phalysia

lavinia ornata

seraphina hippomanes

selina murorina

$$
\text { " modica }
$$

laure nitoris

$$
\begin{array}{ll}
\text { " } & \text { myia } \\
\text { " mima } & \text { majugena }
\end{array}
$$

Peridromia arete ortygia

$$
\begin{aligned}
& \text { " chaironia } \\
& \text { arethusa palliolata } \\
& \text { saurites } \\
& \text { amphinome morsina } \\
& " n \text { fumasa } \\
& n \quad \text { aegina }
\end{aligned}
$$

Peridromia amphinome sairis arinome obnubila

$$
\text { n }
$$$$
\text { n }
$$

sterope
Brésil

Upper Amazonas

Brésil

Peru

Bolivia

Brésil

Surinam

Upper Amazonas

Colombia

Peru

Bresil

Blumenau (male)

? (female)

Peru

Peru

Bolivia

Brésil

Upper Amazonas

Peru

Brésil

Paraguay

Bahia

Paraguay

Colombia

Bolivia

Mexico

Paraguay

Ecuador

Peru

Peru

Rio Grande

Brésil

Brésil

Paraguay

Colombia

Colombia

Honduraz

Bahia

Bolivia

Paraguay

Obidos

Honduraz

Colombia

Rio Dagua

Bahia (male)

Espirato Santo (female)

Bolivia

Brésil

Obidos
0
0
0
0
0
0
0
0
0
0
0
0
0
0
0
0
0
0
0
0
0
0
0
0

q

Q

q

q

$0^{2}$

$\sigma^{\pi}$

$0^{x}$

$\sigma^{x}$

o

$0^{x}$

ot

$\sigma^{x}$

$0^{x}$

$\sigma^{x}$

0

$\sigma^{\prime \prime}$

$\sigma^{x}$

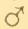

q

q

ㅇ

Q

ఠ

$\sigma^{x}$

o

$\sigma^{x}$

$\sigma^{\pi}$

$0^{x}$

Q 


\section{Nymphalidae American (Continued)}

Peridromia arinome ariensis

Ageronia ? chloerhoea

$$
\text { chloe obidona }
$$

" momia

" xenia

1) nais

ipthime aternia

" itania

") gervasia

epinome aldrina

„ florentia

fornàx fornacalia

guatamalena marmarice elata

mandra gora

feronia obumbrata

" insularis

" nobilita

) farinulentá

ferox gudula

" sedalis

" fumdaria

1) icilia

" sellasia

1) atinia

"sabatias

maina

fictitia

glauconome megala

$$
\begin{aligned}
& \text { " honorina } \\
& \text { " " f. julitta }
\end{aligned}
$$

Temenis laothoe violetta

$$
\begin{aligned}
& \text { " " hübneri } \\
& \text { " " bahiana } \\
& \text { " " hondurensis } \\
& \text { " " santina } \\
& \text { " } \quad \text { f. ottunus } \\
& \text { " " pseudolaothoe } \\
& \text { " flavilla lunigera }
\end{aligned}
$$

Anartia almathea thyannis

$$
\text { ") sticheli }
$$

" fatima mirus

" venusta

n jatrophae leutiprota

Anartiella lytrea eurytis

Megalura coresia dentigera
Colombia

Minas Geraes

Obidos

Bolivia

E. Bolivia

Tarapoto

Bolivia

Matto Grosso

Brézil

Bolivia

Bahia

Mexico (male)

Guatemala (female)

Mexico

Brézil

Brézil

?

Trinidad

Venezuela

Honduraz (male)

Mexico (female)

Texas

Mexico

Honduraz

Trinidad

Paraguay

Brezil

Matto Grosso

Peru

Mexico

S. Mexico

Yucatan

Yucatan

Rio Uaupes

Minas Geraes

Bahia

Horduraz

Brezil

Surinam

Obidos

Paraguay

Paraguay

Bolivia

Paraguay

Texas (male)

Guatemala (female)

Honduraz

Cuba

Peru (male)

Colombia (female)

0
0
0
0
0
0
0
0
0
0
0
0
0

ơ

o

0

రั

$\pi$

o

o

ช'

$\sigma^{x}$

రా

वै

$\sigma^{x}$

$\sigma^{x}$

o

$\sigma^{\pi}$

on

$\sigma^{x}$

Q

$\sigma^{x}$

0

$0^{x}$

0

o

ot

$0^{x}$

o'

$0^{x}$

0

$\sigma^{x}$

ช

$\sigma^{x}$

0

P

Q

P

P 


\section{Nymphalidae American (Continued)}

Megalura berania crassilinolates

" iole argentina

" corinna lazulina

Coloenis phaetusa metena

Metamorpha dido diatonia

$$
\text { " } \quad \text { pygmalion }
$$

Panacea prola prolifica

") amazonica

hypochlora chrysocantha

Catagramma atacama manosa

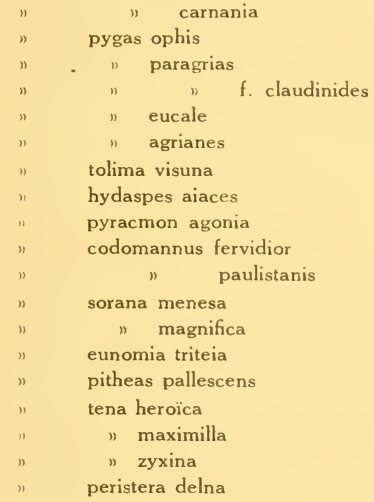

Callicore marchalli agonalia

$$
\begin{gathered}
\text { " aiakides } \\
\text { clymena osoria } \\
" \text { tyche } \\
" \quad \text { asterites } \\
\text { candrena teana } \\
\text { bifasciata } \\
\text { gabaza violanta } \\
\text { elmina zabira } \\
\text { vaninka philiatra } \\
\text { alicia paralicia }
\end{gathered}
$$

Perisama morona xynites carbinia thryoessa oppelii aisepus humboldti vestina

$$
\text { " exuberans }
$$

diotima demata

$$
\text { " zyxata }
$$

Orophila cecidas yurapa

$$
\text { 1) cecidina }
$$

Rio Uaupes

Argentina

Peru

Paraguay

Honduraz

Obidas

Ecuador

Amazones

Ecuador

Colombia

Peru

Bahia

Paraguay

Paraguay

Blumenau

Rio Grande

Costa Rica

Brezil

Brezil

Bolivia

Brezil

Bahia

Peru

Ecuador

Venezuela

Mexico

Brezil

Venezuela

Peru

Venezuela

Ecuador

Guatemala

Ecuador

Paraguay

Rio Grande

Colombia

Venezuela

Minas Geraes

Bolivia

Peru

Peru

Peru

Venezuela

Venezuela

Colombia

Ecuador

Peru

Peru

Peru $\sigma^{x}$
$0^{x}$
$0^{x}$
$0^{x}$

$\sigma^{x}$

o

ठ

万

o

$\sigma^{x}$

$0^{x}$

$\sigma^{x}$

$\sigma^{x}$

万'

б

o

$0^{x}$

ర

$\sigma^{x}$

$\sigma^{x}$

$\sigma^{x}$

$0^{x}$

ఠ

$\sigma^{x}$

ऽ

0

$0^{x}$

万

$\sigma^{x}$

万’

8

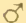

o

$\sigma^{x}$

$0^{x}$

$\sigma^{x}$

o

0

$0^{x}$

$0^{x}$

o

o

$0^{x}$

o

o 


\section{AMATHUSIIDAE}

Taenaris artemis xenada

$$
\begin{aligned}
& \text { " celsa } \\
& \text { " f. eleusina } \\
& \text { "f. lacrimans } \\
& \text { ziada } \\
& \text { " sticheli } \\
& \text { f. limbata } \\
& \text { f. artemides } \\
& \text { " pandemos } \\
& \text { gorgo danalis } \\
& \text { " meras } \\
& \text { gorgophone f. simplex } \\
& \text { " } \\
& \text { bioculatus charonides } \\
& \text { macrops macropina } \\
& \text { " ternatus } \\
& \text { rothschildi merana } \\
& \text { " f. pelagia } \\
& \text { wahnesi venaria }
\end{aligned}
$$

f. candida

f. tainides

f. hadina

onesemides

myops praxades

myops fergussonia

dioptrica wattina

amitaba

licinia

dimona offaka

kapaura

sophaineta

thaeina

zaitha

dimonata Stich.

sorronga

arceia

domitilla agrippa

onolaus rebeli

"f. auriflua

f. sidus Stich.

diana diadema

") leto

selenides attina

attina $f$. delunata

catops galaecia

y turdula
Dutch New Guinea

Salawatti

Dutch New Guinea

Dutch New Guinea

Misol

Milne Bay

New Guinea

New Guinea

Buru

Ceram

Waigeu

Dutch New Guinea

"Germ n. New Guinea

Germ ". New Guinea

Aroa (Brit. New Guinea)

Obi

Ternate

Dutch New Guinea

Dutch New Guinea

Salawatti (male)

Sorong (female)

New Guinea

"Germ ". New Guinea

New Guinea

Dutch New Guinea

Salawatti

Fergusson

Salawatti

Waigeu

Dutch New Guinea

Waigeu

Dutch New Guinea

Yule I.

"Germ ". New Guinea

Dutch New Guinea

Salawatti

Dutch New Guinea

New Guinea

$\mathrm{Obi}$

"Germ ". New Guinea

New Guinea

"Germ ". New Guinea

$\mathrm{Obi}$

Halmaheira

Waigeu

Waigeu

Dutch New Guinea

Yule I.

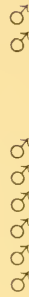

$0^{x}$
$0^{x}$
$0^{x}$
$0^{x}$
$0^{x}$
0

q

Q

$\sigma^{x}$
$0^{x}$

+
+
+
+
+

$\pi$
0

0
0

$q$
0
+
$q$

రా

0

o

万'

$\sigma^{x}$

o

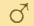

$\sigma^{x}$

o

o

o

$\sigma^{x}$

o

ox

ot

$\sigma^{x}$

Q

q

q

t

?

o

$0^{x}$

ot

$Q$
+
+
+
+

q

P

ช

웅 


\section{Amathusiidae (Continued)}

Taenaris catops mylacchoides

appina
westwoodi f. unipupillata
luna Stich.
laretta
jathrippa
kajuna
catanea
jobina

Zeuxidia doubledayi nicevillei

" luxeri luculenta Stich.

Amathuxidia plateni suprema

Amathusia phidippus dilutus

f. chiadesma

dilutus $f$. arrenopia

eutropius $\hat{f}$. aretracta

lombokiana

niasana

celebensis

adustalus

chersias

frederici

Pseudamathusia niasana

$$
\begin{aligned}
& \text { virgata thoanthea } \\
& \text { ochraceofusca gabriela } \\
& \text { perakana natuna } \\
& \text { incisa staudingeri Rôber } \\
& \text { orasis }
\end{aligned}
$$

Clerome aerope excelsa menado $f$. zenica surana

phaon ikonion eumaeus niarum borneensis niasana arcesilaus samathi

Aemona lena Atkins.

Thaumantis odana depupillata

$$
\begin{gathered}
\text { " pishuna } \\
\text { " pannvila } \\
\text { noureddin chatra } \\
\text { " sigirya } \\
\text { lucipor candika }
\end{gathered}
$$

Xanthotaenia busiris sadja

Discophora lepida ceylonica

$$
\text { tullia hainensis }
$$

n

$$
\text { " symphrania }
$$

Collingwood Bay

Milne Bay

New Guinea

"Germ ".New Guinea

Dutch New Guinea

Salawatti

Dutch New Guinea

Dutch New Guinea

Jobi

W. Sumatra

W. Sumatra

Sula

N. Borneo

S. E. Berneo

S. Borneo

Sumatra

Lombok

Nias

N. Celebes

Siam

Malacca

Tenasserim

Nias

N. Celebes

S. E. Borneo

Natuna

S. E. Borneo

Bawean

Lombok

Tonkin

Cent. Celebes

Sula

?

Dran (?) Annam

Natuna

Nias

Batu

Tenasserim

?

Malacca

N. Borneo

N. Borneo

Sumatra

Sumatra

Sumatra

Ceylan

Hainan

Perak 


\section{Amathusiidae (Continued)}

Discophora necho helvidius mindorana philippines odorata engamon cycnis verbanus euthymius duranus
S. Borneo
Mindoro
Bazilan
Palawan
Malacca
Bhutan
Padang

\section{BRASSOLIDAE}

Dynastor darius mardonius faenius

Caligo cileus philinos phorbas Rôber. philademus oedippus Stich. prometheus atlas Rôber. idomeneus ibycus idomenides

memnon menes 1) anaximander teucer obidonus phoroneus nubilus eurylochus sulanus

$$
\text { " " pallidus }
$$

ilioneus pampeiro " praxiodus

Opsiphanes cassiae pudicus

\begin{tabular}{|c|c|c|}
\hline & "le & lucullus \\
\hline & $"$ & dercentius \\
\hline " & tamarind & di pseudocassiae \\
\hline " & invirae $r$ & remoliatus \\
\hline ") & $n$ & pseudophilon \\
\hline "n & $"$ & ? reluceus \\
\hline ") & $n$ & ampliticatus \\
\hline " & $"$ & isagoras \\
\hline " & $n$ & agasthenes \\
\hline & $n$ & f. vectitas \\
\hline & quiteria & philon \\
\hline " & $n$ & obidonos \\
\hline " & " & mylasa \\
\hline v & 1 & ? ovebios \\
\hline
\end{tabular}

Paraguay

Rio Grande

Bolivia

Ecuador

Colombia

Colombia

Ecuador

Minas Geraes

Peru

Chiriqui

Colombia

Obidos

Rio Uaupes

?

Honduraz (male)

Chiriqui (female)

Bolivia

Paraguay

Peru

Bahia

Brezil

Minas Geraes

Manaos

Brezil .

Brezil

Honduras

Paraguay

Bolivia

Peru

Peru

Brezil

Obidos

Rio Uaupes

Paraguay

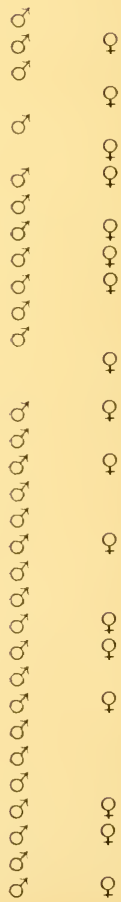




\section{Brassolidae (Continued)}

Selenophanes cassiope theognis " amphire spintarus

Catoblebia xanthicles sosigenes

Catoblebia xanthicles sosigenes ciparissa berycynthina miditaenia berecynthia $f$. latitaenia
Matto Grosso

Brezil

Brezil

Cayenne

Colombia

Rio

Surinam
Morpho laertes eutropius

$$
\begin{array}{cl}
\text { catenareus } & f . \text { marmorata } \\
\text { " } & \text { f. nigrescens } \\
\text { " } & \text { defigurata } \\
\text { " } & \text { argentinus }
\end{array}
$$

theseus yaritanas

theseus aratos

achilles trojana Rôber.

$$
\text { ") f. mystica }
$$

phokylides

agamedes

unicans

amazonicus

pindarus

bahiana

peleides cortane

maculata Rôbes.

pudicis

zela

hyacinthius $f$. erospitaenia

hector subfasciata Rôber.

achillaena paulista

deidamia erica

granadensis lycanor

patroclus rugitaeniatus
Brezil

Brezil

Brezil

Brezil

Argentina

Venezuela

Honduras

Paraguay

Paraguay

Paraguay

Bolivia

Amazones

Rio Uaupes

Obidas

Matto Grosso

Bahia

Colombia

Ecuador

Nicaragua

Mexico

Honduras

Peru

Brezil

Obidas

Ecuador

N. W. Ecuador 


\section{SATYRIDAE (Palaearctic)}

Melanargia galathea tenebrosa

\begin{tabular}{|c|c|c|}
\hline " & $"$ & syntelia \\
\hline " & $n$ & auricoma \\
\hline " & $"$ & pygmaea \\
\hline$"$ & $n$ & nereus \\
\hline$n$ & $"$ & helalla \\
\hline$"$ & $"$ & doris \\
\hline$n$ & $n$ & sciritis \\
\hline$n$ & $n$ & galinthias \\
\hline$"$ & $"$ & gattinara \\
\hline$"$ & $n$ & donsa \\
\hline " & $"$ & conbona \\
\hline$n$ & $"$. & akis \\
\hline$"$ & $"$ & symaithis \\
\hline " & $"$ & sakaria \\
\hline " & $"$ & eudaemовіа \\
\hline " & $"$ & florina \\
\hline " & halimede & pasiteles \\
\hline " & japyga in & iglada \\
\hline$"$ & " ja & lemus \\
\hline 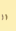 & larissa lyc & \\
\hline
\end{tabular}

Epinephele rhamnusia najera

calsona
" nikokles
" $\quad$ salona
onus decolorata
aon fluminius
" okeanina
" macropthalma
" lycosura
" ephisius

Coenonympha iphis anaxarete typhon thymoites " ferinana " pamphylus orantia

Aphantopus hyperantus rufilins

Erebia nerine neobica Turati.

$$
\begin{aligned}
& \text { " " turatii } \\
& \text { gyrtona } \\
& \text { triglites } \\
& \text { vorbrodti } \\
& \text { afer xianta } \\
& \text { afer fidena } \\
& \text { " bardines } \\
& \text { melas } f \text {. carbonaria }
\end{aligned}
$$

Krain

Dalmatia

Galicia

Savoy

Wallis (male)

Martigny (female)

Oberdorf

Digne

Rom.

Neapol

Castile

Tiflis

Kertsch

Alp. Mmes.

Saratow

Lana

Sicily

Wallis

China

Castile

Kashgar

Ulbrich

Andalusia

Tian-Shan

Iserle

Wetti (Prenj.)

Andalusia

Wallis

Salève

Castile

Alp. Mmes.

Courmajeur

Alp. Mmes.

Martigny

Kashgar

Kashgar

Klausen (male)

Lama (female)

Primiero

Krain

?

Tessin

Engadine

Caucasus

Transcaspia

Altai

Ungarn

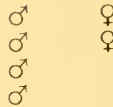

$\stackrel{+}{q}$

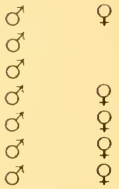

$\sigma^{x}$

$0^{x}$

$0^{x}$

?

P

Q

$0^{x}$

$\sigma^{x}$

$\sigma^{x}$

$\sigma^{x}$

б

$\sigma^{x}$

o

$\sigma^{x}$

ó

o

$0^{x}$

o

o 
Satyridae (Continued)

Erebia melas nanos

$$
\begin{aligned}
& \text { " aoris } \\
& \text { pyrene charia } \\
& \text { " praerutilia } \\
& \text { " cubei } \\
& \text { " calaritas } \\
& \text { " jerasa } \\
& \text { " castiliana } \\
& \text { " aeme mythia } \\
& \text { aeme clisura } \\
& \text { noctua } \\
& \text { alecto anthracites } \\
& \text { " } \quad \text { velocissima aurbo } \\
& \text { " } \\
& \text { " anteborus } \\
& \text { medusa charila } \\
& \text { " meisneri } \\
& \text { " } \quad \text { narona } \\
& \text { " euphrasia } \\
& \text { euyale etobyma } \\
& \text { " antevortes } \\
& \text { syrmia }
\end{aligned}
$$

ligea urkostrate

$$
\text { " carthusia f. norum }
$$

$$
\begin{array}{ll}
\text { n } & \text { siscia } \\
\text { " } & \text { permagna } \\
\text { " } & \text { postvorta } \\
\text { n } & \text { pellea }
\end{array}
$$$$
\text { pharte eupompa }
$$$$
\text { 1) flaccilla }
$$$$
\text { " pellena }
$$$$
\text { 1) thymias }
$$

pyrene laconia

" posidonia

11 freyeri

aethiops dernfates

$$
\text { " salaria }
$$

ceto crobyle

$$
\text { " cetra }
$$

Krain

Bulgaria

Velebit Ostarie

Salève

Dingy

Alp. Mmes.

Digne

Jerva

Castile

Bad Oberdors

Jegernsee

North Tirol (male)

Campolungo (female)

Schnastal

Switz

Ortler (male)

Teriolis (female)

Susten (male)

Lenk (female)

Versoix

Schwarzwald (male)

Neuveville (female)

Bosnia

Bulgaria

Campement

Alp. Mmes.

Pyrénées

Wetti (Irebevjé)

Ampezzo

Col D'annes (male)

Chartr. (female)

Appenines

Alp. Mmes.

Wetti (Hodya)

Altai

Schliersee

$\checkmark$ al Blenio (male)

San Bernhardino (female)

Platte (male)

Glockner (female)

La Grâve

N. Vogesen

Schwarzwald

Bad Oberdorf

Primiero

Klausen

?

Alp. Mmes. 
Satyridae (Continued)

Erebia ceto rhodocleia

$$
\begin{aligned}
& \text { pronoe tarcenta } \\
& \text { n zynuta } \\
& \text { manto erina } \\
& \text { melampus audineus } \\
& \text { tigranes } \\
& \text { cercida momos } \\
& \text { generosa } \\
& \text { pronoe psathura } \\
& \text { goante homole }
\end{aligned}
$$

Epinephele pasiphae aurycles janira phormia

Pararge aegeria eudokia eleganta egestas camoera

hiera calidia

$$
\text { " sestia }
$$$$
\text { " falcidia }
$$

semele f. jubaris

$$
\begin{aligned}
& \text { " subcinericia } \\
& \text { " terea }
\end{aligned}
$$

maera silymbria praegrandis ordona

arethusa galatea

$$
\begin{array}{ll}
\text { " } & \text { ganda } \\
\text { " } & \text { alpheiros } \\
\text { n } & \text { segusiana }
\end{array}
$$

semele cadmus

$$
\text { " senthes }
$$

deidamia interrupta

$$
\text { " thyrias }
$$

Satyrus actaea castiliana

$$
\begin{aligned}
& \text { " } \text { podareina } \\
& \text { " } \text { gavina } \\
& \text { ferula serva } \\
& \text { " penketia } \\
& \text { " molykreia }
\end{aligned}
$$

Satyrus dryas tassile

autonoe ordomenus thermus

geyeri aristomicus regeli minuitianus anthelea schawerdae heydenreichi hegesander
Klausen (male)

Penegal (female)

Ampezzo

Bulgaria

Allgau

Simplon

Chamonix

Klausen

Fusio

Generoso

Pizzo Leone

Digne

Castile

Lana

Algerie

Neuveville

Dalmatia

Lana

Salève

Altai

Kentei

Konigsberg

Castile

Digne

Dalmatia

Saratow

Bayern

Castile

Auzay (Vendée)

Urals

Piemont

Klausen

Griechenland

Japan

Tsingtau

Castile

Andalusia

Alp. Mmes.

Dalmatia

Griechenland

Tian-Shan

Worishofen

Tian-Shan

Turkestan

?

Altai Mont

Herzogovina

Tian-Shan

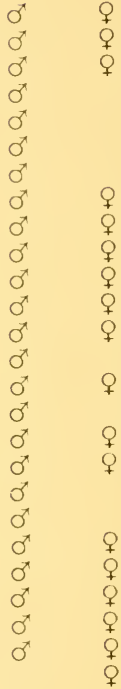

ช

o

ठ

б

ช

o

$0^{x}$

o

o

o

o 


\section{Satyridae (Continued)}

Satyrus ? miriszechi esceuilinius briseis cataca

$$
\begin{array}{cl}
\text { " } & \text { hyrnessus } \\
\text { " } & \text { emilianus } \\
\text { " } & \text { turatii } \\
\text { " } & \text { saga } \\
\text { circe venusta } \\
\text { " venifica } \\
\text { " praestigiata } \\
\text { " paralenca } \\
\text { " illecebra } \\
\text { " naga } \\
\text { " panonnia } \\
\text { " teulada } \\
\\
\text { " briseis diminuta }
\end{array}
$$$$
\text { schrenki damontas }
$$$$
\text { " menelacas }
$$$$
\text { achine mendelensis Lawe }
$$$$
\text { alcyone vivila }
$$$$
\text { vipsaria }
$$$$
\text { fagi genava }
$$$$
\text { ") albifera }
$$$$
\text { orphnia }
$$$$
\text { aturia }
$$$$
\text { tetrica }
$$$$
\text { syriaca cerrula }
$$$$
\text { ") attikana }
$$

Altai Mont

Castile

Saratow

Italy

Sicily

Dalmatia

Caucasus

Var

Florence

Castile

Ungarn

Auzay (Vendee)

Ultrich

Sardinia (male)

Corsica (female)

Kals (?) Bosnia

Sze-Chuan

Sapporo

Salève (male)

Savoy (female)

Savoy

Castile

Wallis

Waidbruck (male)

Klausen (female)

Piemont (male)

Florence (female)

Alp. Mmes.

Saratow

Dalmatia

Griechenland 
INDO-MALAYAN

Mycalesis anaxias bisaltia

$$
\begin{gathered}
\text { " aemate } \\
\text { adamsoni definiens } \\
\text { francisca ulia } \\
\text { " formosana } \\
" \text { atia } \\
\text { perdiccas } \\
\text { f. depupillata } \\
\text { gotama nanda } \\
\text { " varcella } \\
\text { duponcheli roonia } \\
\text { " scriptus } \\
\text { " maforica } \\
\text { kapaura }
\end{gathered}
$$

delicata

perseis intricatus

perseis typhlus

horsfieldi pantaka

$$
\text { ptyleus }
$$

niasana

tessinus

enganicus

lucinoe

lermana

lucianus

deria

avarga

mineus macromalayana

$$
\text { " nevayana }
$$

fuscum musculus

$$
\begin{aligned}
& \text { f. macularia } \\
& \text { " adonstata }
\end{aligned}
$$

\begin{tabular}{|c|c|}
\hline Assam & $\sigma^{x}$ \\
\hline ? & $\sigma^{x}$ \\
\hline Tonkin & $\sigma^{7}$ \\
\hline Tonkin & $0^{x}$ \\
\hline Formosa & \\
\hline Tenasserim & $\sigma^{x}$ \\
\hline Annam & \\
\hline Japan & 0 \\
\hline Japan & $\sigma^{7}$ \\
\hline Formosa & $\sigma^{x}$ \\
\hline Japan & $\sigma^{x}$ \\
\hline Roon & $\sigma^{7}$ \\
\hline Mafor & $\sigma^{x}$ \\
\hline Kapaur & $\sigma^{x}$ \\
\hline Lombok & $\sigma^{x}$ \\
\hline Lombok & $\sigma^{x}$ \\
\hline Malabar & $\sigma^{7}$ \\
\hline Formosa & $\sigma^{7}$ \\
\hline Saleyer & $0^{x}$ \\
\hline Nias & $\sigma^{7}$ \\
\hline N. Celebes & $0^{x}$ \\
\hline Engano & $\sigma^{\prime}$ \\
\hline Palawan & $\sigma^{7}$ \\
\hline N. E. Sumatra & 0 \\
\hline Annam & $0^{x}$ \\
\hline Bali (male) & \\
\hline S. Java (female) & ఠ \\
\hline Palawan & $0^{x}$ \\
\hline Sumatra & $\sigma^{7}$ \\
\hline S. Celebes & $\sigma^{x}$ \\
\hline Nias & $\sigma^{7}$ \\
\hline Sumatra & \\
\hline N. Borneo & $\sigma^{\prime}$ \\
\hline Bazilan & o \\
\hline Sula Besi & $0^{\prime}$ \\
\hline Mindanao & $\sigma^{x}$ \\
\hline N. Borneo & $\sigma^{\prime}$ \\
\hline Sumatra & $\sigma^{x}$ \\
\hline Lombok & $\sigma^{x}$ \\
\hline S. Celebes & 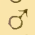 \\
\hline N. Borneo & $\sigma^{x}$ \\
\hline Formosa & \\
\hline Jolo & $\sigma^{\prime}$ \\
\hline Palawan & \\
\hline Nias & $\sigma^{x}$ \\
\hline S. Borneo & व' \\
\hline $\begin{array}{l}\text { N. Celebes (male) } \\
\text { S. Celebes (female) }\end{array}$ & $\pi$ \\
\hline
\end{tabular}

micromede

janardana besina

$$
\text { virella }
$$

baluna

saggitigera

sapitana

operculus

anapita fiorenta

periscelis

ita jolana

"palawensis

orse is orsina

") borneensis

itys remulina 


\section{Indo-Malayan (Continued)}

Mycalesis durga jobina

" enhoredata

sirius antevanis

canicula

terminus atropates

terminulus

wakolo

ternatensis

pseudasophis

anteros

arabella

bilineata

discobelus

dexamenus transiens

ahaaseai unipupillata

pandaca deianirina

celebina inga

") erna .

tagara palawana

1) mindorana

n semirasa

tilmara

visaya samina

mnasicles perna

mucia verena

" f. valde

" praxedis

1) febronia

v etha

evara

n evalida

shiva arima

" sastra

1) gopaka

aethiops copiosa 1) lormides

elia thelphila

mamerta f. Mausonia 1) annamitica

malsarida inopia

visala neovisala

nicotia mudgara

suaveolens kagina

mestra vetus

medus f. turbata

" zipoetina

1) buruana

jopas mendice
Jobi

New Guinea

New Guinea

Buru

Dutch New Guinea

Waigeu

Buru

Ternata

Batjan

Halmaheira

Waigeu

Milne Bay

Aroa

Cent Celebes

E. Celebes

N. Celebes

Sula Besi

S. Celebes

Palawan

Mindoro

Bazilan

Sangir

Mindoro

Tonkin

- Germ n. New Guinea

New Guinea

Dutch New Guinea

Jobi

Fergusson

Milne Bay

Germ n. New Guinea

Aru

New Guinea

Waigeu

Germ ". New Guinea

Mafor

Germ n. New Guinea

Tonkin

Annam

Tonkin

Tonkin

Tenasserim

Formosa

Bhutan

Nicobars

N. E. Sumatra

Buru

S. C elebes 


\section{Indo-Malayan (Continued)}

Mycalesis jopas paupercala

mystes tumicola
dohertyi excelsior
mara

duponchelli urbonia

$$
\begin{array}{ll}
n & \text { andersoni } \\
\text { n } & \text { eudromia }
\end{array}
$$

Zethera pimplea gadrosia

$$
\text { " musa mixta }
$$

Ptychandra lorkuni caerulans

$$
\begin{array}{lcl}
\text { " } & \text { " mindanaensis } \\
\text { " } & \multicolumn{3}{c}{\text { schonbergil } f \text {. hebetatrix }}
\end{array}
$$

Anadebis batinara

Coelites nethis sylvarum

Aulocara swaha garuna

$$
\begin{array}{lll}
n & \text { " } & \text { tellula } \\
& \text { mardana }
\end{array}
$$

Orinama damaris harmotus

Callerebia nirmana scandina

Lethe noclides

$$
\begin{aligned}
& \text { insularis } \\
& \text { sidonis gelduba } \\
& \text { ocellata bojonia } \\
& \text { syrcis dicimaga } \\
& \text { verma sintica } \\
& \text { " cintamani } \\
& \text { " stenopa } \\
& \text { " sataramus }
\end{aligned}
$$

Lethe insana formosana

diana celga

sycelis vanelia

pegiona farissa

mataja

latiaris perimele

christophi hanako

kansa zengitana

" vaga

vindhya ladestes

cerama Shelf.

europa miladana

ragalba

gada

mudgara

pavida
Bangkei (male)

Sula (male)

Siam

N. Borneo

Formosa

Waigeu

Kiriwina

Fergusson

?

Bazilan

Mindoro

Luzon

Mindanao

Bazilan

Mindoro

Tonkin

Tonkin

India

India

India

Tenasserim

India

Formosa

Formosa

Sikkim

Formosa

Tonkin

Sikkim

Formosa

Tonkin (male)

Tenasserim (female)

Tze-Chuan

Formosa

Japan

Japan

Upper Burma

Formosa

Tenasserim

Formosa

Assam

Tenasserim

Tenasserim

S. Borneo

Assam

India

Siam

Andamans

Formosa

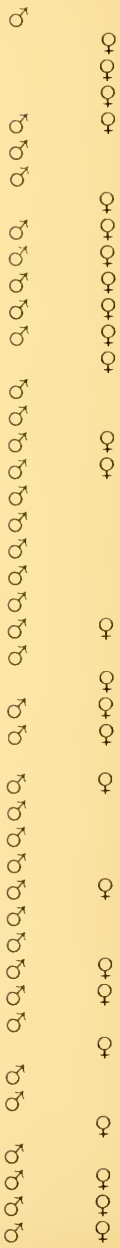




\section{Indo-Malayan (Continued)}

Lethe europa kumara

$$
\begin{aligned}
& \text { mahanava } \\
& \text { galaca } \\
& \text { mekara f. vajra } \\
& \text { " zuchara } \\
& \text { " crijnana } \\
& \text { " sumati } \\
& \text { " debata } \\
& \text { " gôpaka }
\end{aligned}
$$

Neope lacticolora

$$
\text { " pulaha didia }
$$

Neorina crishna archaica

" lowi pupillata

" neophyta

n " latipicta

Melanitis amabilis kajelana

$$
\begin{array}{cc}
\text { phaedima polishana } \\
\text { " } & \text { linga } \\
" & \text { patra } \\
\text { " } & \text { umkata } \\
" & \text { sumati } \\
\text { " } & \text { enganica } \\
\text { atrax erichsonia f. pseudaswa } & \text { " erichsonia } \mathrm{f} \text {. violetta } \\
\text { " bazilana } \\
\text { " lucillus } \\
\text { leda offaka }
\end{array}
$$

Erigano

Lombok

Palawan

Sikkim

Assam

Tonkin

N. Borneo

Sumatra

Malacca

Formosa

Tonkin

Tonkin

Assam

Padang

Palawan

W. Java

Batjan

Buru

S. Celebes

Sula

Ceylon

China (Fuchow)

Lombok

Formosa

Assam

W. Java

Tonkin

Sumatra

Tonkin

Formosa

Formosa

Tenasserim

Nias

Malacca

Sumatra

Buru

Dutch New Guinea

Formosa

N. Celebes

Omei Shan

Tze-Chuan

Nias

Engano

Mindoro

Mindoro

Bazilan

Mindanao

Waigeu 


\section{Indo-IMalayan (Continued)}

Melanitis leda destitans

$$
\begin{array}{ll}
\text { 1" } & \text { dominans } \\
\text { " } & \text { kiriwina } \\
\text { " } & \text { solomonensis } \\
\text { " } & \text { palliata } \\
\text { " } & \text { moluccarum } \\
\text { " levuna } & \\
\text { constantia f. depicta } \\
\text { " } \quad \text { obiana } \\
\text { " } \quad \text { dictatrix } \\
\text { " } \quad \text { batjana } \\
\text { " } \quad \text { minuscula } \\
\text { " } \quad \text { jobina } \\
\text { " } \quad \text { meforica } \\
\text { " } & \text { geluna } \\
\text { " } & \text { kapaura }
\end{array}
$$

zitenius zenon

$$
\begin{aligned}
& \text { f. depupillata } \\
& \text { f. decolorata } \\
& \text { semifasciata } \\
& \text { sumatranus } \\
& \text { auletes } \\
& \text { niasicus } \\
& \text { rufinus }
\end{aligned}
$$

belinda cruentata

boisduvali pompeja

palawanica

ernita

pyrrha orinoe

phaedima galkissa

ganapati

leda desperata

f. asuvina

Erites argentina ines

I'alaeonympha opalina macropthalma

Ragadia crisia siphonta

$$
\begin{aligned}
& \text { minerva } \\
& \text { pallida } \\
& \text { umbrata } \\
& \text { mindorana }
\end{aligned}
$$

Ypthima perfecta acragas

savara tonkiniana

conjuncta formosana

mistra zaboe

newara sarcaposa

motschulskyi ganus

baldus gallienus
Germ ". New Guinea

N. lreland

Trobriands

Solomons

Palau ls.

Amboina (male)

Batjan (female)

Fiji

Saparua

Obi

Buru

Batjan

Key

Jobi

Mafor

"Cerm n. New Guinea

Kapaur

Sumbawa

Sikkim

Sikkim

Sikkim

Sumatra

Tonkin

Nias

S. E. Borneo

Lombok

Bazilan

Palawan

Sangir

Batjan

Kulu

Tonkin

Tonkin

Wetter

S. Borneo

Formosa

Perak

Sumatra

Sumatra

Borneo

Mindoro

Formosa

Tonkin

Formosa

Formosa

Tenasserim

Tsingtau

Hainan 


\section{Indo-Malayan (Continued)}

Ypthima baldus scotia

ishigakina

f. zodina

horsfieldi morus

selimontius

aphnius saravus

1) caratonus

glabrius

bandinus

ninyas

aretas

stellera galaca

argus hypampeia

gangamela

asterope annamitica

$$
\text { n minorata }
$$

arctous papuana

fasciata torone

corticaria f. alada

pandocus moenius

certonus

calamus

maerius

andokides

chaborus

macrianus

anana

pandocus ancus

Harsiesis hygea jobina

$$
\begin{array}{ll}
\text { " } & \text { yolanthe } \\
\text { " noctula }
\end{array}
$$

Acropthalma artemis machares

$$
\text { " " lavina }
$$

Xois sesara vitiensis

Hypocysta isis

$$
\begin{aligned}
& \text { " salazar } \\
& \text { aroa serapis } \\
& \text { busilis } \\
& \text { haemonia fenestrella }
\end{aligned}
$$

Hong-Kong

Formosa

Formosa

Sumatra

Borneo

Lombok

Bali

Flores

Alor

N. Celebes

Saleyer

Palawan

Ussuri

E. Java

Annam

S. Celebes

"Germ n. New Guinea

Padang

Sumatra

Natuna

S. Borneo

Jolo

Nias

E. Java

Bazilan

N. Celebes

E. Celebes

S. Celebes

Jobi

Dutch New Guinea

"Germ" New Guinea

Bazilan

Sula

Fiji

"Germ" New Guinea

Dutch New Guinea

Dutch New Guinea

Misol

Dutch New Guinea 


\section{ELYMNIINAE}

Elymnias nigrescens tonkiniana

$$
\begin{aligned}
& \text { " " f. depicta } \\
& \text { formosana } \\
& \text { melilophila } \\
& \text { sumbawana } \\
& \text { sumbana } \\
& \text { orientalis } \mathrm{f} \text {. dohertyi } \\
& \text { timorensis } \\
& \text { beatrix } \\
& \text { beatrix } f \text {. decolorata } \\
& \text { beatrix } f \text {. ornamenta } \\
& \text { edela } \\
& \text { "f. pseudagina } \\
& \text { hecate } f \text {. virilis } \\
& \text { congruens phaios } \\
& \text { ") rufaela } \\
& \text { violetta } \mathrm{f} \text {. epixantha } \\
& \text { " }
\end{aligned}
$$$$
\text { hypermnestra meridionalis }
$$$$
\text { ") }
$$$$
\text { 1) }
$$$$
\text { " }
$$$$
\text { f. orphria }
$$$$
\text { tinctoria f. paraleuca }
$$$$
\text { baliensis }
$$

panthera teutra

$$
\begin{aligned}
& \text { f. defasciata } \\
& \text { aefredi } \\
& \text { " f. pantherina }
\end{aligned}
$$

lacrimosa

lacrima

agondas moranda

muscosa

aruana

milagondas

))

f. taenarides

sempyra

hagias

melanthes $f$. virginalis australiana

hewitsoni meliophila

hicetas hicetina

$$
\text { bonthainensis }
$$

butana

" vitellia f. basinus

$$
\text { " " suavinum }
$$

cybele obiana

$$
\text { adumbrata }
$$

ternatana

opaca
Tonkin

Tonkin

Formosa

Lombok

Sumbawa

Sumba

Eudé

Timor

Malay Pen

Sumatra

Perak

S. Borneo

Sarawak

N. Borneo

Mindanao

Bazilan

Siam

Siam

Annam

Annam

Tenasserim

Bali

Sumatra

N. Borneo

N. Borneo

N. Borneo

Bawean

?

Waigeu

Kapaur

Aru

Milne Bay

Milne Bay

Kumusi

Dutch New Guinea

Woodlark

Queensland

Saleyer

E. Celebes

S. Celebes

Butan

Saparua

?

Obi

Buru (male)

Amboina (male)

Ternate

Halmaheira 


\section{Elymniinae (Conlinued)}

Elymnias malelas f. subdecorata

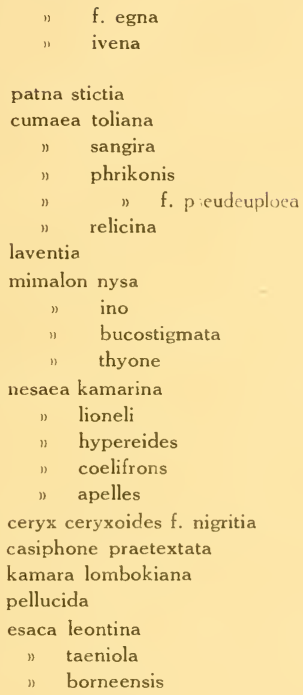

Assam (male Sikkim (female)

Sikkim

Tonkin (male)

Siam (female)

Tonkin

C. Celebes

N. Celebes

Sangir

Sula

Sula Besi

Waigeu

E. Celebes

C. Celebes

N. Celebes

N. Celebes

Batu

Malacca

N. Borneo

S. Borneo

Siam

W. Sumatra

Lombok

Lombok

N. Borneo

Nias

?

S. W. Borneo

\section{SATYRIDAE (African)}

Melanitis leda africana n zitenides
Pangani

Uganda 


\section{LIBYTHIINAE}

Libythea lepita matsumurae

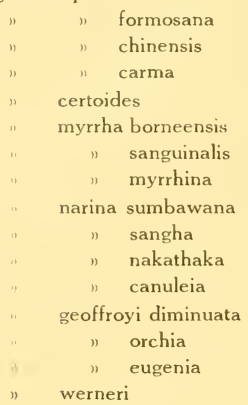

Japan

Formosa

Tze-Chuan

S. India

Japan

S. E. Borneo

Sikkim

Sumatra

Sumbawa

Sumba

Ceram

N. Celebes

Damer

Waigeu

New Guinea

Africa (Usambara)

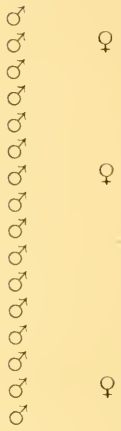

ERYCINIDAE

N. E. Sumatra

Borneo

N. Borneo

N. Borneo

N. E. Sumatra

S. Borneo

N. E. Sumatra

Okinawa

Siam

Tonkin

Annam

Bazilan

Tonkin

Mindoro

N. E. Sumatra

Tenasserim

N. Borneo

Nias

W. Java

Nias

Palawan

Rioun

N. E. Sumatra 


\section{Erycinidae (Continued)}

Abisara kausambi tera

savitri strix

" sciurus

neophron gratius

celebica bugiana

" juana

burni timaeus

" assus

11 etymander

haguinus certhae

$$
\text { n duras }
$$

" agias

thuisto ephorius

1) eutyches

n thericles

dora

Praetaxila statira maram

$$
\begin{gathered}
\text { " vedalla } \\
\text { " gudula } \\
\text { satraps bahadeva } \\
\text { segecia yaniya } \\
\text { " punctaria }
\end{gathered}
$$

Stiboges nymphidia mara

calycoides elodinia

Zemeros flegyas indicus

$$
\text { annamensis }
$$

phyliscus

sparsus

armazes

celebensis

sosiphanes

f. esla

hastius

emesoides zynias

$$
\text { " eso }
$$

Sospita fylla f. fyllaria

$$
\text { " " magdala }
$$

Dicallaneura decorata cigala

$$
\text { stagia }
$$

adulatrix

conos

kirschi didica ribbei arfakensis pulchra vasatha
N. Borneo

N. Borneo

S. Borneo

Tenasserim

N. Celebes

Mindanao

Tonkin

Canton

Formosa

Siam

N. E. Sumatra

Borneo

Siam

Borneo

Sumatra

Tonkin

Kapaur

"Germ" New Guinea

Waigeu

"Germ" New Guinea

lule I

Aru

N. Sumatra

W. Java

Tze-Chuan

Sikkim

Annam

Sumatra

Nias

Lombok

C. Celebes

S. Celebes

Annam

N. Borneo

N. Sumatra

S. Borneo

Sikkim

Tonkin

Yule 1 .

Dutch New Guinea

Waigeu

New Guinea

Dutch New Guinea

New Guinea

Kapaur 


\section{HESPERIIDAE}

Casyapa corvus corippus

$$
\text { aristippus }
$$$$
\text { rullus }
$$

L.ychnucus irvinus Ploetz (type of this)

Erionota hasdrubal

Paduka toradja

Tagiades japetus enganicus

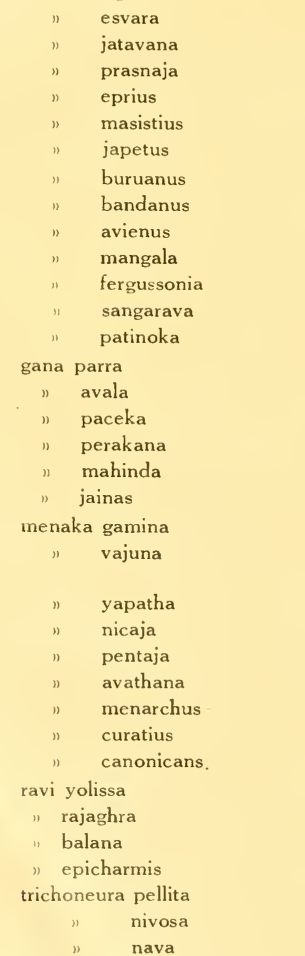

princeps bazilanus

paradonus

pteria dimidiata

Notocrypta feisthameli sanngutta

dharana
Fergusson

Buru

Milne Bay

E. Celebes

Batjan

E. Celebes

Engano

Lombok

W. Sumatra

S. Celebes

$\mathrm{Obi}$

Batjan

Halmaheira

Buru

Banda

Key

Babber

Fergusson

Tonkin

Siam

N. Borneo

W. Sumatra

Palawan

Malacca

N. Borneo

Nias

Tonkin

Karwar (male)

Malabar (female)

Nias

Bazilan

Buru

Sula

"Germ" New Guinea

ర大

0

o

o

$0^{x}$

o

$\sigma^{x}$

o

o

$0^{x}$

$0^{x}$

o

$0^{x}$

$0^{x}$

రิ

$0^{x}$

$0^{x}$

$0^{x}$

$\sigma^{x}$

$0^{x}$

o

$\pi$

"Germ" New Guinea

New Guinea

Nias

S. Borneo

N. Borneo

Siam

Tonkin

Nias

W. Java

Bazilan

N. Borneo

N. Borneo

Lombok

Sumbawa

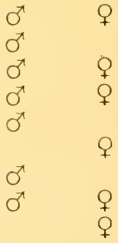

0
0

$\sigma^{\pi}$

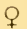




\section{Hesperiidae (Continued)}

Notocrypta feisthamelin samana

$$
\begin{aligned}
& \text { " satra } \\
& \text { waigensis mangala } \\
& \text { " ribbei } \\
& \text { alysos devadatta } \\
& \text { " sidha } \\
& \text { " asava } \\
& \text { " clavatta f. chunda } \\
& \text { " sukavata } \\
& \text { renaldi partita } \\
& \text { " fergussonia }
\end{aligned}
$$

Hasora badra godana

$$
\begin{aligned}
& \text { " inana } \\
& \text { anura avajra } \\
& \text { " teutra } \\
& \text { mixta yanuna } \\
& \text { " prabha } \\
& \text { cirta } \\
& \text { leucospila parnia } \\
& \text { " mastica } \\
& \text { discolor mastusia } \\
& \text { cridatta viliva } \\
& \text { doleschalli sitiva } \\
& \text { " viridicans } \\
& \text { malayana bhavana } \\
& \text { " avakra } \\
& \text { " padrua } \\
& \text { alexis vairacana } \\
& \text { " ganapata } \\
& \text { ribbei apara } \\
& \text { " dipama } \\
& \text { hurama vivahama }
\end{aligned}
$$

Ismene jaina formosana

$$
\begin{aligned}
& \text { " vasundhara } \\
& \text { ataphus athena } \\
& \text { harisa moncada }
\end{aligned}
$$

$$
\begin{array}{ll}
\text { " samara } \\
\text { n crinatha }
\end{array}
$$$$
\text { 11 asambha }
$$

gomata vajra

$$
\text { " calita }
$$

vasuntana rahita

oedippus aphrodite

nestor zonaras

amara pindapatra

Telicota nihana
Kapaur

Buru

New Guinea

Key

W. Sumatra

W. Java

Tonkin

Palawan

Sumbawa

New Guinea

Fergusson

Formosa

N. Borneo

Sumbawa

Nias

Nias

Palawan

Bazilan

N. Borneo

Bazilan

Queensland

Nias

Waigeu

Ceram

Assam

Sula

Palawan

Bazilan

Formosa

"Germ" New Guinea

Obi

Waigeu

Saparua (male)

Amboina (female)

Formosa

Assam

Siam

Malacca

W. Sumatra

W. Java

Tonkin

W. Java

W. Sumatra

Assam

N. Celebes

Wetter

Assam

Waigeu 


\section{Hespe:iidae (Continued)}

Telicota tamus yashtivana

terranea

dara anupya

" sapitana

" ahastina

" ganda

tropica dushta

sunias nikaja

gola trishna

dobboe maforica

bambusae formosana

$\begin{array}{ll}\text { " } & \text { rasana } \\ \text { " } & \text { upadhama } \\ \text { " } & \text { rahula } \\ \text { " } & \text { obiensis } \\ \text { " } & \text { subha }\end{array}$

paceka

dipavansa

ahamika

rudha

Cupitha purrea alara

$$
\text { n n. ogalana }
$$

Taractrocera ziclea sandvadha

$$
\begin{array}{ll}
\text { " } & \text { " ikramana } \\
\text { " } & \text { sudodana } \\
& \text { archias kisaga }
\end{array}
$$

(tenoptilum angulata mahabini

$$
\begin{array}{lcc}
\text { " } & \text { " } & \text { sumatrana } \\
\text { " } & \text { subangulata } \\
\text { " } & \text { pygela } & \text { ragupta }
\end{array}
$$

Caprona syrichthus helias

$$
\begin{array}{cl}
\text { " } & \multicolumn{1}{c}{\begin{array}{c}
\text { erosula helliger } \\
\text { alida mettasuta }
\end{array}} \\
\text { Parnara mehavagga } \\
\text { " } \quad \begin{array}{l}
\text { avastu } \\
\text { " }
\end{array} \\
\text { " } \quad \text { philippincta strativa mangolina } \\
\text { " } \quad \text { nirvana jetavana } \\
\text { " } \quad \text { yandova } \\
\text { " bipunctata sidata } \\
\text { " mathias niasica } \\
\text { " miltias miltiades } \\
\text { atropatene } \\
\text { icosia }
\end{array}
$$

Rhopalocampta benjamini formosana

Koruthaialos hanites namata

$$
\text { niasicus }
$$

Ceram

New Guinea

Formosa

Lombok

N. Borneo

Nias

Annam

N. Celebes

Nias

Mafor

Formosa

Palawan

Lombok

S. Celebes

Obi

Kapaur

"Germ" New Guinea

Waigeu

Waigeu

"Germ" New Guinea

C. Celebes

Andamans

Burma

S. Java

Sumba

Lombok

W. Java

N. Sumatra

Bazilan

W. Sumatra

Tonkin (male)

Indochina (female)

Indochina

Annam

S. Celebes

Formosa

Amboina

Sula

Bazilan

Formosa

Buru

Nias

New Guinea

Waigeu

"Cerm" New Guinea

Formosa

W. Sumalra

Nias
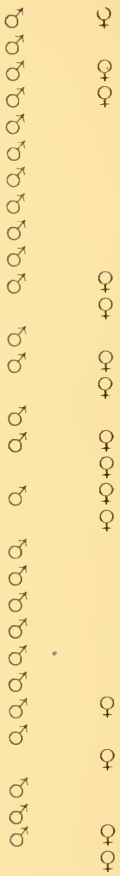

ox

$\sigma^{x}$

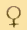

б

$0^{x}$

б

$\sigma^{x}$

o

0 


\section{Hesperiidae (Continued)}

Koruthaialos avidha

Annam

Ampittia formosana

Formosa

Ochus subvittatus intricatus

Tonkin

Pedestes parnaca

Isoteinon lamprospilos formosanus

Tonkin

Formosa

Kerana diocles sumata

Nias

S. Java

Bazilan

N. Borneo

Siam

N. Borneo

N. Borneo

Tonkin

Tonkin

Bazilan

N. Borneo

N. Borneo

Tonkin

Nias

W. Java

Bawean

Palawan

Bazilan

N. Borneo

S. Celebes

New Guinea

W. Sumatra

W. Java

Annam

Formosa

Java

Annam

Tonkin

Formosa

Jolo

Bazilan

Assam

Isma idyalis nipata

Matapa distanti niasica

Nias

N. Borneo

Nias

Plastingia niasana

Natuna

S. Celebes

C. Celebes

Sula

New Guinea

New Guinea

W. Sumatra

Charmion ficulnea nibana

Formosa

Sabara carina

1) caesina basina

Celaenorrhinus davidi ermasis 


\section{Hesperiidae (Continued)}

Celaenorrhinus leucocera philesar

$$
\begin{aligned}
& \text { aurivittata mahava } \\
& \text { asmara aditta } \\
& \text { consanguinea rata } \\
& \text { maculicornis piepersi } \\
& \text { "n vitruvius } \\
& \text { snelleni formosanus } \\
& \text { chamunda anoma }
\end{aligned}
$$

Coladenia dan sumatrana

$$
\begin{aligned}
& \text { " " dhyhana } \\
& \text { " lombokiana } \\
& \text { " celebica }
\end{aligned}
$$

Satarupa gopala tonkiana

$$
\begin{array}{ll}
\text { " sanbara indosinica } \\
\text { " celebica nivescens } \\
\text { (? genus) abstrusus } \\
\text { " formosana }
\end{array}
$$

Crossiura pencillatum kiyila
N. E. Sumatra

N. E. Sumatra

Siam

Formosa

W. Java

Siam

Formosa

S. Celebes

S. Celebes

W. Sumatra

Siam

Lombok

S. Celebes

Tonkin

Tonkin

S. Celebes

New Guinea

Formosa

Canton

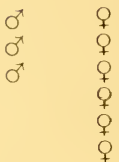

ర大

$0^{x}$

$\sigma^{x}$

o

$0^{x}$

o

$\sigma^{x}$

0

б

Q

Q

$0^{x}$

0

$0^{x}$ 


\section{LYCAENIDAE}

Thysonotis sophron

$$
\begin{gathered}
\text { " danis philocrates } \\
\text { " } \\
\text { " } \\
\text { " } \quad \text { phoibides } \\
\text { " } \\
\text { " proedrus reverdini }
\end{gathered}
$$

Eupsychellus euchylas ariel

$$
\begin{array}{cc}
\text { " } & \text { phasis } \\
\text { " hyphasis } \\
\text { alaenas sarsina }
\end{array}
$$

Niphanda fusca tituria

$$
\text { " shiguma }
$$

Lampides amphissa leiothrix

$\begin{array}{lll}" & n & \text { viosa } \\ n & n & \text { courvoisieri } \\ n & n & \text { hellada } \\ " & n & \text { mardada } \\ n & n & \text { zelea } \\ n & n & \text { megdara }\end{array}$

philatus arins

$$
\begin{array}{ll}
\text { " } & \text { aroaseins } \\
\text { " } & \text { andrus } \\
\text { " } & \text { armatens } \\
\text {, } & \text { nemithus } \\
\hline & \text { nemea sanaya }
\end{array}
$$

kondulana paliassa

$$
\begin{aligned}
& \text { "l f. ateina } \\
& \text { crocula } \\
& \text { thalaba } \\
& \text { yapa } \\
& \text { phaliga } \\
& \text { baweana } \\
& \text { comeda }
\end{aligned}
$$$$
\text { sammda }
$$

kankena silvagia

aditja

abdul hamid

1) daomides Rober

alsietius

callistus cleitus

$$
\text { " neaothrus }
$$$$
\text { " amastris }
$$

elpis dromicus

Lampides elpis rajatus

$$
\begin{aligned}
& \text { " meilidrinus } \\
& \text { " eurysares f. ozea } \\
& \text { (1) thanetus } \\
& \text { ") eurysthenes }
\end{aligned}
$$

Buru

Obi

Mafor

Brit. New Guinea

Queensland

Brit. New Guinea

Key

Roon

New Guinea

Aru

China

Japan

Engano

Lombok

Nias

Sula

Key

S. Celebes

$\mathrm{Obi}$

N. E. Sumatra

Nias

Bazilan

?

Waigeu

Obi

Sikkim

Sikkim

Andamans

N. Borneo

?

Bazilan

Bawean

Tanah Djampea

Obi

Simalur

N. Borneo

Nias

W. Java

Bazilan

Bazilan

Palawan

Mindanao

Formosa

Tonkin

Ceylon

Sikkim

Nias

Sumbawa 


\section{Lycaenidae (Continued)}

Lampides elpis sumbanus

n artemitus

$$
\begin{aligned}
& \text { ageladas } \\
& \text { alearto } \\
& \text { aloannus } \\
& \text { eurysares } \\
& \text { argentiferus } \\
& \text { demetrias } \\
& \text { cytinus }
\end{aligned}
$$

celeno lygdamus

$$
\begin{array}{ll}
\text { " } & \text { tissana } \\
\text { " } & \text { arama } \\
\text { " } & \text { ruvana } \\
\text { " } & \text { sandya } \\
\text { " } & \text { sandana } \\
\text { " } & \text { f. parazebea } \\
\text { " } & \text { f. parazebea } \\
\text { " } & \text { f. elpimides }
\end{array}
$$

rama f. zebrina

vaneeckei

cleodus trichonis

$$
\begin{array}{ll}
\text { "1 } & \text { semperi } \\
\text { " } & \text { mamias } \\
\text { " } & \text { potic'alon } \\
\text { " } & \text { derrhis } \\
\text { " } & \text { lydannus }
\end{array}
$$

suidas zelinus

$$
\text { " eordaa }
$$$$
\text { aratus adana }
$$

$$
\text { nansiphanes }
$$$$
\text { minthe }
$$

pseudaratus aetherialis vininga

$$
\text { ") durus }
$$

Nacaduba berenice zygida

$$
\text { aphya }
$$$$
\text { eurytius }
$$$$
\text { ceylonica }
$$$$
\text { aphana }
$$$$
\text { icana }
$$$$
\text { eliana }
$$$$
\text { zyrthis }
$$$$
\text { carnarina }
$$

angusta thespia

pactolus continentalis
Sumba

Palawan (male)

N. Borneo (female)

Sumatra

Buru

Saleyer

Assam

N. Celebes

N. Celebes

Brit. New Guinea

Formosa

Ceylan

Nias

Lombok

Halmaheira

Key

S. Java (male)

W. Java (female)

Assam

Nias

Nias

Palawan

Mindoro

Mindanao

Bazilan

Formosa

N. Celebes

N. E. Sumatra

Palawan

N. Borneo

Natuna

Palawan

Sula

Obi

Halmaheira

Dutch New Guinea

Dampier

Palawan

Siam

India

Ceylon

Nias

N. E. Sumatra

N. Celebes

Sumba

Obi

Banguey

Sikkim 


\section{Lycaenidae (Continued)}

Nacaduba pactolus andamanica

$$
\begin{aligned}
& \text { " odon } \\
& \text { pavana sidama } \\
& \text { n asiaga } \\
& \text { n sanaya } \\
& \text { 1) metallica } \\
& \text { " " f. visma }
\end{aligned}
$$

pernsia euplea

$$
\text { " therasia }
$$$$
\text { " parachutea }
$$$$
\text { " astapa }
$$$$
\text { n) laronia }
$$$$
\text { atrata neon }
$$$$
\text { " gythion }
$$$$
\text { " jedja }
$$$$
\text { " asakusa }
$$

palmyra cythora

$$
\begin{aligned}
& \text { " vaneeckei } \\
& \text { hyperesia } \\
& \text { aluta lessina } \\
& \text { " philiata } \\
& \text { " alutina } \\
& \text { ardates meraha } \\
& \text { nora kupu Kheil. } \\
& \text { " semperi } \\
& \text { " formosana }
\end{aligned}
$$

Jamides plato f. nava

bochus nabonassar

$$
\text { " phameges }
$$

bochus hordomius

$$
\begin{array}{ll}
\text { n } & \text { polassar } \\
\text { 3) } & \text { toxinus } \\
\text { " } & \text { tiglath } \\
\text { " } & \text { herodicus } \\
\text { " } & \text { phaidon }
\end{array}
$$

Lycaenopsis cossaea hegesias catreus hermeras quadriplaga nearcha nedda labranda " gradenija vesontia albocoeruleus vianus

$$
\text { n aristeus }
$$

Rapala alcetas ingana kessuma throana selira roana
Andamans

N. E. Sumatra

S. India

N. Borneo

Nias

N. Celebes

S. Celetes

Sikkim

Formosa

Nias

Bali

Lombok

N. Sumatra

Assam

Nias

Formosa

Batjan (male)

Obi (female)

Cent. New Guinea

$\mathrm{Obi}$

Nias

Bazilan

N. Celebes

Lombok (male)

Sumbawa (female)

Nias

Bazilan

Formosa

Sikkim

Borneo

Lombok

Wetter

Obi

Halmaheira

Sula

Luzon

S. Celebes

Nias

E. Java

N. E. Sumatra

Amboina

E. Celebes

Mindoro

N. E. Sumatra

S. Celebes

N. Borneo

Borneo

India (Bashahr)

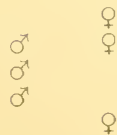

ర大

(n)

(n)

(n)




\section{Lycaenidae (Continued)}

Rapala suffusa catilus

" epijarbas manartius

$$
\begin{array}{lll}
n & \text { " } & \text { aureus } \\
" & \text { "1 } & \text { trenzius } \\
" & \text { " } & \text { corrolanus } \\
" & \text { " } & \text { mesarchus } \\
\text { " } & \text { " } & \text { megakles } \\
\text { " } & \text { staudingeri sumatrensis }
\end{array}
$$

Lycaenopsis puspa gisca

$$
\begin{array}{cl}
\text { puspa } & \multicolumn{1}{c}{\text { myla }} \\
\text { n } & \text { hermagoras } \\
\text { " } & \text { telus } \\
\text { " } & \text { turdeta } \\
\text { " } & \text { mygdonia } \\
\text { " } & \text { sania } \\
\text { " } & \text { darnis } \\
\text { " contilia } \\
\text { " valata } \\
\text { " hermione } \\
\text { " roeberi } \\
\text { " kühni Rober. } \\
\text { " najara } \\
\text { ottonis } \\
\text { " bazilana } \\
\text { " sabis } \\
\text { " georgis } \\
\text { akasa mavisa } \\
\text { " catullus } \\
\text { " calous }
\end{array}
$$

Spindasis syama rubicunda

$$
\text { lohita seliga }
$$

Tajuria jangala ravata $f$. phaedra

$\begin{array}{lll} & \text { " } & \text { centa } \\ " & \text { " } & \text { huberta } \\ \text { " } & \text { mundra } \\ & \text { " tinena }\end{array}$

Hypochrysops prthias werneri

$$
\begin{gathered}
\text { polycletus menyllus } \\
\text {, oinens } \\
\text { " kylatlus } \\
\text { kaystius }
\end{gathered}
$$

eucletus menandrus

$$
\text { n eratosthenes }
$$

polycletus linos
Nias

Tonkin (male)

Assam (female)

Sikkim

Nias

Palawan

Lombok

S. Celebes

W. Sumatra

Sikkim

Sikkim

Formosa

Hainan

Ardamans

N. Borneo

N. E. Sumatra

Lombok (male and female)

Java (male and female)

Key

Sumbawa

Flores

Bawean

Goram

S. Celebes

S. Celebes

Palawan

Bazilan

Mindoro

Mindanao

India

Sumatra

Sumbawa

N. Borneo

Tenasserim

Assam

Nias

N. Borneo

Hong-Kong

S. Celebes

"Germ" New Guinea

Halmaheira

Dutch New Guinea

Malor

"Germ" New Guinea

Waigeu

Brit. New Guinea (male)

Dutch New Guinea (female)

Roon
Q

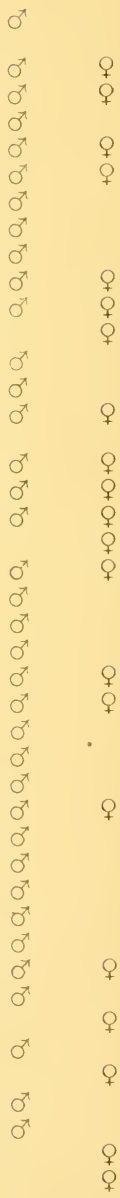

$0^{x}$
0 


\section{Lycaenidae (Continued)}

Hypochrysops vosoia

$\begin{array}{ll}\text { "n } & \text { aurigena } \\ \text { doleschalli modocans } & \text { pretiosus aristobul. }\end{array}$

Lophyrus japonica f. amethystina

Heliophorus epicles indicus

\begin{tabular}{lll}
$n$ & " & \multicolumn{1}{c}{ f. raufonalates } \\
$n$ & " & f. latilimbata \\
$n$ & $n$ & sumatrensis \\
$n$ & $n$ & matsumurae
\end{tabular}

lraota rohana $f$. aenus

Thaduka ameria formosae

$$
\text { " "zistra }
$$

Amblypodia narada fara

$$
\begin{aligned}
& \text { " } \text { saloia } \\
& \text { " } \text { orla } \\
& \text { " } \text { dina } \\
& \text { anetta } \text { eberalda } \\
& \text { " } \text { elga } \\
& \text { " fabiana }
\end{aligned}
$$

Surrenda vivarra samina

$$
\begin{array}{lcc}
\text { " } & \text { amisena agrostis } \\
n & n & \text { deverra } \\
\text { " } & \text { myrleia } & \text { myercetorum neritos }
\end{array}
$$

Arhopala hercules stymphelus

$$
\begin{aligned}
& \text { " sopatrus } \\
& \text { " } \quad \text { pontinus } \\
& \text { tyrannus afranins } \\
& \text { " sophilus } \\
& \text { centaurus çervidens } \\
& \text { " contenitus } \\
& \text { sphetlyo } \\
& \text { hellada } \\
& \text { " ozana } \\
& \text { adoria gorgias } \\
& \text { phaenops termerion } \\
& \text { micale alkisthenes } \\
& \text { meander amicans } \\
& \text { " maditus } \\
& \text { "gazclea } \\
& \text { hylander dylas } \\
& \text { " parnes } \\
& \text { aexone herana } \\
& \text { " natanda } \\
& \text { nobilis ajusa }
\end{aligned}
$$

Waigeu

"Germ" New Guinea

Queensland

"Germ" New Guinea

Japan

Sikkim

Sikkim

Sikkim

Padang

Formosa

S. Java

Formosa

Siam

Sumatra

N. Borneo

S. Java

Sikkim

Dutch New Guinea

Obi

Waigeu

N. Celebes

Nias

Borneo

N. Borneo

S. Annam (male)

Tonkin (female)

Batjan

Milne Bay

Misol

Jobi

Brit. New Guinea

Obi

Borneo

Batu

Nias

Nias

N. E. Sumatra

Java

Bazilan

"Germ" New Guinea

Solomons

Australia

New Britain

Obi

New Guinea

"Germ" New Guinea

Kiriwina

Galela Hannah 


\section{Lycaenidea (Continued)}

Arhopala nobilis nobilior amytis selymbria " androtion n leptines

vidona

anthelus majestatis n sotades

admete admetina

$$
\text { " } n \text { f. hellava }
$$

mindanaensis zilensis

asoka baya

diardi amaha

n zilana

fulgida zohar

amantes onetor

$$
\begin{array}{ll}
\text { nerelius } \\
\text { n } & \text { aphobus } \\
\text { n } & \text { pylagore }
\end{array}
$$

eupolis philtron cannlia sosias fulla prasia acron acronides thamyras potidaea

$$
\begin{array}{ll}
\text { " calaureia } \\
\text { "zeleae }
\end{array}
$$

thamyras tenthaone paramuta ? munijaga bazalus nebonius saoharga apidanus cerossus

$$
\begin{array}{ll}
\text { " } & \text { xisuthrus } \\
\text { " } & \text { heriya } \\
\text { himna } \\
\text { " } & \text { arahat } \\
\text { " cames }
\end{array}
$$

abseus oghatimna

$$
\text { nava }
$$

Cyaniris celestina f. Kobei Tutt.

argiolus cilion herophilus crimissa

limbatus hermesianase

$$
\begin{aligned}
& \text { gadara } \\
& \text { hellonia } \\
& \text { himilcon } \\
& \text { hegesander } \\
& \text { pellans }
\end{aligned}
$$

Obi

Waigeu

Yule 1

Key

Kirwina

Nias

Philippines

Obi

$\mathrm{Obi}$

Bazilan

Hong Kong

N. Borneo

Bazilan

N. Borneo

Sumbawa (male)

Sumba (female)

Kalao

S. Java

Sumatra

Yule I

Obi

Amboina

Obi

Obi

Misoli

Kirwina (male)

Woodlark (male)

Dutch New Guinea

Canton

N. E. Sumatra

S. Annam

Borneo

Nias

Bazilan

Mindanao

Bawean

Lombok

Bazilan

N. Borneo

Japan

Annam

Annam

Formosa

Mindanao

Obi

S. Celebes

Formosa

Tonkin

Lombok 


\section{Lycaenidae (Continued)}

Cyaniris limbatus placidina

$$
\begin{array}{lcl}
\text { " } & \text { " epicharna } \\
\text { " } & \text { idamis } \\
\text { " } & \text { cardia hermonthis } \\
\text { " } & \text { " hainana } \\
\text { " } & \text { " lombokensis } \\
\text { " } & \text { " paracatina } \\
\text { " } & \text { " masinassa } \\
\text { " } & \text { strophis cynanae } \\
\text { " } & \text { " euphon } \\
\text { " } & \text { musina pelides } \\
\text { " } & \text { camenae elothales } \\
\text { " } & \multicolumn{2}{c}{\text { valeria }} \\
\text { " } & \text { " jugurtha }
\end{array}
$$

Lycaenesthes emolus goberus

$$
\text { " " " ? soltattus Rober. }
$$

Luthrodes (Talicada) cleotas gades

$$
\text { " n " kaiphar }
$$$$
\text { " " boopis }
$$

Megisba malaya volulilis

$$
\begin{array}{lll}
\text { " } & \text { " } & \text { infumata } \\
\text { " } & \multicolumn{3}{c}{\text { rosanna }} \\
\text { " } & \text { zalmora colutha } \\
\text { " } & \text { " } & \text { indigeta } \\
\text { " } & \text { " } & \text { tituria } \\
\text { ") } & \text { " heria }
\end{array}
$$

Castalius ananda vilya

$$
\text { ethion ethionoides }
$$

$$
\text { " gadamas }
$$$$
\text { " sangarius }
$$$$
\text { 1) icenus }
$$

roxus manovus

$$
\text { n continentalis }
$$

Castalius rexus satrannus

$$
\begin{aligned}
& \text { " pothus } \\
& \text { " odon f. nisana } \\
& \text { elna noliteria } \\
& \begin{array}{lll}
\text { " } & \text { f. elina } \\
\text { " hilina } & \\
\text { " eloira . }
\end{array}
\end{aligned}
$$

Gerydus horsfieldi hermaguus

$$
\begin{aligned}
& \text { infumata } \\
& \text { intricata } \\
& \text { satelliticus } \\
& \text { apries } \\
& \text { reverdini }
\end{aligned}
$$

posidion attacinus

$$
\text { n eurytanus }
$$

N. E. Sumatra

Flores

Sumatra

Formosa

Hainan

Lomb̌ok

Borneo

Flores

Lombock

S. Celebes

Dran (Annam)

N. E. Sumatra

S. Celebes

Flores

N. Borneo

New Guinea

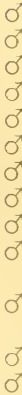

Dutch New Guinea

Shortland 1 .

Milne Bay

N. Celebes

Formosa

S. Celebes

Mindoro

Palawan

Bawean

Tanah Djampea

Kiriwina

N. E. Sumatra

W. Sumatra

Lombock

Bawean

N. Borneo

Borneo

Tonkin

Luzon

Sumatra

Sumbawa

Sikkim

Sikkim

Nias

Sumatra

W. Sumatra

Sumatra

N. E. Sumatra

Engano

N. Borneo

W. Borneo

Burma

Borneo 


\section{Lycaenidae (Continued)}

Gerydus posidion molionides unicolor enganicus

$$
\begin{array}{ll}
\text { " cajanus } \\
\text { " damodar }
\end{array}
$$

leogoron

aphocha anatheus

$$
\text { n zitima }
$$

symethus acampsis

$$
\begin{array}{ll}
\text { " } & \text { deiopeithes } \\
\text { " } & \text { philopator } \\
\text { " megaris } \\
\text { " vespasianus }
\end{array}
$$

melanion bazilanus

$$
\text { " vitellianus }
$$

archelochus

eulus

leos tellus

$$
\begin{array}{ll}
\text { ") } & \text { ageles } \\
\text { " } & \text { sarus } \\
\text { " } & \text { cataleucus } \\
\text { " } & \text { mangolicus } \\
\text { " } & \text { gardineri } \\
\text { " amphiacus } \\
\text { " meranus } \\
\text { " virtus } \\
\text { " } \quad \text { f. pentheus }
\end{array}
$$

" acrisius

zinckeni valens

boisduvali milvius

$$
\begin{array}{cc}
n & \text { heragis } \\
n & \text { heraon } \\
n & \text { lombokianus } \\
n & \text { hyllus } \\
n & \text { avitus } \\
n & \text { epidurus } \\
\text { " } & \text { diatraphes } \\
\text { " } & \text { gethusus } \\
\text { courvoisieri phradimon } & \\
\text { learchus carinas } \\
\text { biggsi oichalea } \\
\text { " f. denticulata } \\
\text { " cellarius } \\
\text { " eustatius } \\
\text { " niasicus } \\
\text { " nymphes } \\
\text { " sebethus }
\end{array}
$$

Bali

Engano

Lombock

S. Celebes

N. E. Sumatra

Bali

N. Celebes

N. E. Sumatra

Rioun

?

Lombok

Nias

Bazilan

?

Tonkin

Sumbawa

?

E. Celebes

E. Celebes

Saleyer

Sula

Amboina

Bangkei

Ceram

Batjan

Batjan (male)

Halmaheira (female)

New Guinea

N. E. Sumatra

Sikkim

Singapore

Borneo

Lombok

N. E. Sumatra

Key

Palawan

E. Celebes

Dran (Annam)

Tonkin

N. Borneo (male)

W. Borneo (female)

W. Borneo

Java

N. E. Sumatra

N. Borneo

N. Borneo

Nias

Sumatra

W. Borneo

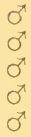

우

우

운

q

ㅇ

0
0
0
0

q

P

q

ㅇ 


\section{Lycaenidae (Continued)}

Gerydus biggsi aphtonius

fabius arrius n pamisus

martinus

strigatus Moult. " euphalion ndosithous

portunus maitus

taras sarrastes

") battakanus

maximus $f$. divisa

Logania subviolascens cilarus kallikrates

fallax ancius

1) dotion

nivalis magaris

n lenaia

marmorata stinosa

") hilaeira

domissa

massalia nada

n) sora

Orthomiella pontis revorea

Curetis thetis menestratus

" isabellae

insularis hera

ge

acuta japonica

") tsushimana

formosana

bulis fortunatus

Biduanda minara

lysias euthydemus

aurea cuprea

anasuga irimina

skopula sunkilia

tharis latipictus

thealoides extensa

Cheritra acte $f$. idina

Marmessus moorei nola

Zeltus etolus maximinianus

$$
\begin{aligned}
& \text { gratitianus } \\
& \text { pompadius } \\
& \text { censorinus }
\end{aligned}
$$

Hypolycaena erylus himavantus
Tenasserim

Sumatra

S. E. Borneo

Buton

N. Borneo

N. E. Sumatra

Java

N. E. Sumatra

N. Borneo

N. E. Sumatra

S. Celebes

N. Borneo

Mindanao

Mlindanao

Bazilan

N. E. Sumatra

Nias

Borneo

N. E. Sumatra

S. Celebes

N. E. Sumatra

N. Borneo

E. Burma

Dutch New Guinea

Bazilan

Nias

Nias

Japan

Tsushima

Formosa

Tonkin

S. Borneo

Cochinchina

N. E. Sumatra

Nias

N. E. Sumatra

Nias

Engano

Sikkim

N. Borneo (male)

Sumatra (female)

W. Sumatra

Nias

W. Java

Sumbawa

Tonkin (male)

Annam (female)

N. Celebes

Lombok 


\section{Lycaenidae:" (Continued)}

Hypolycaena pupuenus siphax

\begin{tabular}{|c|c|c|}
\hline " & $\Rightarrow \quad t$ & teatus \\
\hline ") & ") & pigres \\
\hline$"$ & $n$ & thyrius \\
\hline$n$ & $n \quad \mathrm{f}$ & figulus \\
\hline 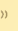 & phorbas & $\mathrm{s}$ tullus \\
\hline ") & $”$ & silo \\
\hline$n$ & " & infumata \\
\hline 1) & sipylus & numa \\
\hline$n$ & $"$ & rhodanus \\
\hline n & " c & capella \\
\hline$n$ & nedimon & nd anaximander \\
\hline$"$ & mergina & a sobanas \\
\hline
\end{tabular}

Loxura atymnus continentalis

\begin{tabular}{|c|c|c|}
\hline "1) & $"$ & f. inahara \\
\hline$n$ & ") & fiuronius \\
\hline$"$ & $"$ & leminins \\
\hline$"$ & 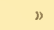 & matienus \\
\hline " & $n$ & anglerinus \\
\hline$n$ & $n$ & calintus \\
\hline " & $"$ & cassiopeia amatia \\
\hline$n$ & $n$ & fuscicaudata \\
\hline 》) & $n$ & diania \\
\hline
\end{tabular}

Chilaria vanavasa

"tora Kheil (? type verus)
" ister yasa
" lucida damara

Horaga onyx arta

$$
\begin{array}{lll}
" & \text { " privigna } \\
n & " & \text { paurrus } \\
n & \text { " chalcedonya } \\
" & \text { " saridonyx } \\
\text { " affinis artomtes } \\
\text { " cellula anara }
\end{array}
$$

Rathinda amor himeros

Catapoecilma major anais

$$
\text { elegans niasana }
$$

Lycaena hylas castilla

$$
\begin{aligned}
& \text { " alcon hypseus } \\
& \text { " arion delphinatus } \\
& \text { " } \\
& " \text { " laranda } \\
& " \text { vesubia } \\
& \text { " } \quad \text { plistion } \\
& \text { " euphenus thersandrus } \\
& \text { " icarus labynetus }
\end{aligned}
$$

Thecla ilicis incruentus
Nias

$\mathrm{Obi}$

N. Borneo

Halmaheira (male)

Batjan (female)

Waigeu

Solomons

"Germ" New Guinea

Vulcan 1.

Flores

S. Celebes

Lombock

IV. Sumatra

Borneo

Siam

Sikkim

N. Borneo

N. E. Sumatra

Lombock

Kișser

S. Celebes

N. Borneo

Nias

Mindanao

N. E. Sumatra

Formosa

Nias

Nias

Palawan

Assam

Lombok

Bazilan

Borneo

N. E. Sumatra

Nias

E. Java

Ceylon

Assam

Nias

Castile

Berchtesgaden

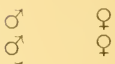

ช'

0

ठౌ

0

రె

o

8

$\sigma^{x}$

ర

o

o

ণ

o

o

dx

o

0

ot

o

o

o

q

q

రా

$0^{x}$

o

$q$

б

0

$\sigma^{\pi}$

$\sigma^{x}$

La Grave

Klausen

Alp. Mmes

Sajam

Juldus

Eclepens Switz.)

Palestine

Saratow 


\section{FRUHSTORFER COLLECTION}

SECOND LIST OF TYPES 



\section{PAPILIONIDAE}

Troides helena mamius andromache marapokensis amphrysus niasicus ikarus haliphron antilochus poseidon $f$. aurago
")
f. boreas
f. lavata

amphrysus euthydemus staudingeri Rôb.

Papilio priapus dilutus memnon ab. seriatus payeni brunei eurypylus daton sarpedon rufocellularis arjuna $f$. gedeensis ulysses oxyartes n kallinikos "f. roxana 1) ? tenocles ascalaphus $f$. nubiger caunus typhrestus hewitsoni f. persides echidna echidnides liris damarius

$$
\begin{aligned}
& \text { "geatus z } \\
& \text { " pseudoliris } \\
& \text { " senescens Rôb. } \\
& \text { velificatus }
\end{aligned}
$$$$
\text { clytia janus }
$$$$
\text { inopinatus inamis }
$$$$
\text { peranthus kinesias }
$$$$
\text { " phoebus }
$$$$
\text { „ fannrus }
$$$$
\text { 1) transiens }
$$

deiphobus aristartus jordani

pericles olympiodorus doson lucus " ampyx polyctor significans krishna charlesi thule $f$. viridicans " alfredi palinurus nymphodorus autosayus veiovis samanganus
Bali

N. Borneo

Nias

Selaru

Alor

Waigeu

Dutch N. Guinea

Waigeu

W. Sumatra

Serang

E. Java

W. Java

N. Borneo

W. Java

Bawean

Java

Aru

N. Ireland

N. Guinea

Milne Bay

N. Celebes

S. Borneo

N. Borneo

Moah

Dammer

Savu

Savu

Kisser

Roma

Siam

Babber

Dammer

Flores

Alor

Sumbawa

Lombok

Dutch N. Guinea

Celebes

Babber

Tanah Djanpea

Lombok

Tenasserim

Sia-lou

Waigeu

Waigeu

Bazilan

Japan

S. Celebes 


\section{Papilionidae (Continued)}

Papilio veiovis aristillis

$$
\begin{aligned}
& \text { alcinous mansonensis } \\
& \text { mahadeva phanrangensis } \\
& \text { canopus babberensis } \\
& \text { " kallon } \\
& \text { hipponus bazilanus } \\
& \text { hipponous leptopsephus } \\
& \text { payeni langsonensis } \\
& \text { gygas aribbas } \\
& \text { canopus kratidas }
\end{aligned}
$$

E. Celebes

Tongkin

S. Annam

Babber

Roma

Bazilan

Assam

Tongkin

Upp. Burma

Dammer

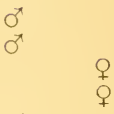

ర

$0^{x}$

c)

$0^{x}$

\section{S. AMERICA}

Papilio glaucolaus phenelaus

Honduras

" protesilaus expurgator

Espirito Santo

$0^{x}$

$0^{x}$ 


\section{PARNASSIUS}

F. apollo pedemontanus

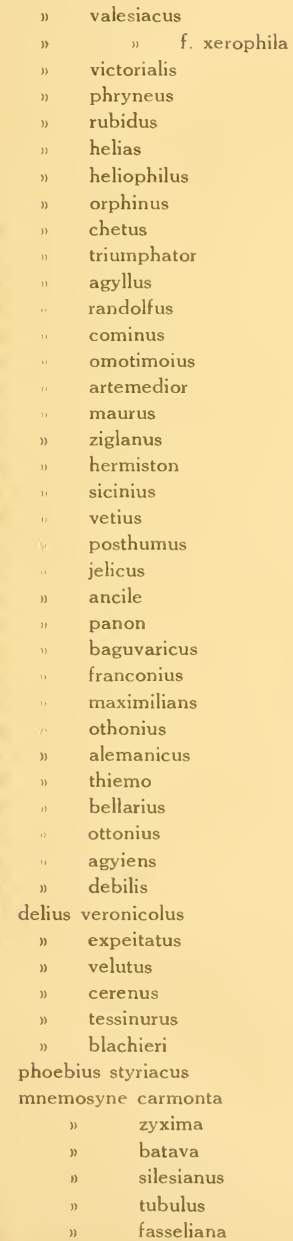

Courmajeur

ơ Simplon, ơ Wallis

Moutiers

Trient

Solden Oetz

Klausen

Flums

Val Blenio

Bavaria

Upp. Austria

Novaggio

Bunsio

Weissenbach

Carpathians

Bulgaria

Carpathians

Transylvania

Bulgaria

Schipka

Teschen

Walchen

Saalethal

Serbia

Bayern

"

")

Bavaria

o Kaisergebirge. $q$ Ammergau Grossen Kaiser

Fussen

Neu-ulm

Raabenstein

Kustenland

Andraz.

ऽ Thoreno, \& Haut Garon

Zermatt

Kummersee

Venediger

Alp du Piemont

Tessin

Veglia

Reichenstein

$\sigma^{\top}$ Vorarlberg $Q$ Krain

Wachau

Passau

Schlesien

Schoberstein

Strobnitz 


\section{Parnassius (Continued)}

$\begin{array}{cl}\text { mnemosyne leonhardiana } \\ \text { " } & \text { parmenides } \\ \text { " } & \text { thesoleucus } \\ \text { " } & \text { symphorus } \\ \text { " } & \text { mixtus } \\ \text { " } & \text { temora } \\ \text { " } & \text { tergestus } \\ \text { " } & \text { ariovis } \\ \text { " } & \text { f. subochracea } \\ \text { " } & \text { thaleia } \\ \text { ") } & \text { dinianus }\end{array}$

Wetti

Alp. Mar.

N. Ungarn

Val Maggia

Macugnaga

Lotsehenthal

Thalalpsee

Erstfeld

Ortler

ot Urach, $Q$ ?

Erstfeld

Tirol

Digne

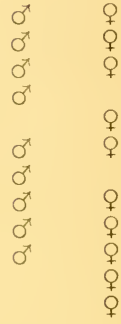

$\sigma^{x}$ 


\section{PIERIDAE}

Delias belladonna yedanula

$$
\begin{gathered}
\text { crithoe bromo } \\
\text { " dillia } \\
\text { " f. fastosa } \\
\text { " f. funesta }
\end{gathered}
$$

altivaga

nakula depicta

belisama auratilis

nakula f. atisha

$$
\text { f. yogini }
$$

henningia hemera pandemia saturnia aglaia goda

$$
\text { " grisea }
$$$$
\text { triglites }
$$$$
\text { cyrania }
$$$$
\text { tonkiniana }
$$

beata curasena

aruna arovana

$$
\text { " irma }
$$

callistrate scribonia

$$
\text { ") felsina }
$$

gabia mavroneira

georgina battana

$$
\text { " " f. aurigena }
$$

agostina infumata

$$
\text { " orita }
$$

singapura indistincta

blanca nausicca.

themis sotiera

dorimene avenda

odyssia

duris aleria

candida tuthrania

$$
\text { " antissa }
$$

mysis tenes

$$
\begin{array}{ll}
\text { " onca } \\
\text { " magica } \\
\text { " adelphae } \\
\text { " oisyme } \\
\text { " nemea } \\
\text { " alorensis }
\end{array}
$$

argenthona balli Hulst.

argenthona - f. seminigra hyparete peirene
Tze-Chuan

Omei Shan

E. Java

W. Sumatra

or S. Java, \& W. Java

W. Java

W. Java

E. Java

E. Java

E. Java

E. Java

?

N. Borneo

N. E. Sumatra

Nias

N. E. Sumatra

Hainan

Tonkin

$\sigma^{x}+$ Indo-China, $\sigma^{x}$ Tenasserim

Formosa

Rossel Is.

Milne Bay

New Guinea

Bongu

Kirivina

Kajumera

Aru

S. Celebes

S. Celebes

Tenasserim

Indo-China

S. Borneo

N. Borneo

Luzon

Ceram

Waigeu

Ceram

Obi

Halmaheira

"Germ. ", N. Guinea

Milne Bay

Rossel ls.

Yule Is.

Waigeu

Merauke

Alor

Merauke

Australia

Formosa 


\section{Pieridae (Continued)}

Delias hyparete peirene f. maenaa

bagoe nausana

timorensis gardeneri

poecilia edela

belladonna perspicua

agostina annamitica

hyparete ciris

") diva

" jataka

oraia vasumitra

alluviorum

descombsei $q$ f. leucogaea " eranthos

descombsei $f$. auriga leucacantha

Huphina euryxanthe caroli

$$
\begin{aligned}
& \text { " temena lenitas } \\
& \text { " } \quad \text { hylia calliparga } \\
& \text { " corva f. corvina }
\end{aligned}
$$

\begin{tabular}{|c|c|c|}
\hline ) & ” & f. \\
\hline & " & f. \\
\hline$n$ & philonome & $\mathrm{f}$. nivescen \\
\hline ") & " & f. plagiata \\
\hline ") & $n$ & f. herennia \\
\hline n & n & f. lactaria \\
\hline
\end{tabular}

Ixias reinwardti lombokiana

$$
\begin{aligned}
& \text { " " kangeana } \\
& \text { " " } \quad \text { f. noctula } \\
& \text { "baliensis } \\
& \text { "venila } Q \text { f. (white) }
\end{aligned}
$$

Prioneris autothysbe orientalis

Appias leis $\mathrm{f}$. $q$ obscurior

$$
\begin{aligned}
& \text { " " } \quad \text { f. atania } \\
& \text { " } \quad \mathrm{f} \text {. emblema } \\
& \text { " pandrone f. eburnea } \\
& \text { "nero neronis }
\end{aligned}
$$

Catopsilia crocale $q$ f. latilimbata

Saletara panda f. nivaria

Anaphaeis java f. magniplaga

Terias tilaha myria

$$
\text { adustata }
$$

laeta naukratis

" " f. vimala

andesoni undana

lacteola varga

drona $f$. herlina
Formosa

Nausalaut

Tenimber

Obi

Upp. Burma

Annam

Siam

N. Borneo

Batu

Lombok

Sumatra

Assam

Mollucca

Sikkim

Indo-China

Yule Is.

Sumbawa

Lomblen

Sumbawa

E. Java.

Lombok

Kangean

Lombok

Bali

S. Java

E. Java

E. Java

E. Java

E. Java

W. Java

E. Java

E. Java

E. Java

E. Java

E. Java

W. Java

Kangean

W. Java

S. Java

S. E. Java

Bali

$\sigma^{7}$ E. Java, $q$ S. Java

W. Java

W. Java

W. Java

- W. Java, - S. Java

E. Java

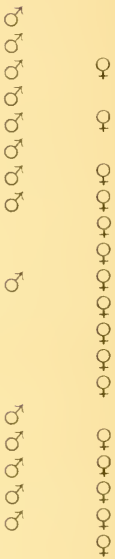

ช

q

ర

q

o

$0^{\pi}$

$0^{x}$

$0^{x}$

Q

Q

P

Q

Q

ㅇ

$\sigma^{2}$

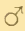

q

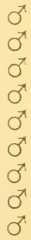




\section{Pieridae (Continued)}

Gandaca harina deformata

Pareronia valeria $f$. aureivena
$n$
,
") leona
" f. priventina
S. Java
E. Java
W. Java
W. Java

\section{DANAIDAE}

Danaida melissa f. myrsilos

$$
\begin{aligned}
& \text { albata gilva } \\
& " \text { adustata } \\
& \text { " similis vulgaroides } \\
& \text { luzonensis praemacaristis } \\
& \text { luzonensis simonides } \\
& \text { luzonensis panaitius }
\end{aligned}
$$

Hestia logani mevania
o E. Java, T W. Java
E. Java
W. Sumatra
E. Java
N. Borneo
Palawan

\begin{tabular}{|c|c|}
\hline " & " eurykleia \\
\hline$n$ & f. privata \\
\hline " & Of. eyrnia \\
\hline$n$ & f. biplagiata \\
\hline " & f. praestabilis \\
\hline " & Of. honrathi \\
\hline$"$ & wallengreni $f$. demaculata \\
\hline$n$ & climena terissa \\
\hline$n$ & deheeri lanios \\
\hline ” & crameri tenggerensis \\
\hline " & malayica hypenis \\
\hline ") & lacordairei $f$. decorata \\
\hline$n$ & " f. eleusinida \\
\hline$n$ & lombokiana \\
\hline$n$ & baweana \\
\hline$"$ & leucostictos f. abrupta \\
\hline » & f. radiata \\
\hline " & f. bioculata \\
\hline$"$ & f. demaculata \\
\hline$”$ & basilissa $\mathrm{f}$. donada \\
\hline
\end{tabular}
Lombok
S. Java

\section{EUPLOEINAE}

Euploea callithoe aroa
Rossel Is.

Fergusson

Misol

N. Guinea

Dorey

"Germ. ", N. Guinea

"Germ. ", N. Guinea

E. Java

Java

E. Java

E. Java

Java

E. Java

E. Java

Lombok

Bawean

W. Java

Ø̃ E. Java, \& W. Java

S. Java

W. Java

S. Java 


\section{NYMPHALIDAE}

Cethosia hypsea munjava

biblis alceste

1) $"$ nacoleia

hypsea fruhstorferi Stich.

penthesilea $f$. aelgitha

Ergolis merione nicevillei

1) isaeus lysias

" " munippus

Cupha erymanthus synnara

Cynthia crota varna

" " " javarna

Euthalia julii irina

$$
\begin{array}{ccc}
\text { " aconthea f. sacoida } \\
\text { " } & n \quad \text { f. arata } \\
\text { " } & \text { adonia culminicola }
\end{array}
$$

Tanaecia trigerta dandapani

Cirrochroa moeris $f$. oreta

Symbrenthia hypselis redesilla

Precis ida f. pseudiphita

Vanessa canace javanica

Yoma vasuki f. javana

" sabina f. atomaria

Hypolimnas anomala $\mathrm{f}$. violaria

$$
\begin{array}{lll}
\text { " } & \text { " } & \text { f. nivas } \\
\text { " } & \text { " } & \text { circumscripta } \\
\text { " } & \text { bolina } & \text { f. aphrodite }
\end{array}
$$

Doleschallia polibete $f$. polibetina

Lebadea alankara samana

Limenitis procris neutra

Rahinda hordonia pardus

Neptis vikasi taimiri

$$
\begin{aligned}
& \text { anjana zenca } \\
& \text { columella kangeana } \\
& \text { nata aletophone } \\
& \text { nandina phrasylas } \\
& \text { " apharea } \\
& \text { " ila } \\
& \text { " lizana } \\
& \text { " solygera }
\end{aligned}
$$

mindorana karpasa

chinensis $f$. areus

ananta taiwana

yerberyi dumicola

mahendra reducta

parthaca f. somula

$$
\text { ") }
$$

nandina ? junundiora

Pantoporia pravia caprotina
W. Java

Bawean

S. Java

E. Java

W. Java

W. Java

S. Java

S. Bornèo

ot W. Java, $q$ S. Java

S. Java

S. Java

W. Java

Bawean

E. Java

S. Java

W. Java

ơ E. Java, Q S. Java

W. Java

E. Java

W. Java

Java

E. Java

E. Java

S. Java

W. Java

E. Java

W. Java

E. Java

o E. Java, Q S. Java

W. Java

o W. Java, Q S. Java

O' E. Java, + S. Java

W. Java

Kangean

W. Java

ơ E. Java, Q S. Java

Sumatra

N. Borneo

Bazilan

Jolo

Palawan

Siao-Lou

Formosa

Tze-Tchuan

Formosa

Palawan

")

Lombok

E. Java 


\section{Nymphalidae (Continued)}

Pantoporia asura latecincta

Adolias dirtea javana

Herona sumatrana pringondani

Helcyra hemina masinia

Dicorrhagia nasimachus mannus

Euripus halitherses javanus

\section{f. lugubris \\ Q f. halizona \\ Q f. albostriata \\ Q f. danisepa}

Apatura nakula $q$ f. camiboides

$$
\begin{aligned}
& \text { " } \quad \text { " } \text { f. nilka } \\
& \text { " } \quad \text { f. nakuliana } \\
& \text { " parisatis javana }
\end{aligned}
$$

Prothoe francki irma

$$
\text { " " borneensis }
$$

Ergolis merione pharis

$$
\begin{aligned}
& \text { "1 ginosa } \\
& \text { 1) crestonia }
\end{aligned}
$$

specularia intermedia

$$
\begin{aligned}
& \text { dongalae arca } \\
& \text { phemonoe } \\
& \text { isaeus pupillata }
\end{aligned}
$$

Eurytela castelnau ochus

$$
\begin{array}{cl}
\text { " } & \text { ottonis } \\
\text { horsfieldi } & \text { senta } \\
\text { n } & \text { velitra }
\end{array}
$$

Melitaea cynthia leonhardi

$$
\begin{aligned}
& \text { " helles } \\
& \text { maturna idumides } \\
& \text { aurelia luceria } \\
& \text { " pharicia } \\
& \text { " valsunga } \\
& \text { i) marussia } \\
& \text { arduinna } \mathrm{f} \text {. rhodopensis }
\end{aligned}
$$

Argynnis pales palustris

$$
\text { selene lucotia }
$$

Prothoe australis prasia

$$
\text { " " . menodoo }
$$

Eriboea richthofeni

$$
\text { f. arana }
$$

pyrrhus babbericus

$$
\text { romanus }
$$

hebe kangeanus

$$
\text { " baweanicus }
$$

W. Java

S. Java

S. Java

or S. Java, \& W. Java

W. Java

W. Java

E. Java

W. Java

W. Java

W. Java

E. Java

W. Java

E. Java

W. Java

W. Sumatra

N. Borneo

Siam

o Malacca, $Q$ Sumatra

Palawan

Alor

Siam

C. Celebes

Wetter

Nias

S. Borneo

Palawan

Nias

Nias

Sumatra

Bulgaria

Sajan

Bosnia

Cogne

Krain

Barmsee

Saratow

Istria

Simplon

Ta-Tsien-Lou

Japan

Dutch N. Guinea

Waigeu

Tsingtoe

Babber

Roma

Kangean

Bawean

Lombok 


\section{Nymphalidae (Continued)}

Eriboea dolon carolus

eudamippus jamblidus

nepenthes fugator

nepenthes archaeus

delphus othonus

narceus megaduta

jalysus tryphonius

aristophanes

schreiberı valesius

pyrrhus pyrrhulus

dehaani Dbl. (Neallotype)

delphus cygnus R. \& J. (Neallotype)

Charaxes orilus kissericus

polyxenes varenius

distanti thespius

phlegmone

Sia-Lou

Lower Burma

Indo-China

Canton

Nias

Formosa

N. Borneo

Shortland Is.

W. Sumatra

Wetter

W. Java

E. Java

Kisser

Bawean

S. Borneo

W. Sumatra

\section{S. America}

Agrias amydon trajanus

$$
\text { ") "fernandi }
$$

Adelpha valentiana

$$
\begin{array}{ll}
\text { " } & \text { gavina } \\
\text { " } & \text { pleasaure antoniae }
\end{array}
$$

Prepona lygia

$$
\begin{gathered}
\text { eugenes decorata } \\
\text { " argyria } \\
\text { " transiens } \\
\text { pylene santina } \\
\text { " bahiana } \\
\text { " perischion } \\
\text { gnorima philetas }
\end{gathered}
$$

Pseudacraea pharsa
Obidos

Bahia

Rio Negro

Blumenau

Rio Grande

Blumenau

Chiriqui

Minas Geraes

Bahia

Theresopolis

Brazil

Bahia

Brazil

Honduraz

\section{Africa}

E. Africa

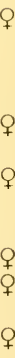

운

AMATHUSIIDAE

Zeuxidia dohrni

$$
\begin{aligned}
& \text { amythaon lurida } \\
& \text { otacilia }
\end{aligned}
$$

Amathusia phidippus baweanicus

$$
\text { majada }
$$

perakana taenia

phidippus f. retrograda

Thaumantis odana vedana

Clerome arcesilaus cyme
W. Java

W. Sumatra

S. Borneo

Bawean

E. Java

o S. Java, q W. Java

o $\mathrm{W}$. Java, $q$ Batavia

o $E$. Java, $Q$ S. Java

E. Java 


\section{Amathusiidae (Continued)}

Hyantis hodeva emarginata

Morphopsis biakensis derrhion

Morphotaenaris schonbergi athanael

Taenaris kubaryi $f$. eugenia

f. cyclopides

chionides aroana

kirschi verbeeki

1) anephoria

artemis humboldti

") blandina

electra

honrathi $f$. abdon

onolaus enomia

" macrophtalma

agrippa f. prodiga

rothschildi ansuna

rafaela

gisela $f$. timesides

Stichophthalma howqua miyana

tonkiniana

formosana
Dutch N. Guinea

Dutch N. Guinea

Bongu

"Germ. "N. Guinea

Germ. "N. Guinea

Aroa

Milne Bay

Yule Is.

Humboldt Bay

Jobi

Fergusson

Waigeu

Collingwood Bay

Jobi

Obi

Jobi

Jobi

Waigeu

Canton

Tonkin

Formosa

\section{BRASSOLIDAE}

Opsiphanes batea glaukias

" euchlaena

") praegrandis

() subsericea

catharinae gainas

cassina periphates

" munaties

tamarindi xiphos

sikyon

fruhstorferi Rob.

Caligo atreus dionysos
Brazil

S. Paulo

Paraguay

Rio Grande

S. Paulo

Colombia

Colombia

Venezuela

ơ W. Mexico, \& Honduras

Brazil

Chiriqui
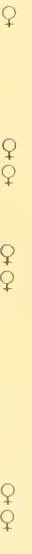

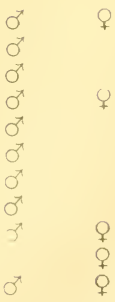

\section{MORPHIDAE}

Morpho anaxibia calliphon

$$
\begin{aligned}
& \text { " pelias } \\
& \text { perseus richardus } \\
& \text { didius julanthiscus } \\
& \text { aega bisanthe } \\
& \text { n mellinia }
\end{aligned}
$$

Parana

Rio Grande

Minas Geraes

Ecuador

Brazil

Espirito Santo 


\section{ELYMNIINAE}

Elymnias dusara f. dulcibella

$$
\text { f. coanescens }
$$

nessaea hermia

hypermnestra perpusilla

casiphone $f$. alumna

komara paruploea

i) f. pseudalma

dara bengena

ceryx hestinia

KUNSTLERI GAUROIDES
S. Java

S. Java

E. Java

$\sigma$ W. Java, + S. Java

S. Java

? Java

S. Java

o' S Java, $q$ W. Java

W. Java

Java: Tjisewu 16. Xl. $91.2000 \mathrm{~m}$

SATYRIDAE (Oriental)

Mycalesis sudra volsina

$$
\begin{aligned}
& \text { " tannis } \\
& \text { perseis prusias } \\
& \text { mineus macromalayana }
\end{aligned}
$$

Melanitis leda $f$. larrima

$$
\text { " } f \text {. ismenides }
$$

phaedima $f$. fulvinotata

zitenius ambasara $f$. griscesens

Erites argentina fruhstorferi Martin.

Ypthima iarbia eupeithes

$$
\begin{array}{ll}
\text { " } & \text { " gangamela } \\
\text { " } & \text { baldus nikias } \\
\text { " } & \text { pasitelides } \\
\text { " nigricans f. phasis } \\
\text { " pandocus andokides } \\
\text { " } & \text { f. otynia }
\end{array}
$$

Lethe dyrta anunda

$$
\begin{gathered}
\text { manthara } f \text {. symessa } \\
\text { " gemisia }
\end{gathered}
$$

chandica marga
E. Java

Bali

E. Java

Bawean

W. Java

W. Java

W. Java

S. Java

S. Java

W. Java

S. Java

Bali

Bawean

E. Java

E. Java

W. Java

S. Java

E. Java

E. Java

W. Java 


\section{Satyridae Palaearctic (Continued)}

Erebia evias eurykleia

" ottonis (- pyrenaica Stgr.)
" venaissina

oene philiata

n) pacula

") nilas

n) seliza

1) melara

1. eugina

7) vetulonia

" bonisa

" zagora

tyndarus agnitania

aethiops cosinida

$$
\begin{aligned}
& \text { " f. leucothenia } \\
& \text { " rubria } \\
& \text { " altivaga }
\end{aligned}
$$

Coenonympha arcania triumphans

$$
\begin{aligned}
\text { " } & \text { saleviana } \\
\text { " } & \text { chrysoaspida } \\
\text { " } & \text { mylestrei } \\
\text { jellacia } &
\end{aligned}
$$

Epinephele maera atabyris

herdonea

lenocinia
Martigny

Hautes Pyrenées

Mont Ventoux

Eithal

Bosnia

Champery

San Bernhardino

Racconaltal

Tournalle

Wetti

Bosnia

Bulgaria

Alpes-Mlaritimes.

ơ Wallis, O Champery.

Saleve

Mesocco

万ొ Col Golese, $q$ Garmisch

Bayern

Savoy

Saratow

Alpes-Maritimes

Klausen

Campement

Rhodos

of Saleve, $q$ Savoy

$\sigma$ Courmajeur, $q$ Simplon

\section{LIBYTHEINAE}

Libythea myrrha yana

E. Java

\section{ERYCINIDAE}

Sriboges, calycoides

Dodona eugenes venox

adonira argentia

ouida phlegra

elvira $F$ f. pura

durga rubula

WINDU

fruhstorferi Rôb.

Abisara kansanbi geza " erilda

)

kansanboides tina
W. Java

of Bhutan, Q Assam

Upp. Burma

Moussuri

N. Borneo

hinutschan

Java

W. Java

o S. Java, $q$ E. Java

S. Java

$\sigma^{\top}$ W. Java, q S. Java 


\section{LYCAENIDAE}

Holochila intensa ernita

dimorpha decolorata

ilias kiriwina

obiana

gelnua

Epimastidia staudingeri fergussonia

werneri

kapaura

speiron speironides

schaeffera annamensis

soranus

Thysonotis coelius synesius

$$
\begin{array}{cc}
" & \text { aetius } \\
\text { " } & \text { vesalius } \\
\text { cyanea coroncia } & \text { obiana } \\
" \quad \text { cordella } \\
\text { " arinides } \\
\text { peri valestinax } \\
\text { " oribasis } \\
\text { wallacei metrophanes }
\end{array}
$$

Poritia hewitsoni trishna

$$
\begin{aligned}
& \text { n n } \quad \text { f. palilia } \\
& \text { erycinoides psophis } \\
& \text { philota taimana } \\
& \text { ") nicias } \\
& \text { sumatrae milia } \\
& \text { pheretia bilitis } \\
& \text { proxima chimara } \\
& \text { erycinoides -. f. coronata } \\
& \text { f. demaculata } \\
& \text { naukydes f. nigra } \\
& \text { javanica } f \text {. abisurina }
\end{aligned}
$$

Lampides aratus $f$ gamadia

$$
\text { philatus athanetus }
$$$$
\text { parasatura }
$$

daoinides Rôb.

kankena metallica

kondulana sydra

$$
\text { piepersi }
$$

Jamides nila $f$. nilana

Castalius roxus astapus

$$
\text { " hamada nivata }
$$

Lycaenopsis cardia astarga

f. paradilecta

catreus hereias

marginata carinita

Spindasis syama rectilineata
Waigeu

Kapaur

Kiriwina

Obi

"Germ. ") N. Guinea

Fergusson

"Germ. "N. Guinea

Kapaur

Germ. "N. Guinea

Annam

Halmaheira

$\mathrm{Obj}$

"Germ. " N. Guinea

Dutch N. Guinea

N. Hannover

Obi

Halmaheira

Brit. N. Gitinea

ơ" N. Guinea ", Q Kapaur

Dutch N. Guinea

o' Sorong, \& Dorey

Assam

Sikkim

N. E. Sumatra

Nias

N. Borneo

Sintang

N. E. Sumatra

Sintang

S. Java

S. Java

W. Java

W. Java

S. Java

S. Java

o W. Java, $Q$ E. Java

W. Java

E. Java

E. Java

Java

S. Java

S. Java

W. Java

W. Java

W. Java

S. Java

W. Java

E. Java 


\section{Lycaenidae (Continued)}

Curetis santana $f$. subobsoma

$$
\text { 1) baweana }
$$$$
\text { " bazilana }
$$

Sithon nedymond chitra " thesmia vanica

Bindahara phocides f. phocidiana

Semanda superba gloriosa

Tajuria mantra mesambria

" cleoboides epigenes

" isaeus pisatis

n plataia

Dacalana vidua sinhara

Tajuria jangala $f$. hella

Marmessus medullia

Araotes lapithis archytas

$$
\text { " " decolor }
$$

Sinthusa malika volsa

$$
\text { " nasaka obscurata }
$$

" amata

Zeltus etolus prupadius

Deramas livens livescens

Yasoda pita f. emana

"singama atymnus deinostratus

$$
\text { " matienus }
$$

Nacaduba emolus javanus pernsia agorda 1) baweanana

bernice isana

bernice icena glauca Snell. datarica Snell. pavana lysa

$$
\text { " bajava }
$$$$
\text { 1) minya }
$$

Niphanda fusca syme

Arhopala malayica fundania apha apharida fulgida tenea adorea gorgias morphina sidicina hannon bazalus ambivius anthelus jabadia azata pangeran bazalus pratinas amantes aphobius diardi asatha agnis hagius
W. Java

Bawean

Bazilan

ơ S. Java, 9 E. Java

S. Borneo

W. Java

ơ S. Java, + W. Java

W. Java

W. Java

W. Java

S. Celebes

N. E. Sumatra

S. Java

or S. Java, Q E. Java

S. Java

Nias

S. Java

Sikkim

Sumatra

W. Java

W. Java

W. Java

S. Java

W. Java

S. Java

S. Java

W. Java

Bawean

W. Java

Sintang

E. Java

W. Java

N. E. Sumatra

Siam

Lombok

W. Java

E. Java

W. Java

S. Java

W. Java

N. E. Sumatra

W. Java

S. Java

W. Java

W. Java

W. Java

S. Java

W. Java

S. Java 


\section{Lycaenidae (Continued)}

Arhopala fruhstorferi Rôb (=aedias Hew.)

$$
\begin{aligned}
& \text { apidanus antipaxus } \\
& \text { sandakani aytonia }
\end{aligned}
$$

Deudorix epijarbas cinnabarus $q f$. side " jarbas dikeiarchus
"
n n
f. yabala

Rapala carthema liturnia

$$
\begin{array}{ll}
\text { " } & \text { nissa odosia } \\
\text { " } & \text { manea asikana } \\
\text { " } & \text { varuna zulkarna } \\
\text { " } & \text { pheretina sakaia } \\
\text { " } & \text { sagasa } \\
\text { " } & \text { schistacea celuta } \\
\text { ") } & \text { " renata }
\end{array}
$$

Mahathala ameria javana

Gerydus symethus perlucidus

$$
\text { " boisduvali oxylus }
$$

Allotinus strigatus dositheus

$$
\text { " posidion }
$$

Logania marmorata javanica

$$
\text { 1) massalia alypha }
$$

Megisba hylax corax

Chrysophanus virgaureae pelusiota

$$
\text { osthelderi }
$$

theages

of f. seriata

f. fredegunda

б. galsintha

juvara

athanagild

cissites

anaphidamas phintonis

tamalana banghaasi

hippothoe cisalpina
W. Java

E. Java

W. Java

S. Java

W. Java

S. Java

S. Java

W. Java

W. Java

S. Java

S. Java

Bawean

W. Java

б. S. Java, + E. Java

C. E. Java, Q S. Java

E. Java

E. Java

W. Java

W. Java

o S. Java, $q$ W. Java

W. Java

E. Java

E. Java

S. Java

ơ Cogne, $q$ Piedmont

o Formayztal, Iselle

Brusio

Zermatt

Zermatt

Alton

Passau

Engadine

Etflatal

Irkutsk

Chantengri (Kuldacha)

or Alpe Veglia, \& Fusio 


\section{HESPERIDAE}

Hasora badra madatta

Telicota rectifasciata sakka

$$
\begin{aligned}
& \text { chariyawa } \\
& \text { dara tanya } \\
& \text { yojana } \\
& \text { paragola }
\end{aligned}
$$

Taractrocera ikramana

Plastingia naga valinia

$$
\begin{aligned}
& \text { " " pellonia } \\
& \text { corista patmapana }
\end{aligned}
$$

Tagiades trichoneura nava

gana avala atticus tubulus

Odontoptilum mahabini

$$
\text { " pygela javanica }
$$

Koruthaialos rubecula haraka

Gangara thyrsis pandia

Halpe zema vistara

Acerbas antheas tagiadoides

Oeranea neaera pusilla

Lophoides pelethronia

Satarupa affinis javana

dirae visana

Daimio adrastus meluchus

Notocrypta singularis

$$
\begin{aligned}
& \text { fraga } \\
& \text { avattana } \\
& \text { sidha } \\
& \text { alysos f. asanga }
\end{aligned}
$$

Coladaenia dan eacus

Celaenorrhinus maculipennis piepersi

$$
\text { simula binotatus }
$$

Pirdana hyela scanda
Java

W. Java

S. Java

o' W. Java, f S. Java

S. Java

S. Java

S. Java

S. Java

W. Java

W. Java

o' W. Java, \& S. Java

S. Java

W. Java

W. Java

W. Java

W. Java

W. Java

S. Java

$\sigma^{x}$ S. Java, $q$ E. Java

Java

W. Java

W. Java

W. Java

W. Java

W. Java

$\mathrm{O}^{x}$ S. Java, $q \mathrm{~W}$. Java

W. Java

S. Java

S. Java

S. Java

W. Java

S. Java

o E. Java, \& S. Java

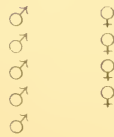





\title{
SOME NOTES RESRECTING TYPES
}

The possession of the types of the Fruhstorfer Collection and the series of specimens from which the original description were made is of the greatest importance to all students of butterflies in the future.

Mr. Fruhstorfer has described such a large number of forms from little-known places of which the fauna is very poorly represented in most collections in Europe, that it is a very considerable asset to have this types available for study by independent students.

A very great number of specimens labelled "type" are not entered in the type Catalogue, because they bear no name label.

They represent probably for the most part doubtfully distinct forms. In some cases more than one $\sigma^{\prime}$ and one $q$ are labelled "type". The selection of a holotype and an allotype is left to some future student. In many other cases one sex only is labelled "type" when the other sex is also present.

It is uncertain whether this sex was undescribed or whether the type label was omitted. Reference must be made to the original description.

In the list of types we have given the indication where one specimen of one or both sexes only exists. Whether the name was founded on more specimens which have since been dispersed can only be ascertained by reference to the original description.

Where no mention is made by the author of his original series, we understand that it may be assumed that he had before him only those specimens now contained in the collection.

A few types described by Rober are also contained in the collection.

Georges TALBOT

\section{Copy of a letter from Mr. Fruhstorfer to the Director of the Hill Museum, Witley Surrey}

\author{
Lugano, 18, ill, 19.
}

What concerns my types, there is an irregularity in fixing those. The first years, shall we say up to 1899 , I considered as types all the specimens before me and I fixed a label "type " sometimes under a dozen of specimens, and sold also some of them. Later on, I learned to understand that there was only one type or two, if 1 had $\sigma^{\top}$ and $q$ before me and 1 attached the type label only to one, or if $\mathrm{I}$ had a couple to two specimens. The case with DELIAS battana is very peculiar.

The specimen figured was than treated by me as type. The specimen is the one now in my collection. Of the $q Q$ there are two forms - a white and a yellow one. 1 did not attach a label to them, but I never sold a true type out of the collection of Indo Australians. The specimens in the Paris Museum are not types as 1 treated them since 1900. Of course the number of species and races described before 1900 is very limited. Most of my descriptions appeared after 1905 as soon as I began to retire from business.

But as a rule you have not to fear confusion. Everything inside my collection has to ve treated as a real type, everything outside as a cotype. So for i. the types of Delias from Lombock are only in my collection. " 



\section{THE IRUASTORER COLLECTION}

of Burterffies

\section{GENERAL ACCOUNT}

with List of the more interesting forms.

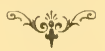





\title{
BUTTERFLY COLLECTING and the FRUHSTORFER COLLECTION
}

\author{
By GEORGES TALBOT, F. E. S.
}

Since the time when Man began to amass collections of objects in Nature and Art. Butterflies have alway's found a place, and to-day there is probably as many collectors of these insects, as there are of stamps.

The passion for Beauty has inspired the butterfly-collector to take up this hobby. It begins mostly in early years; with many it becomes a study; with some this study adds largely to scientific knowledge.

The importance of large collections of Butterflies and Moths is now recognised, not alone for the elucidation of purely scientific facts of Natural History, but as an aid to the investigation of larger problems and of the economic questions which face the agriculturist whose crops are destroyed.

The Collection of Butterflies brought together by the late H. FRUHSTORFER has already provided that scientist with much material for study, resulting in a prolific output of writing.

The published writings of Fruhstorfer, though dealing mostly with systematics, will be valuable to the worker of the futur, especially in regard to the distribution and variation of the insects dealt with.

In the graet work edited by Seitz, "The Butterflies and Moths of the World", the studies of Fruhstorfer, based on his wonderful collection, are largely evident, and thus a rich legacy of information is made available to entomologists the world over.

This published work of Fruhstorfer was only possible by assiduously and intelligently collecting material.

To this end he made many journeys, principally in the tropics of the East, visiting India, China and most of the Malayan Islands.

The journeys made by the Butterfly Hunter are not picnics, particularly, when the region visited is little known to travellers

All the discomforts of disease, hunger, thirst and dangers from wild animals, noxious insects and savages, enter more or less into the daily existence of the searcher after rare butterflies.

Many of the best known species are obtained only after considerable expense and difficulty. With all this, the enthusiastic entomologist finds always his recompense when the long expected capture:s are made.

The uninitiated are apt to overlook the enormous amount of work and perseverance necessary to bring together a really fine collection of Butterflies by individual effort.

We have seen that the getting of the species is no easy matter. They have next to be set up for the cabinet, and most important of all, to be properly labelled with the locality. date and other information according the capture.

Where large series of specimens are obtained it is necessary to weed out a good many. The value of a Collection may often depend upon how this weeding out is done, for impor tant though insignificent individua' variations may be overlooked.

The variation to which butterflies are subject, is so remarkable that many species are only adequately represented by a large series of specimens.

It is gratifying to observe that this important factor of variation has been closely attended to by Fruhstorfer in building up his collection. 
We may-be sure that no slight variation has been discarted. From this point of view, the Collection possesses an added value.

Again, the Collection is rich in Types, i. e. specimens which are the originals from which descriptions were made of forms new to science.

Although the Collection is largely representative of the Eastern Tropics, there are typical examples of forms found in all other parts of the world, including Europe.

If we compare a Collection of this kind with one of pictures or of antiquities, we find that it possesses peculiar features which render it the equal of the former and the superior of the later.

The great evolutionary process is reflected in the butterfly wing and the "pictures" or patterns displayed are full of meaning to those who learn to understand them.

To the uninitiated, these patterns are no more than the symbolic writing on the cases of the Pharoahs.

Yet these markings on the butterfly wing afford the scientist valuable clues to his understanding little by little, some of the inner secrets of Nature, of the more recondite problems presented by the universe of living matter.

All the wonders of Butterfly patterns are seen in this great Collection and we are introduced to colours which no artist can faithfully imitate.

We may mention the gorgeous metallic blues of all shades which are displayed by the American Morphos, the rich velvety black and gold and green of the large Ornithoptera of the Malayan lslands, and the wonderful opalescence and iridescence shown in some Erycinidae of America and by the Satyrid, Lamprolenis nitida, of New Guinea.

We are introduced to the smallest known species among the family of the "Blues", and to the most gigantic forms among the Ornithoptera. We find also some kinds which Ay at 15,000 to 18,000 feet in the Himalayas, and others which brave the icy barriers of Groenland and Arctic America.

In conclusion, we must express the hope that this wonderful collection will find one lay a place in a Museum, where it may enrich the series and provide additional date for the study of the various problems which insects present. 


\section{Copy of the First Report on the FRUHSTORFER COLLECTION By GEORGE TALBOT \\ F. E. S. Curaior of the HILL MUSEUM of LEPIDOPTERA, WITLEY, Surrey ENGLAND}

I have examined the Collection of Butter flies known as the

\section{FRUHSTORFER COLLECTION}

and 1 testify that it is thoroughly representative of the Indo-Australian and Palaearctic Faunas, besides being rich in the South American groups.

IT IS CERTAINLY ONE OF THE FINEST COLLECTIONS EVER BROUGHT TOGETHER OF THE INDO-MALAYAN FAUNA and a great part of it was collected on the field by late Mr. Fruhstorfer himself

The principal Localities represented are:

India, Burmah, Tenasserim, Perah, Malay Peninsula, Tonkin, Annam, Siam, South China, Formosa, Surinam, Liu-Kiu Islands, Mindoro, Mindanao, Bazilan, Palawan, North and South Celebes, Bangkay, Sula ls., Saleyer, Buton, Tanah Djampea, Engano, North and South Borneo, Natuna ls., Java, Bawean, Bali, Lombok, Sumatra (especially the Battah), Nias, Batu. Banda, Sumbawa, Sumba, Timor, Obi, Batjan, Halmaheira, Buru, Ceram, Amboina, Saparua, Wetter, Alor, Kisser, Dammer, Tenimber, Key, Aru, Flores, Mysol, Waigeu, Salawatti, the Coast districts of New Guinea (and especially the German territories), Milne Bay, Fergusson lsl., Yule, Bismarck lsl., Salomons, Fidji, Palu, North Australia.

The Island. of Java is very thoroughly represented by about 5.000 Specimens of which we give details elsewhere. THIS INCLUDES MANY OF THE GREATEST RARETIES, WHICH HAVE EITHER NOT BEEN FOUND SINCE OR WHICH EXIST ONLY IN FEW EUROPEAN COLLECTIONS.

With few exceptions, all the specimens are in very fine condition, well set and properly labelled, all are named, with the exception of some doubtful forms which may represent new races.

The specimens are contained in :

\section{THIRTY 26 DRAWER CABINETS and TEN 48 DRAWER CABINETS}

There are more than 1,200 Drawers.

All Drawers are interchangeable and fitted with Glass Bottoms, they are in polished Lindenwood, the Cabinets in Walnutwood, all of the same style, with Pannel doors.

In the details given elsewhere, we have cited only a few of the most interesting species.

IT IS SAFE TO SAY, THAT ALL GROUPES ARE EXTREMELY WELL REPRESENTED and some idea of this may be gathered by reference to the work of Seitz "The Macrolepidoptera of the Globe".

It will be found, that with very few exceptions, the names given to the forms are correct and very few collecticns of this magnitude are so well named. 


\section{Contents of Cabinets}

1. Papilionidae. (1)

2. $n$ (2)

3. (3)

4. Pieridae, (1)

5. " (2)

6. Nymphalidae. (1) Delias.

$7 . \quad$ (2) Hypolimnas, Cethosia.

8. " (3) Euthalia, Adolias:

9. Troides. (1)

$10 . \quad$ (2)

11. Papilio (American) (African), large Cabinet.

12. Danaidae. (1) Thais, Teinopalpus.

13. (2)

14. Euploeinae. (1)

15. (2) Large cabinet.

16. Acraeidae, Nymphalidae (American). (1)

17. Nymphalidae (American). (2)

$18 . \quad$ " (3) Prepona.

19. Melitaea, Argynnis, Nymphalidae (Amer), cont Agrias.

20. Argynnis.

21. Pantoporia, Limentitis, Kallima

22. Charaxes, Nymphalidae (African).

23. Neptis,

24. Amathusiidae.

25. Taenaris, Morpho.

26. Morphidae, Brassolidae.

27. Elymnias, Mycalesis.

28. Satyridae.

29. " (Palaearctic.)

30. Erebia.

31. Satyridae (American) (Palaearctic).

32. Libythea, Erycinidae.

33. Lycaenidae. (1)

34. $\quad$ (2)

$35 . \quad$ (3)

36. 11 (4) (Palaearctic).

37. Heterocera.

38. Hesperidae.

39. Parnassius.

40. Java

41. "

42. , 


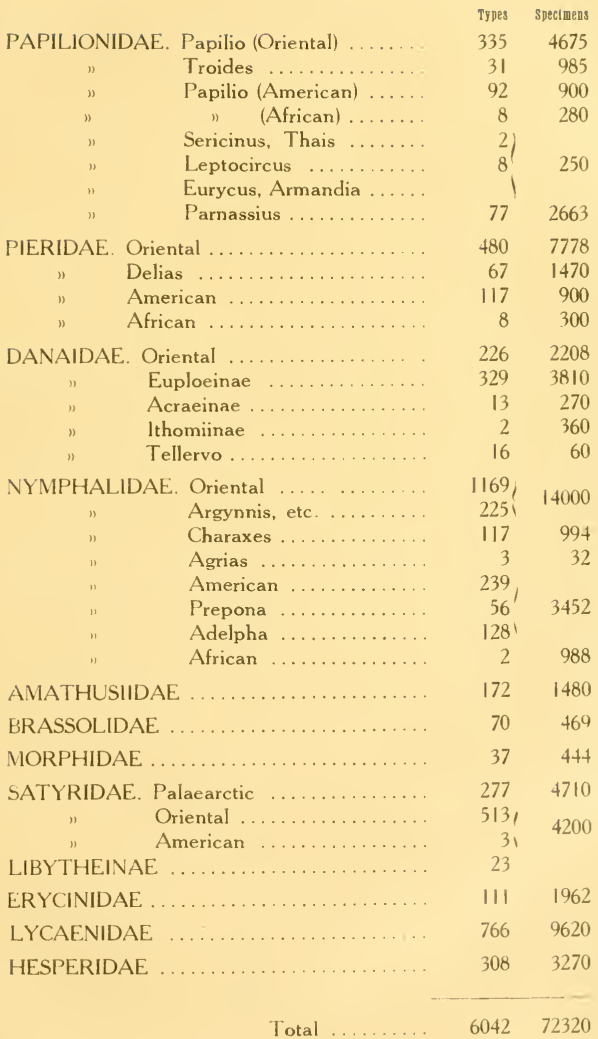

Approximate total of specimens, say .......... 70000

Total number of Types (one for each name) ...... . 6042

" " " " (including each sexe) ..... 8657 


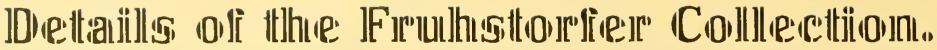

\section{PAPILIONIDAE}

Number of named forms represented by TYPES.

The following are included :

TROIDES paradisea

neomiranda

croesus

supremus

priamus

lydius

honrathiana

tithonus

iris

regis

nais

regina

sumbawana

victoriae form

dohertyi

bornemanni

miokensis

urvilleana

Number of specimens about.

PAPILIO gambrisius

bridgei

woodfordi

inopinatus

ahasverus

pitmani

canopus form

subfasciatus

rumanyovius form

memnon forms.

lampsacus

torbesi

ilioneus

pericles forms

montrouieri

adamantius

dorcus

atratrocles

hermocrates

aristinus

rhesus

leosthenes

euphrates

\begin{tabular}{|c|c|c|}
\hline 1s o & 1' $q Q$ & \\
\hline 10 & $1 Q$ & \\
\hline $9 \sigma^{x} \sigma^{x}$ & 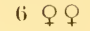 & \\
\hline $2 \sigma^{-1} \sigma^{x}$ & 19 & \\
\hline 200 & $4 q Q$ & \\
\hline 10 & $\because Q q$ & \\
\hline 200 & $3 q q$ & \\
\hline & 19 & (Waigeu) \\
\hline 18 & & \\
\hline 10 & 19 & \\
\hline $3 \sigma^{\top} \sigma^{\top}$ & $\because$ ? & \\
\hline & 19 & (Malacca) \\
\hline $2 \sigma^{\prime} \sigma^{\prime}$ & 10 & \\
\hline & 19 & (Salomons) \\
\hline 200 & $5+9$ & \\
\hline 10 & $1 Q$ & \\
\hline 10 & 19 & \\
\hline $10^{x}$ & $3 q+$ & \\
\hline
\end{tabular}

1000

( 30

5 ठั

" $\sigma^{x} \sigma^{x}$

$3 \sigma^{x} \sigma^{x}$

10

Series of

ช̆

5 б

$7 \sigma^{x}$

$60^{-1}$

$3 Q Q$

$7 \sigma^{x}$

15) $0^{7} 0$

10

10 $\sigma^{x} \sigma^{x}$

1 व

(i ฮั

10

$3 \sigma^{\circ}$ 
PAPILIONIDAE (Continued)

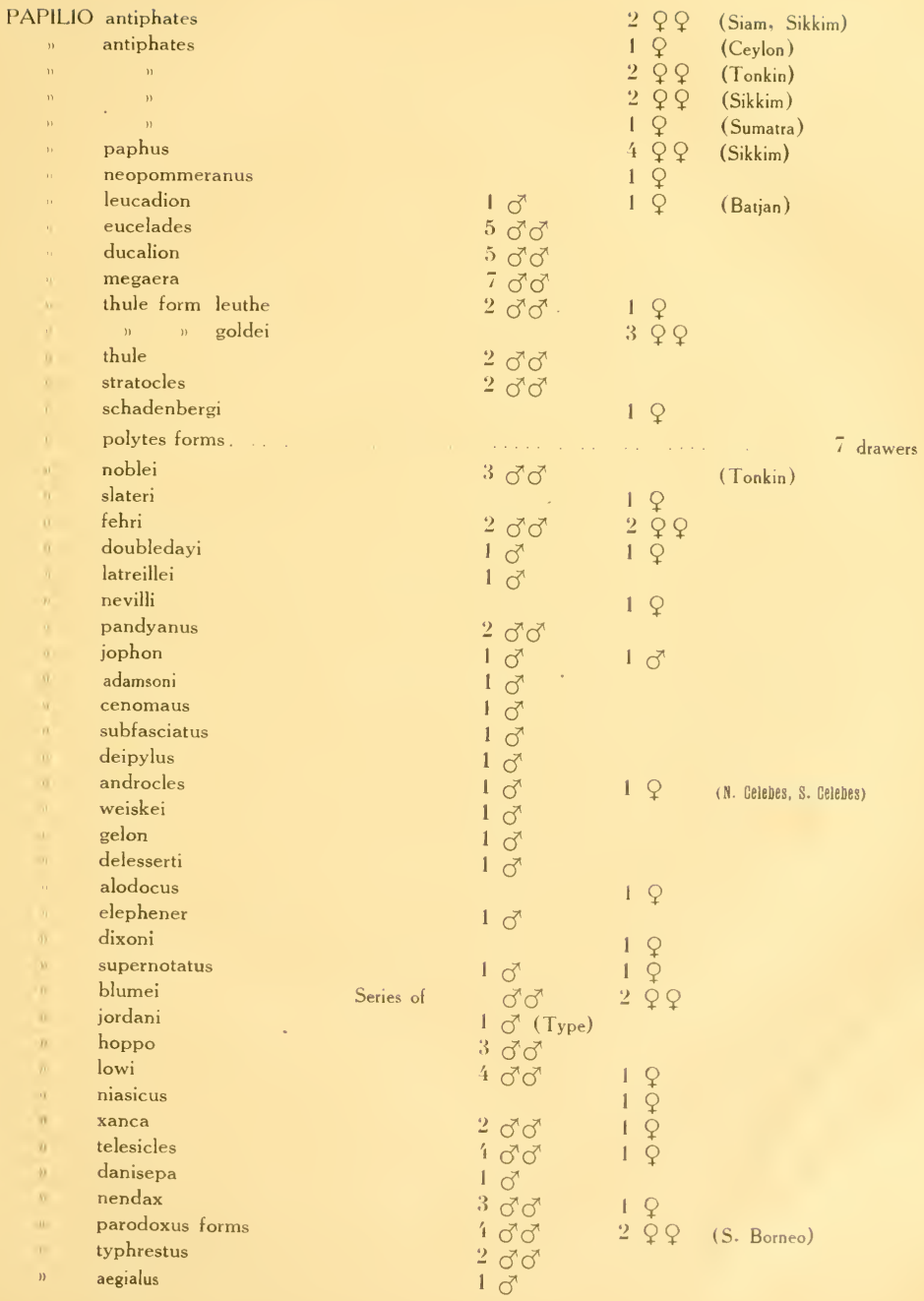




\section{PAPILIONIDAE (Continued)}

PAPILIO sticheli

$$
\begin{aligned}
& \text { oenigna } \\
& \text { telearchus } \\
& \text { liris oreon } \\
& \text { priapus } \\
& \text { paradoxus } \\
& \text { payeni } \\
& \text { brunei } \\
& \text { alcibiades } \\
& \text { prillwitzi }
\end{aligned}
$$

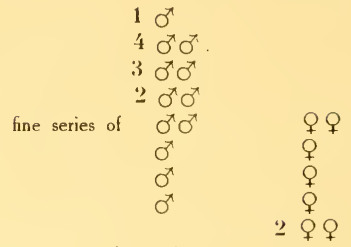

(Type, Unic)
LEPTOCIRCUS .... one drawer. . 6 Types TEINOPALPUS two drawers ARMANDIA lidderdali

SERICINUS
EURYCUS
LUEDORFIA
THAIS

I Type $4 \sigma^{x}$
$\sigma^{x}$
1 Type $\sigma^{x}$

2 Types

\section{PAPILIO (African)}

Including :

PAPILIO antimachus

" uganda

" dardanus (good forms)
100

$7 \sigma^{x} \sigma^{x}$ q

specimens about :

Number of specimens about....... 300

Forms represented by Types

\section{PAPILIO (American)}

Including :

PAPILIO erostratus

victorinus morelius

ascanias

xynias

hectorides $q$ form melania

helios

homerus $20^{x}$

$10^{\pi}$

$20^{x} 0^{x}$

$2 \sigma^{\pi} \quad 1$ ?

$2 \sigma^{x} \sigma^{x}$

$3 q q$

Number of specimens about. .

Forms represented by types.

\section{PARNASSIUS}

Number of specimens about

Form represented by types. 


\section{DELIAS}

Number of specimens about

Forms represented by types.

DELIAS chrysorhaea tobohana

villia

egialea horracki

parthenope

inferna

enniana

cinerascens

hageni

fruhstorferi

bornemanni

diva

kummeri

isocharis

itamputi

clathrata

iltis

cunningputi

microsticha

conversa

weiskei

phaeres

cathara

damala

callima

niepelti

indistincta

themis

chrysomelaena

philotis

candida

fasciata

isabellae

choiseuli

schœenbergi

palawanica

luzonensis

timorensis forms from

sacha

bagoe

salwini

poecila

edela

lytaea

bromo (a series)

battana

aurantiaca (series)

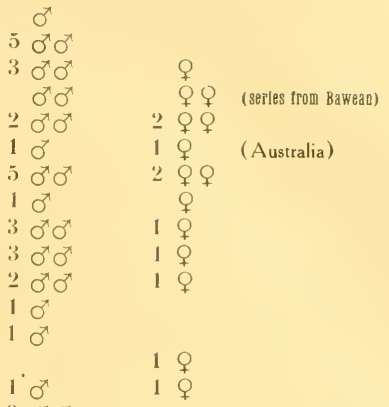

$2 \sigma^{x}$

ㄱ. $\sigma^{x} \sigma^{x}$

1 वे

$3 \sigma^{\pi}$

10

10

$2 \sigma^{x}$

$10^{x}$

$30^{\pi} \sigma^{\pi}$

$20^{x}$

$10^{x}$

- $0^{\pi} \sigma^{\pi}$

10

$1 \sigma^{x}$

$30^{x}$

$3 \sigma^{x}$

i $\sigma^{x} \sigma^{x}$

1 ठ

$30^{x}$

10

10

1 ठ

10

$20^{x} \sigma^{x}$

10

$2 \sigma^{x} \sigma^{\pi}$

$3 \sigma^{\pi}$

19

19

10

200

19

(Mindanao)

$\begin{array}{ll}1 & 9 \\ 1 & 9 \\ 3 & 9 \\ & 9 \\ 1 & 9 \\ 1 & 9 \\ 1 & 9 \\ 1 & 0\end{array}$

(Babber, Klsser, Walter Roma aod TenImbers. 
APPIAS. - Number of specimens about.

including fine female forms of nero and others.

HUPHINA - Number of specimens about. . . . . . . . . .

PIERIS. - Number of specimens about..............

including long series of Palaearctic forms

METAPORIA, etc.

EUCHLOE

Number of specimens about..... $\quad 90$

LEPTOSIA

about..... 70

ELODINA

about..... 90

APORIA

about..... 80

PRIONERIS

including :

about.....

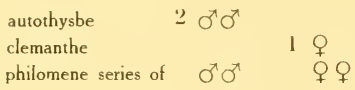

SYNCHLOE.

Number of specimens about.....

200

IXIAS

including :

Number of specimens about.....

\begin{tabular}{|c|c|}
\hline $\begin{array}{l}\text { malumpsinum } \\
\text { flavipennis } \\
\text { ludekingi }\end{array}$ & $10^{x}$ \\
\hline kühni & $10^{x}$ \\
\hline paluensis & $3 \sigma^{x} \sigma^{x}$ \\
\hline bagenstecheri & i $\sigma^{\top} \sigma^{x}$ \\
\hline venilla & \\
\hline
\end{tabular}

LEUCOPHASIA.

Number of specimens about. .

100

GONEPTERYX

HEBOMOIA

Including many females :

about. 170

about. . 18()

C.ATOPSILIA

Number of specimens about....

TERACOLUS

COLIAS

including :

about. .

sagartia

arida

regia

romanovi maculata

staudingeri pamira

$10^{x}$

10

$2 \sigma^{x} 1$

$10^{\pi} 19$

10

about. .

TERIAS. - Number of specimens about 
DANAIDA, etc... - Number of specimens about. including :

DANAIS

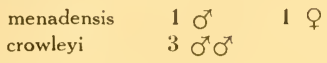

IDEOPSIS. - Number of specimens about. . . . . . . . . . . . . . . . . 250

NECTARIA \& HESTIA. - Number of specimens about. . . . . . $\quad 150$

Forms represented by types..................... 190

\section{EUPLOEINAE}

Number of specimens including many fine species about.........

Forms represented by types. . . . . . . . . . . . . .

ITHOMIINAE

Number of specimens about

\section{PALAEOTROPINAE}

TELLERVO one drawer

Forms represented by types.

\section{DANAIDAE}

DANAIDAE african, Number of specimens about.

ACTINOTE
ACRAEA

about.

about. . . . . . . . . . . . . . . . . 150

NYMPHALIDAE (Palaearctic \& Eastern)

MELITAEA.

ARGYNNIS

PANTOPORIA

AMNOSIA

CHARAXES
Number of specimens about.

1.050

about.

1. 750

about.

about...... 400

about......

including :

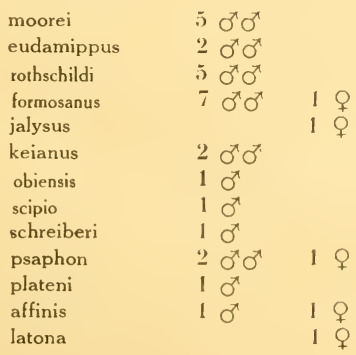


CHARAXES

$\begin{array}{llll}\begin{array}{l}\text { meridionalis } \\ \text { hannibal }\end{array} & 1 \sigma^{\pi} & \\ \begin{array}{l}\text { orilus wetterensis } \\ \text { nitebis }\end{array} & 1 \sigma^{\pi} & \\ \begin{array}{l}\text { caphontis } \\ \text { durnfordti everetti }\end{array} & 1 \sigma^{\pi} \sigma^{\pi} & 1 & q \\ \begin{array}{l}\text { connectens } \\ \text { staudingeri (series) }\end{array} & 3 \sigma^{\pi} \sigma^{\pi} & 4 & \\ \begin{array}{l}\text { amycus georgius } \\ \text { caroli }\end{array} & 1 \sigma^{\pi} \sigma^{\pi} & 1 & 1 \\ & 1 \sigma^{\pi} & 1 & q\end{array}$

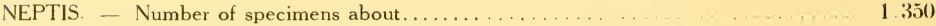

EROLIS " n about............

PENTHEMA \& CALINAGA - Number of specimens about. . . . . . . . . . . . . 80 including :

PENTHEMA annamitica dorlisa

CALINAGA lhatso

CETHOSIA - Number of specimens about. . . . . . . . . . . . . . . . 500

CYNTHIA

about. ......

CUPHA

about.....

CIRROCHROA - Number of specimens about.

ATELLA \& ISSORIA "

about. .

TERINOS

about.

PRECIS

about. .

VANESSA \& PYRAMEIS

about.....

including :

V. limenitoïdes $2 \sigma^{\nearrow}$

HYPOLIMNAS - Number of specimens about..

including about $300 \mathrm{H}$. bolina with fine series of female forms.

CYRESTIS. - Number of specimens about.

DOLESCHALLIA "

YOMA

about.....

RHINOPALPA

about

KALLIMA. - Number of specimens about. .

including fine paralecta series, herona prigondani series

SYMBRENTHIA. - Number of specimens about. . including
hippalus
$6 \sigma^{x}$
$2 q Q$

PARTHENOS. - Number of specimens about. . . . . . . . . . . . . . . . . . . . 200

HESTINA \& EURIPUS - Number of specimens about. . . . . . . . . . . . . . . . . 150 many very fine female forms.

HERONA \& EULACEURA. - Number of specimens about.

EUTHALIA \& TANAECIA - Number of specimens about. including

sikanid adonia 
ADOLIAS - Number of specimens about. including :
satrapes
10
tyrtacus
19

APATURINA - Number of specimens about.... . . . . . . . . 40

SEPHYSA

APATURA. -

$\begin{array}{ccc}" \prime & " & \text { about... } \\ \text { ncluding } & & \text { about... }\end{array}$

including

MYNES

nakula

parisatis and fine fe males.

PROTHOË

Number of specimens about.....

\begin{tabular}{|c|c|c|}
\hline calydonia & 9 $\sigma^{x} \sigma^{x}$ & $1 f$ \\
\hline semperi & $10^{\pi}$ & \\
\hline plateni & $10^{x}$ & \\
\hline mulderi & $1 \sigma^{x}$ & \\
\hline ribbei & $1 \sigma^{x}$ & 1 \\
\hline niasica & $4 \sigma^{x} \sigma^{x}$ & \\
\hline
\end{tabular}

about. ....

EURIPUS , 7 female forms

ERIBOEA pyrrhus antigonus

$$
\text { „) seitzi }
$$

scipio

gilolensis

dehaani

sultan

delphis cygnus

schreiberi niasicus

schreiberi

clitarchus

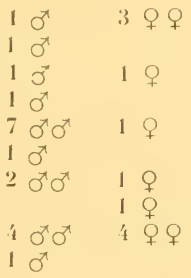

(Choiseul)

$$
\text { Number of specimens about. . }
$$

Number of specimens of NYMPHALIDAE about

NYMPHALIDAE (American)

Number of specimens about

Forms represented by types.

HELICONIUS \& EUCIDES. - Number of specimens about......

about......

Including : 
CALLITHEA

Including :

depuiseti

AGERONIA \& PERIDROMIA

CHLORIPPE

EUNICA

Including :

sydonia

erota

sophonisba

coelina

emmelina

ADELPHA

AGRIAS

Including :

trajanus (type)

fernandi (type)

aedon (type)

amydon forms (Perou)

claudina

lugina

lugens form

hades

Number of specimens about.....

$\because q Q$

Number of specimens about.... $\quad 200$

n $n \quad$ about.... 150

n 1 about.... 140

$q$
$q$
9
3
9
9
9
$q$

Number of specimens about.....

800

" " about.....

32

PREPONA.

Including :
xenagoras
deiphile

OTHER GENERA.

$\sigma^{x}$
$\sigma^{x}$
$\sigma^{x}$

Q

Number of specimens about.....

$5(j)$

1 운

19

Number of specimens about.

1000

NYMPHALIDAE (African)

Number of specimens about.

5()

\section{AMATHUSIIDAE}

AMATHUSIA, etc

Number of specimens about.

1. $(00)$ Including :

ZEUXIDIA pylaon

aurielus (series)

plateni

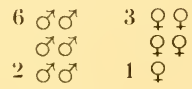

STICHOPHTHALMA

Number of specimens about .....

Including :

fruhstorferi

mathilda

louisa

$\begin{array}{ll}4 \sigma^{\pi} \sigma^{\pi} & 4 \text { q } \\ 2 \sigma^{\pi} \sigma^{x} & \\ 1 \sigma^{\pi} & 19\end{array}$

MORPHOTAENARIS

schoenbergi

athanael (type)

$2 \sigma^{x}$

$1 \sigma^{\pi}$ 
AMATHUSIIDAE (Continued)

\begin{tabular}{|c|c|c|c|}
\hline MORPHOPSIS & albertisi & $3 \sigma^{x} \sigma^{x}$ & \\
\hline & derrhyon (type) & & \\
\hline THAURIA & lathyi & $7 \sigma$ & 3 \\
\hline & intermedia & $2 \sigma^{x} \sigma^{x}$ & 1 \\
\hline & aliris & $2 \sigma^{\pi} \sigma^{7}$ & 1 \\
\hline
\end{tabular}

CLEROME \& THAUMANTIS.

DISCOPHORA

Number of specimens about.

250

TAENARIS

about

100

Forms represented by types.

about.

350

101

\section{BRASSOLIDAE}

OPSIPHANES, etc

Including :

Number of specimens about

300

fruhstorferi " $\sigma^{\top} \sigma^{\circ} \quad 1$ ?

CALIGO \& ERYPHANIS

Number of specimens about. .

250

Including :

CALIGO

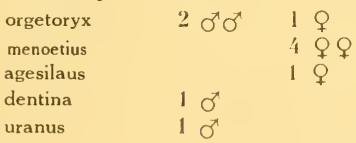

DASYOPHTHALMA.

Number of specimens about ......

DYNASTOR.

Including :

Number of specimens about....

napoleon

strix

pampanis

10

$\begin{array}{lll}3 \sigma^{\top} & 1 & 1 \\ & 1 & ?\end{array}$

MORPHIDAE

MORPHO.

Including :

Number of specimens about......

400

\begin{tabular}{|c|c|}
\hline octavia & $2 \sigma^{x}$ \\
\hline eros & 10 \\
\hline adonis & $3 \sigma^{x}$ \\
\hline phanodemus & $10^{x}$ \\
\hline$"$ & 10 \\
\hline$"$ & \\
\hline rhetenor & \\
\hline
\end{tabular}

(Ecuador)

(Peru)

(Bolivia)

Forms represented by types.

\section{SATYRIDAE (Oriental)}

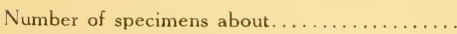


DRUSILLOPSIS doheryi $2 q$ \%

Forms represented by types about....................... 400

SATYRIDAE (Palaearctic)

Number of specimens about....................... $\quad \ldots \ldots+6.000$

Forms represented by types..................... 180

SATYRIDAE (American)

Number of specimens about........................ 450

Forms represented by types....................... 5

SATYRIDAE (African)

Number of specimens about......................... so

ERYCINIDAE (Eastern)

Number of specimens about ....................... $\quad 1.150$ including fine series of :

\section{LAXITA}

ABISARA

TAXILA

DICALLANEURA, etc.

DODONA windu $2 \sigma^{x} \quad 1$ ?

Forms represented by types.....................

ERYCINIDAE (American)

Number of specimens about.......................... 50 including many fine species.

\section{LIBYTHEINAE}

Number of specimens about........................... 200

Forms represented by types........................... 16

LYCAENIDAE (Eastern)

Number of specimens about..................... Including :

LIPHYRA brassolis

SEMANGA superba gloriosa

PORITIA very fine series of

ARHOPALA helenore
$20^{2} \sigma^{x}$

200 ธ大० क्ष

o $\quad$ \& and other fine species.

(Sikkim) 
LYCAENIDAE (Palaearctic)

Number of specimens about. ................... 1.500()

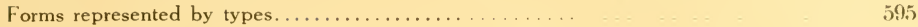

\section{HESPERIDAE}

Number of specimens about................ 20(0)

Forms represented by types................ 24)

\section{HETEROCERA}

Number of specimens about.................... (650)

Many fine species in all families.

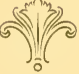





\section{Respecting the FRUHSTORFER COLLECTION}

\section{of BUTTERFLIES from IAVA Island.}

Very fine specimens, many types of Eurytella, Ergolis, Euthalia.

The LYCAENIDAE are very fine represented by about 1,300 specimens with about 150 types.

The Java Collection is represented by 3 CABINETS with walnut pannel doors, containing 120 drawers with glass bottom.

Georges Talbot.

The well known entomologist and explorer the Dr. L. Martin - Diessen, one of the best connoisseurs of the Indo-Malayan Fauna - writes in "Iris" of Nov. 30th. 1922 :

"Fruhstorfer devoted more than three years to entomological research in every part of Java that island paradise, and he declared that his stay at Java had marked the beginning of his success as a collector" - a career which, at the outset, he had found full of difficulty-and that "the years spent in that island had been the happiest of his life and the most active, the most memorable of his youth". Those who know Java, that earthly paradise, will sympathise with him. FRUHSTORFER'S KNOWLEDGE OF THE FAUNA OF JAVA HAS NEVER BEEN EXCELLED and even the Dutch who, after all, have possessed and exploited the island for more than three hundred years have never produced anyone who could be compared with him. He was the first to recognize the remarkable difference which exists between the fauna of the western part of the island where the rainfall is abundant and that of the dry eastern part, and during those years he discovered a large number of new species and varieties

The collection he formed at Java remained his most cherished possession. It was kept separate, and was never amalgamated with the rest of his Indo-Malayan collection. There was something touching in his love for all the creatures of that island which, compared with those of the same group in the other islands, he always declared to be the most beautiful, the most finely shaped and the most gorgeously coloured of all.

His enthusiasm may have been justified in many cases, but it is difficult for anyone not possessing his intimate knowledge of the Malay Archipelago to judge or to express an opinion.

It was at Java that he discovered the only known specimen of gauroides, a species of Elymnias which closely mimics an Ideopsis. No other specimen has ever been caught, either by him or by anybody else. It is possible that this specimen belonged to a species which has since become extinct and that Fruhstofer caught one of the last existing specimens; or perhaps this animal may eventually be found to be an extremely rare dimorphous female of one of the known species". 


\section{DE'TAIIAS}

of the

\section{FIRUHATORIFR .JAVA COLIACTION}

About 5,000 specimens including about 480 types.

Amongst the very interesting species in this section, we note :

\begin{tabular}{|c|c|}
\hline ZEUXIDIA PARTHAON & б \\
\hline PAPILIO LAMPSACUS & \\
\hline PAPILIO PARADOXUS & \\
\hline PAPILIO PAYENI race (2 specimens) & $\sigma$ \\
\hline PAPILIO PRILLWITZI (type, unic) & $\sigma$ \\
\hline TROIDES JUPITER & б \\
\hline CHARAXES KADENI & б \\
\hline ELYMNIAS KUNSTLERI (type) & \\
\hline ZEUXIDIA DOHRNI & \\
\hline ELYMNIAS MAHESWARA (types) & б \\
\hline ELYMNIAS LAIS (Gynandromorph) & $\sigma$ \\
\hline DODONA FRUHSTORFERI (type, unic) & \\
\hline DODONA WINDU & \\
\hline THECLA ABSOLON Hew. (2 specimens) & ఠ' \\
\hline IXIAS BALICE & \\
\hline DELIAS FRUHSTORFERI (paratypes ) & ఠ \\
\hline DELIAS BROMO & $\sigma^{x}$ \\
\hline DELIAS ALTIVAGA & 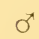 \\
\hline
\end{tabular}


DÉTAIIS

\section{Sur la Collection de Préparations}

\section{D'ORGANES GENITAUX}

\section{Des LEPIDOPTËES}

L'étude des organes génitaux des Lépidoptères, dans un but de détermination, est un procédé d'investigation récent, mais qui a donné déjà de nombreux et brillants résultats. II a permis d'établir un groupement plus logique dans les genres difficiles ; certaines espèces, voisines comme pigmentation, coupe des ailes et autres caractères morphologiques, ont pu être facilement différenciées lorsqu'on s'avisa de comparer leurs armures génitales. Des variétés, (ainsi Melitaea berisalensis, rattachées à Athalia, et qui appartient au groupe Deione), ont, avec raison, été élevées au rang d'espèces légitimes; il est donc inutile d'insister sur la valeur de ce système de recherches.

D'innombrables dissections ont amené des découvertes intéressantes, en particulier dans le genre Satyrus, celle de l'organe, que M. Fruhstorfer a nommé l'organe Jullien, lequel permet de séparer à coup sûr hermione, alcyone et les espèces voisines

La photomicrographie, donnant des images plus fidèles que celles obtenues par le dessin à la chambre claire, c'est le procédé photographique que l'on a employé de préférence, et qui a permis de publier de nombreuses Monographies de groupes dont les espèces sont si voisines par l'ensemble de leurs caractères non génitaux, qu'elles sont extrêmement difficiles à différencier.

Le Dr. I. L. Reverdin (en l'honneur duquel M. Fruhstorfer a dénommé l'organe Reverdin), a publié, dans le Bulletin de la Société Lépidopterologique de Genève, de remarquables travaux dans ce domaine.

Ces préparations demandent une grande habitude, une main experte et légère et des soins délicats.

Une collection de semblables documents forme donc un matériel d'etude de premier ordre et d'une haute valeur scientifique.

Genève, 23 Mai 1923,

(signé) JOHN JULLIEN.

Le Dr. Martin écrit, au sujet de l’emploi des préparations d'organes génitaux des Lépidoptères, dans le journal scientifique Iris, 30 Nov. 1922 :

"Fruhstorfer était, à ma connaissance, le premier qui a utilisé avec succès l'analyse anatomique d'organes génitaux des Lépidoptères pour différencier les espèces.

Mr. Fruhstorfer a réuni une collection de plus de 2.000 de ces Préparations d'organes génitaux de Lépidoptères, et presqu'autant de plaques en verre, avec les photomicrographies fort agrandies de ces préparations, représentant souvent des spécimens connus seulement en un seul exemplaire. 



\title{
PRÉPARATIONS D'ORGANES GÉNITAUX DES LÉPIDOPTËRES
}

\author{
D F'T A I LS
}

23 Boîtes spéciales, contenant environ 2,000 préparations microscopiques.

Ces préparations sont toutes sous verre et toutes déterminées. Elles représentent un matériel d'études de la plus haute valeur scientifique.

Le contenu des 23 boîtes, dites de préparations, se compose ainsi :

23 Danaidae

18 Nymphalidae

33 Catochrysops et Rapala

49 Ornithoptera et Elymnias

70 Castalius et Tarucus

76 Divers Specimens de l'Amérique.

5) Lycaenidae de l'Afrique

91 Rapala, Chliaria, Sythonidae, Deudoryx

68 Adelpha

67 Lampides

74 Satyridae, Coenonympha, Danaidae

50 Terias

47 Danaidae

76 Euthalia, Gerydinae

69 Libythea, Kallima, Eurytella

60 Papilio de l'Afrique du Sud.

10 Crenis

8 Gerydinae, Luthrodes

13 Megalura, Catonephele

5 Penthema

12 Danais

1 Nacaduba
3 Argynnis palaearct.

7 Lycaenesthes

10 Lycaenidae

7 Divers

13 Castalius

12 Lampides

6 Argynnis

15 Pieridae

9 Nymphalidae neotropiques

22 Nymphalidae trop. Orient.

12 Satyridae Pal.

11 Poritia

11 Divers

18 Euthalia

27 Adelpha

84 Lycaenesthes, Thysonotis, Luthrodes, 48 Melitaea Poritia, llerda, Catapoecilia.

73 Ageronia Crenis, Eunica africains.

89 Morphidae, Erycinidae.

80 Lycaenopsis

94 Nacaduba, Curetis.

Environ 400 Clichés Photomicrographiques représentant les agrandissements de ces Préparations d'Organes génitaux, dont :

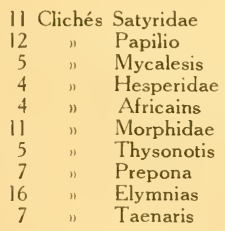

11 Clichés llerda, Tarucus

18 "Nacaduba

30 "Nymphalidae Orient.

12 "Organe Reverdin

5 ") Cacica, Lucilia

19 n Eurytelidae, Kallima, Organe

15 " Pieridae

26 "Danaidae

22 Lycaenopsis

16 " Satyridae asiatica

En outre, une collection de plus de 400 photographies toutes déterminées et tirées d'après les clichés, cités ci-dessus.

Il existe aussi une Collection de plus de 400 préparations d'ailes de papillons pour permettre l'étude approfondie de la structure. Ces préparations sous verre sont toutes déterminées et munies d'étiquettes de noms et de localités 



\section{PAge Line}

NAmE

Troides ab. auriflua form. divina

eudamidas

$\begin{array}{ll}4 & \text { " } \\ 5 & \text { " } \\ 6 & \text { " } \\ 8 & \text { " } \\ 11) & \text { " } \\ 12 & \end{array}$

Papilio kajelanus

agittatus

mosychlus

oblongomaculatus

ab. carolus

\section{albosignatus}

hector heroicus

polyphontes lingonus

aristolochiæe probus aipytos " kameitos

epycides curvatus

euchenor scribonius

$$
\text { " eutropius f. maga }
$$

gigon mangolinus

eriphonius annamiticus

xuthulinus

alcinous

neomelanides

neomelanides

ambrax decebalus

akames

ormenus

ormenulus

bridgei nobilior

neohelenus

helenus apadantus

nephelus tellonus

fuscus epibonnus

" pyrgoteles

memnon subclathratus

demetrius lenocinnus

$$
\text { n sitalkaes }
$$

rhetenor $f$. albolineata

polyctor titius

sarpedon corycus f. crocospilos luctatius

eurypylus juba

doson

arycles arycleoides

" sphinx

evemon albociliatus

doson vulso

bathycles
New Guinea

Amboina

Germ. N. G

Bazilan 


\section{PAPILIO (. Imerican)}

$$
\begin{aligned}
& \text { Papilio thoas charmadus } \\
& \text { " zagraeus chrysoxanthus } \\
& \text { " cleotas erotianus } \\
& \text { " hectorides lysirte } \\
& \text { " deicoon agordus. }
\end{aligned}
$$

\section{PIERIDAE}

Appias vacans $f$. lurida

" lyncida

$$
\text { " nera f. sufflava }
$$$$
\text { n lalage aornus }
$$$$
\text { " ada plotina }
$$$$
\text { 1) albina semiflava }
$$$$
\text { " ega plaetoria }
$$

$\begin{array}{ll}\text { Huphina timnatha } & \text { Mindanao } \\ \text { Aporia crataegi } & \text { Bazilan } \\ \text { Elodina pasarga } & \\ \text { Prioneris euclemanthe saenia } & \text { Bazilan } \\ \text { Hebomoia leucogynia } & \\ \text { " celebensis f. sublustris } & \\ \text { Catopsilia scylla scylloïdes } & \\ \text { praerubida } & \\ \text { " pomona perspicua } & \\ \text { Terias libythea avidia } & \text { Japan } \\ \text { " hecabe oeta } & \text { Kalidupa } \\ \text { " tilaha aebutia } & \text { Bazilan } \\ \text { " alitha bazilana } & \text { Bazilan } \\ \text { Pareronia phocaea erioinena } & \text { Bazgolis argolina } \\ \text { tritaea baroylia } & \text { jobaea aeboja } \\ \text { " } & \end{array}$

\section{PIERIDAE (American)}

Pereute leucodrosime

10 Archonias critias regillus

17 Catasticta bithys bithyna

18 Pieris buniae gargara

Matto Grosso 


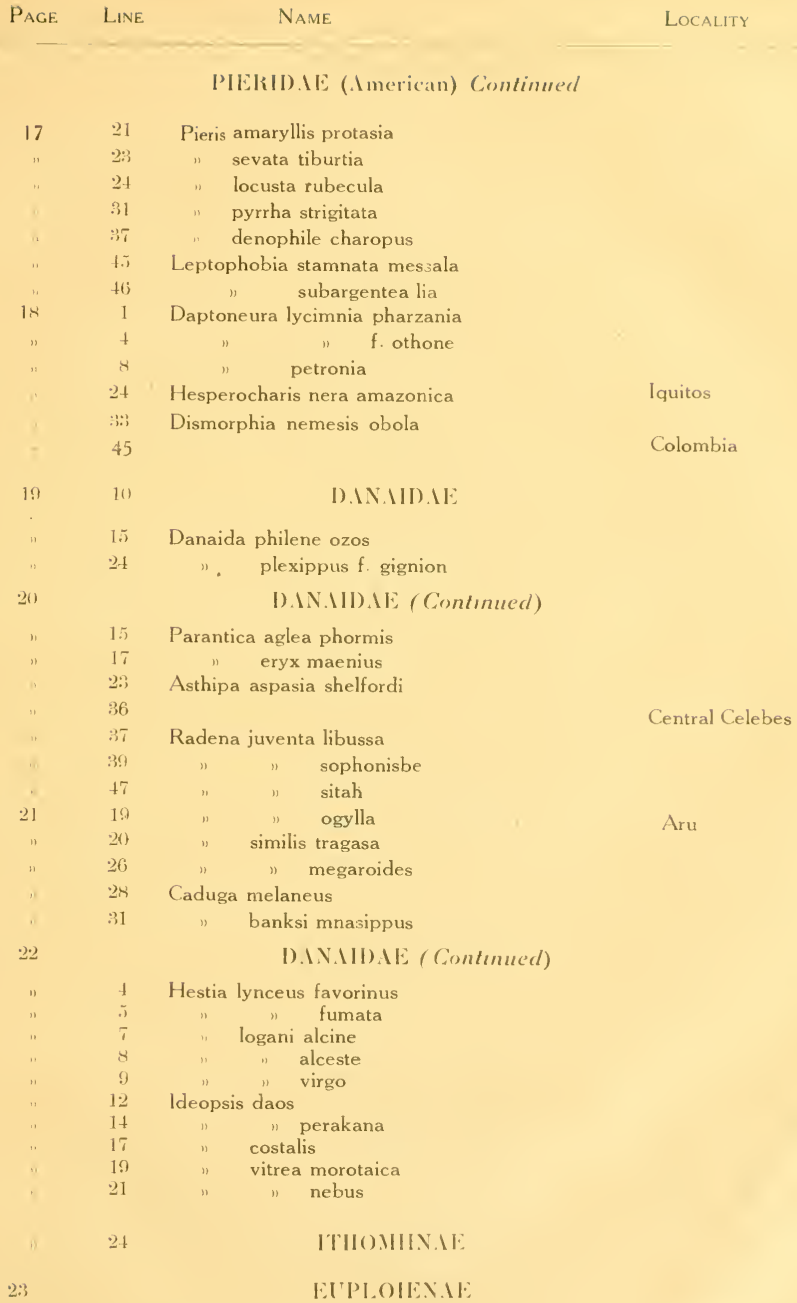




\section{EUPLOEIN.IE (Continued)}

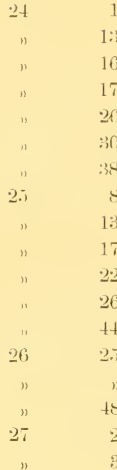

\section{Euploea alcathoae aesatia}

dione menodice

Sambawana

confusa catana

$$
\text { 1) melia }
$$

resarta tirbonia

prunosa tersatica

nemertes lycoleon

leucostictos negleyana

" virla $f$. perizonia

midamus aegumurus

aegyptus iduna

diana tombugensis

corus nikrion

hyacinthus subcongrua

pyres flammifera

cledonia paratellus

gelderi phoebaris

Bazilan

\section{NYIPIALIDAE}

Symbrenthia hippoclus batjana

$$
\text { n) }
$$

ansho

Parthenos sylvia etoga

"

n neohannoverana

\section{Euripus consimillis curinus $\mathrm{f}$. amala}

\section{Dichorragia nesimachus formosanus}

Euthalia garuda anagama

agnis modesta

monima bipunctata

f. stictica

kesava tudela

cocytina $f$ montivaga

Bazilan

adonia sumatrana

Bazilan

Bazilan

$$
\begin{aligned}
& \text { " pura } \\
& \text { armanda eutaenia } \\
& \text { anosia yenadora } \\
& \text { raja f. galana } \\
& \text { phemius seitzi } \\
& \text { aetion sositheus }
\end{aligned}
$$

Tanaecia munda f. salina

$$
\text { palguna balina }
$$

$$
\text { n stygiana }
$$

pelea crowleyi 


\section{NYMPIILAI).IE (Continued)}

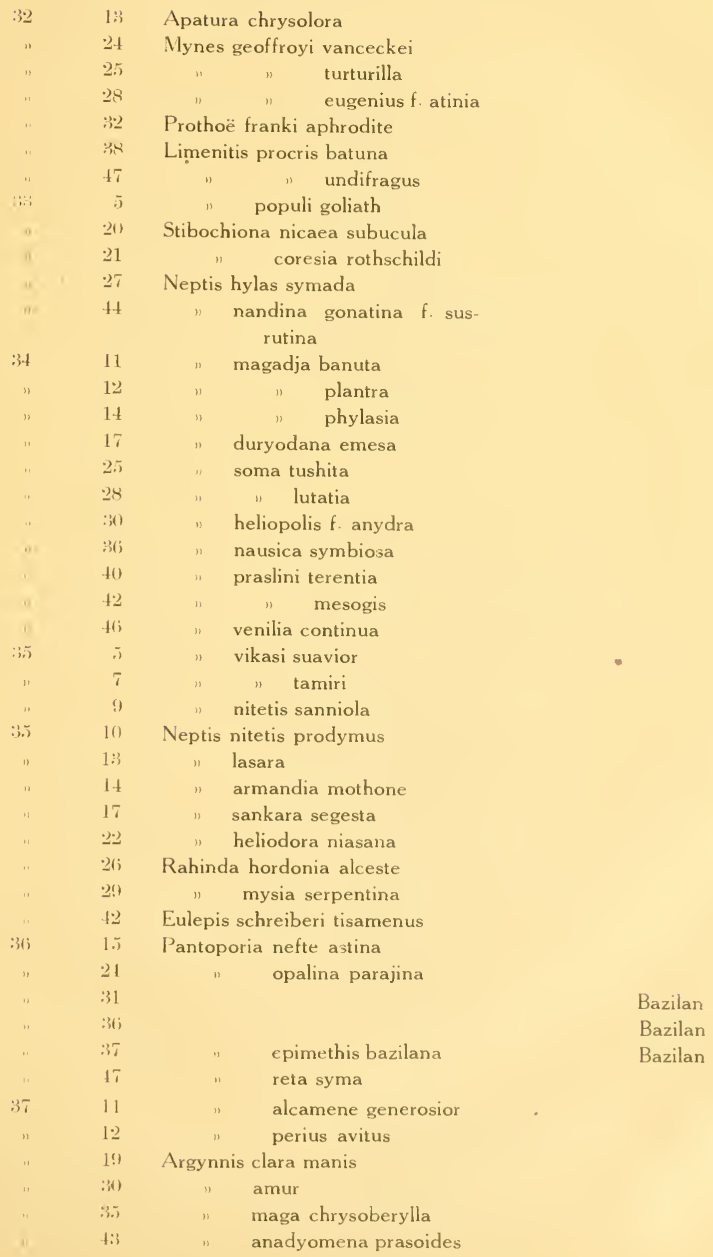


Page Line Name Locality

NYMPIIALIDAE (Continued)

38

$n$

19

29

33

34

35

36

39

36

40

41

i)

"

11 47

42

118

$$
21)
$$$$
\text { 34 }
$$$$
45
$$$$
46
$$

43

43

24

26

27

29

:3.)

4i)

45

9

29

36

37
Argynnis ruslana fregsna

hecate teriburniana

) sentia

aphirape pambotis

pales naparis

amathusia blachieri fusio

pralognana

jugnotha

euphrosyne calynota

Melitaea asteria mevania

thore hyperusia

freija calas

phoebe telona

lokris

athalia luriflua

" boris

didyma ochracea 3

$$
\text { Cupha cluentia }
$$

Cyrrochroa tyche thilina

$$
\text { calypso f. baluna }
$$

clagia clagina

aoris $\mathrm{f}$. stramentica

Cyrrochroa semiramis minos

Atella alcippe drepana

11
Pralognan

Zermath

Montelimar

Cogne

Bazilan

Bazilan 


\section{NYMPIILII).IE (Continued)}

\begin{tabular}{|c|c|c|c|c|}
\hline 44 & 36 & \multicolumn{2}{|c|}{ Vanessa kashmirensis aesis } & \\
\hline " & 40 & $"$ & canace maniliana & \\
\hline " & 4. & " & L. album samourai & \\
\hline 4.5 & 2 & \multicolumn{2}{|c|}{ Hypolimnas alimene diphridas } & \\
\hline " & 8 & $n$ & " remigia & \\
\hline$"$ & 9 & " & " bandana & \\
\hline " & 10 & " & n saturnia & \\
\hline " & 1.5 & " & antilope euvaristos & \\
\hline " & 18 & " & " stellata & \\
\hline " & 19 & " & " mela & \\
\hline " & 27 & " & pithoeka $f$ illuminata & \\
\hline 1. & $: 30$ & " & bolina f. euryxantha & \\
\hline ." & 32 & $"$ & $\begin{array}{l}\text { " lisianassa } \\
\text { f. luctuosa }\end{array}$ & \\
\hline it & 34 & " & $\begin{array}{l}\text { "philippensis } \\
\text { f. lictrix }\end{array}$ & \\
\hline n & 35 & " & $\begin{array}{l}\text { " philippensis } \\
\text { f. subucula }\end{array}$ & \\
\hline " & 42 & ") & $\begin{array}{l}\text { pandarus f. triom- } \\
\text { phans }\end{array}$ & \\
\hline ". & 44 & " & saundersi junia & \\
\hline n & $4 \bar{T}$ & " & anomala $f$ arnoldi & \\
\hline $4 t i$ & 18 & Cyrestes & acilia gades & Aroa \\
\hline$"$ & 24 & Vanessa & antiopa asopos & \\
\hline " & $3 ; 3$ & Dolesch & allia hexophtalmus ardys & \\
\hline " & 43 & " & nacar trachelus & \\
\hline$"$ & 4.) & " & bisaltide orthorgonia & \\
\hline " & 48 & " & " tenimberensis & \\
\hline 47 & 1 & " & bisaltide & \\
\hline " & 1.5 & " & " andamanensis & \\
\hline " & 22 & Yoma sa & abina vasuki $f$. sabulosa & \\
\hline " & 23 & " & " f. purpurea & \\
\hline . & 2.5 & $" a$ & alzina $f$. taurisca & \\
\hline " & 32 & Rhinopa & alpa polynice helionice & \\
\hline . & 33 & " & $\begin{array}{l}\text { " helionice } \\
\text { f. helena }\end{array}$ & \\
\hline 4 & 36 & " & " callonice & \\
\hline " & 38 & & & Bazilan \\
\hline ". & 40) & " & $\begin{array}{l}\text { tamora } \\
\mathrm{f} \text {. bellinice }\end{array}$ & \\
\hline " & 41 & " & " amrenice. & \\
\hline 4 & 42 & " & " $\quad$ eunice & \\
\hline$n$ & 43 & Kallima & inachis $f$ marmorata & \\
\hline to & 1 & $"$ & limborgi tribonia & \\
\hline$"$ & 2 & Symbren & nthia tippoclus lucianus & \\
\hline
\end{tabular}




\title{
NYMPIILII)AE (Ameriean)
}

\begin{tabular}{|c|c|c|}
\hline $4 !$ & 8 & Prepona omphale octavia \\
\hline$n$ & 11 & "dexamenes psacon \\
\hline it & \% & Adelpha calliphane \\
\hline$n$ & 8 & thessalia cesilas \\
\hline " & 20 & basiloides caelia \\
\hline$n$ & $2 \mathrm{H}$ & seriphia pione \\
\hline 5.5 & 5 & \\
\hline 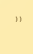 & 21 & $\begin{array}{l}\text { Catagramma codomannus paulis- } \\
\text { tanus }\end{array}$ \\
\hline$n$ & 42 & Perisama cabirnia thryoessa \\
\hline
\end{tabular}

\section{AMATHUSIIDAE}

Clerome menado sulana

\section{BRASSOLIDAE}

is

59

(ii)

13

6.)

$\begin{array}{rl}n & 13 \\ n & 15 \\ 37 & 37 \\ 66 & 12 \\ n & 23 \\ 67 & 18 \\ 69 & 36\end{array}$

70

Caligo oileus philinos

Catoblebia cyparissa

\section{SAT YRIDAE}

\section{Melanargia galathea convena}

\author{
INI)(OMALAYAN
}

Mycalesis discobolus

$$
\begin{aligned}
& \text { " haasei unipupillata } \\
& \text { Ptychandra lorquini caerulans } \\
& \text { Lethe neoclides }
\end{aligned}
$$

" arete vaya

Hypocysta busiris

\section{ELYUNIINAE}
Elymnias panthera alfredi
Elymnias hewitsoni meliophila " vitellia $f$ basinus 


\begin{tabular}{|c|c|c|}
\hline PAGE & LINE & NAME \\
\hline$x: 3$ & & LYCAENIDAE \\
\hline$n$ & i & Zephyrus japonica f. amethystina \\
\hline$"$ & $\tau$ & $\begin{array}{l}\text { Heliophyrus epicles indicus } \\
\text { f. rufonalates }\end{array}$ \\
\hline 8.5 & 35 & Castalius roxus satrannus \\
\hline 47 & 12 & Logania subviolascens silarus \\
\hline$n$ & 18 & I" marmorata stenosa \\
\hline$"$ & 49 & Hypolycaena erylus pupienus \\
\hline Si & 1 & " pupienus siphax \\
\hline$n$ & 17 & Loxura atymnus furonius \\
\hline - & 18 & $" \quad$ " leminius \\
\hline
\end{tabular}

\section{SECOND LIST OF TYPES}

Delias crithoë villia.

" hyparete peirene f. maenia

$$
\text { decombesif. leucogaea }
$$

$$
\begin{array}{lll}
\text { " " } & \text { eranthos } \\
\text { " } & \text { f. auriga }
\end{array}
$$

lxias reinhardti lombokiana

4.) Terias andersoni undana

$$
\text { NTMPIILII).IE }
$$

$$
\text { Cynthia erota javana }
$$

Neptis yerburyi dumicola

ab. gedeana

$$
\text { parthica } f \text {. somula }
$$

Dichorrhagia nesimachus mannus

4.2 Prothoë australis menodora

$$
\text { BR.ISS()LID.IE }
$$

Opsiphanes cassina munatius

$$
\text { S.ITYRIIOAE P.IL.AE.IRC.TIC: }
$$

2:3 Coenonympha gallacia

$$
\text { ERICINIIIAE }
$$

:2. Dodona adonira argentea

$$
\begin{aligned}
\text { Omissions : } & \text { EUPLOEINAE } \\
\text { Page 27 } & \text { Line 24 } \\
& \text { Key } \\
& \text { Obi } \\
& \text { Fergussor }{ }_{1} \\
& \text { Dutch N Guinea }
\end{aligned}
$$

Euploea alecto nymphas

1) zodica

barsine

" fidena 
1 mpression de Luxe

J. Gastaud. Nice - Teléph 39.74 


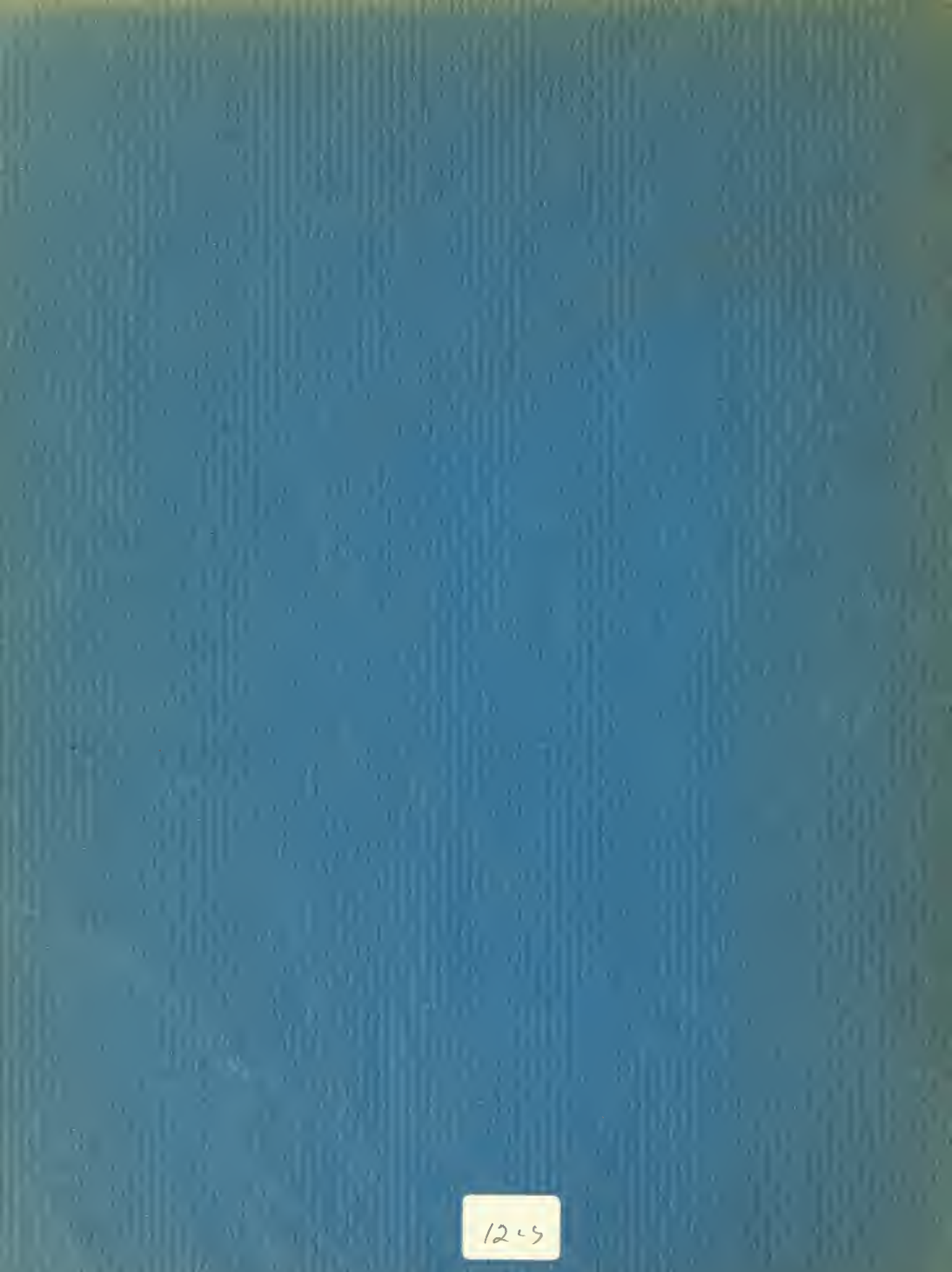





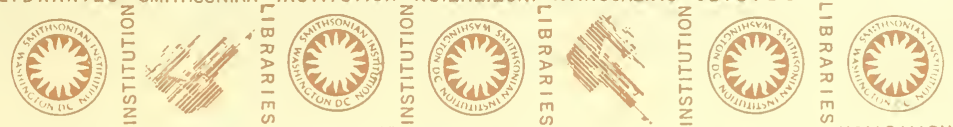

NOILOIIISNI NHINOSHLIWS S $318 \forall 8817$ LIBRARIES
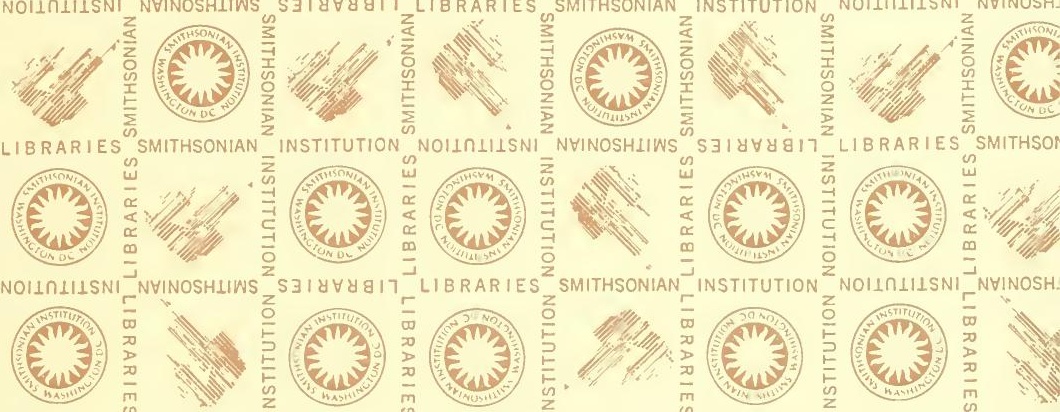

I BRARIES SMITHSONIAN INSTITUTION NOIINIIISNI
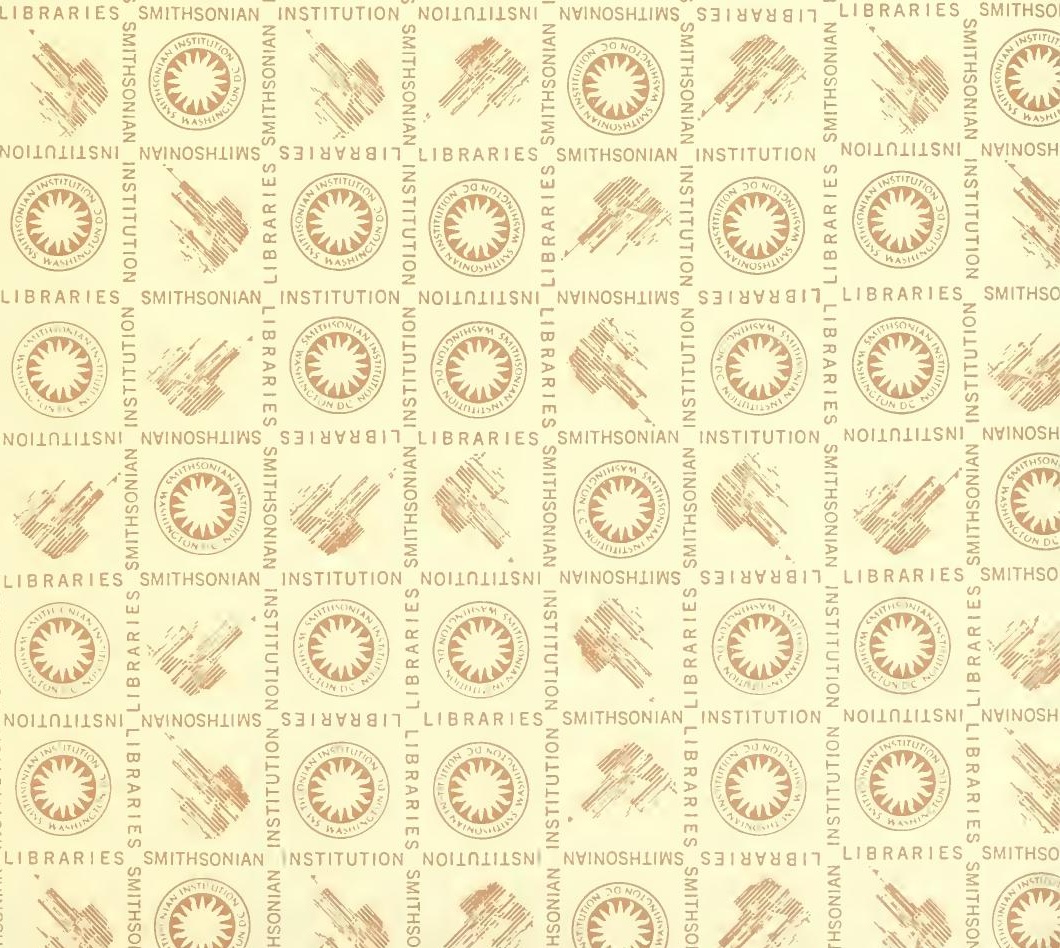

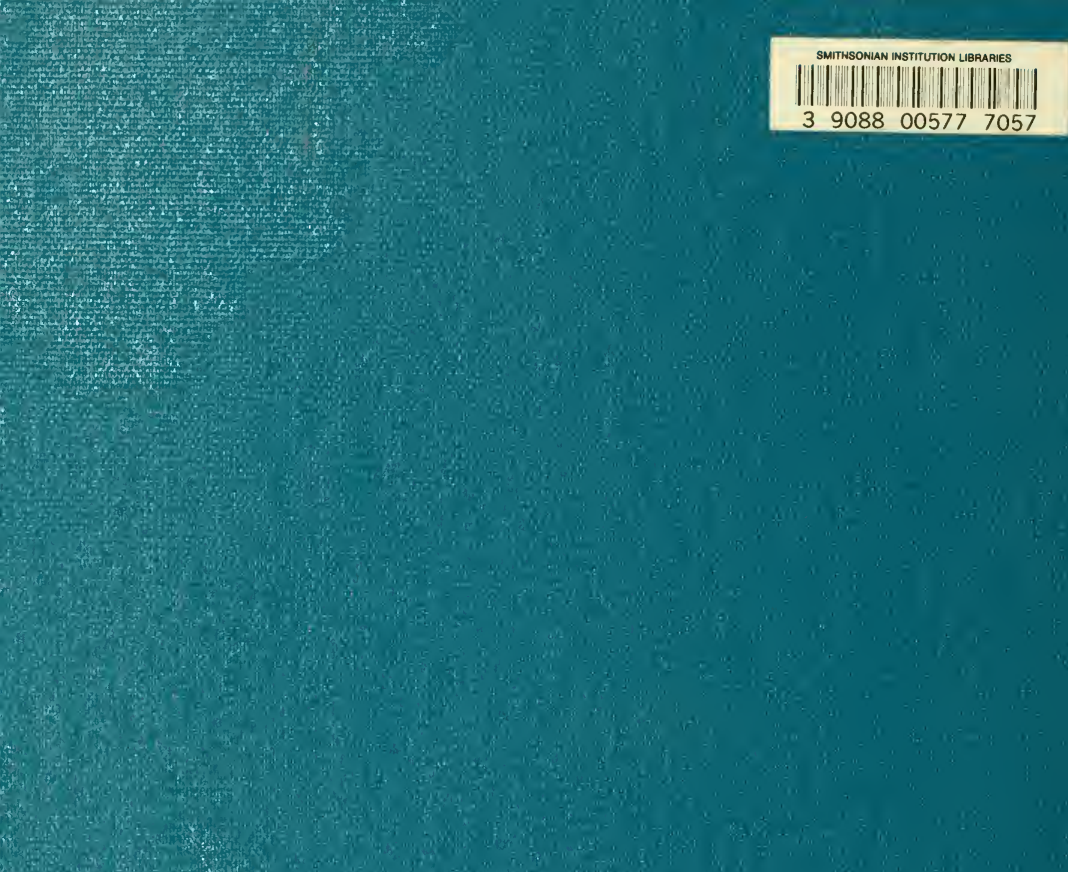